\title{
CONVERGENCE ANALYSIS OF A FAMILY OF ELLAM SCHEMES FOR A FULLY COUPLED MODEL OF MISCIBLE DISPLACEMENT IN POROUS MEDIA
}

\author{
HANZ MARTIN CHENG, JÉRÔME DRONIOU, AND KIM-NGAN LE
}

\begin{abstract}
We analyse the convergence of numerical schemes in the GDMELLAM (Gradient Discretisation Method-Eulerian Lagrangian Localised Adjoint Method) framework for a strongly coupled elliptic-parabolic PDE which models miscible displacement in porous media. These schemes include, but are not limited to Mixed Finite Element-ELLAM and Hybrid Mimetic MixedELLAM schemes. A complete convergence analysis is presented on the coupled model, using only weak regularity assumptions on the solution (which are satisfied in practical applications), and not relying on $L^{\infty}$ bounds (which are impossible to ensure at the discrete level given the anisotropic diffusion tensors and the general grids used in applications).
\end{abstract}

\section{Model and ASSUmptions}

We consider the following coupled system of partial differential equations, modelling the miscible displacement of one fluid by another in a porous medium:

$$
\begin{gathered}
\operatorname{div} \mathbf{u}=q^{+}-q^{-} \quad \text { on } Q:=\Omega \times(0, T) \\
\mathbf{u}=-\frac{\mathbf{K}}{\mu(c)} \nabla p \quad \text { on } Q \\
\phi \frac{\partial c}{\partial t}+\operatorname{div}(\mathbf{u} c-\mathbf{D}(\mathbf{x}, \mathbf{u}) \nabla c)+q^{-} c=q^{+} \quad \text { on } Q
\end{gathered}
$$

with unknowns $p(\boldsymbol{x}, t), \mathbf{u}(\boldsymbol{x}, t)$, and $c(\boldsymbol{x}, t)$ which denote the pressure of the mixture, the Darcy velocity, and the concentration of the injected solvent, respectively. The functions $q^{+}$and $q^{-}$represent the injection and production wells respectively, and $\mathbf{D}(\mathbf{x}, \mathbf{u})$ denotes the diffusion-dispersion tensor

$$
\mathbf{D}(\mathbf{x}, \mathbf{u})=\phi(\mathbf{x})\left[d_{m} \mathbf{I}+d_{l}|\mathbf{u}| E(\mathbf{u})+d_{t}|\mathbf{u}|(\mathbf{I}-E(\mathbf{u}))\right] \text { with } E(\mathbf{u})=\left(\frac{u_{i} u_{j}}{|\mathbf{u}|^{2}}\right)_{i, j} .
$$

Here, $d_{m}>0$ is the molecular diffusion coefficient, $d_{l}>0$ and $d_{t}>0$ are the longitudinal and transverse dispersion coefficients respectively, and $E(\mathbf{u})$ is the projection matrix along the direction of $\mathbf{u}$. Also, $\mathbf{K}$ is the symmetric, bounded uniformly coercive diffusion tensor, and $\mu(c)=\mu(0)\left[(1-c)+M^{1 / 4} c\right]^{-4}$ is the viscosity of the fluid mixture, where $M=\mu(0) / \mu(1)$ is the mobility ratio of the

Date: September 11, 2018.

2010 Mathematics Subject Classification. 65M08, 65M12, 65M25, 65M60, 76S05 .

Key words and phrases. flows in porous media, gradient discretisation method, gradient schemes, Eulerian Lagrangian Localised Adjoint Method, coupled system, convergence analysis. 
two fluids. As usually considered in numerical tests, we take no-flow boundary conditions:

$$
\mathbf{u} \cdot \mathbf{n}=(\mathbf{D} \nabla c) \cdot \mathbf{n}=0 \text { on } \partial \Omega \times(0, T) .
$$

The concentration equation is completed by an initial condition, and the pressure equation by an average condition:

$$
c(\boldsymbol{x}, 0)=c_{\text {ini }} \text { for all } \boldsymbol{x} \in \Omega, \int_{\Omega} p(\boldsymbol{x}, t) d \boldsymbol{x}=0 \text { for all } t \in(0, T) .
$$

Problem (1) is used in enhanced oil recovery. Exact solutions of this model are usually inaccessible, especially with data as encountered in applications; thus the design and convergence analysis of numerical schemes for (1) is therefore of particular importance. The main purpose of this work is to provide a GDM-ELLAM (Gradient Discretisation Method-Eulerian Lagrangian Localised Adjoint Method) framework for model (1) and to establish convergence of numerical schemes that fall under this framework. Some of the schemes covered by this framework are the Mixed Finite Element-ELLAM and Hybrid Mimetic Mixed-ELLAM schemes. An overview of studies and analysis involving ELLAM schemes is presented in [30]. Convergence analysis was performed for MFEM-ELLAM schemes (or similar) in $[5,31]$. We note here that [5] only considers the concentration equation (1b) (assuming that $\mathbf{u}$ is given), whereas [31] provides error estimates for the complete coupled model (1). However, these analysis were carried out under restrictive regularity assumptions on the porosity $\phi$ and on the solution $(p, \mathbf{u}, c)$ to the model; in particular, the minimal assumptions in [31] are $c \in H^{1}\left(0, T ; H^{2}(\Omega)\right) \cap L^{\infty}\left(0, T ; W^{2, r}(\Omega)\right.$ ) (for $r>2)$ and $\mathbf{u} \in W^{1, \infty}(\Omega \times(0, T))$, and [5] supposes that $c, \mathbf{D} \nabla c \in C^{1}\left(0, T ; H^{1}(\Omega)\right)$ and $\phi, \mathbf{u} \in W^{1, \infty}(\Omega \times(0, T))$. However, in reservoir modeling, transitions between different rock layers are usually discontinuous; thus, the permeability may vary rapidly over several orders of magnitude, with local variations in the range of $1 \mathrm{mD}$ to $10 \mathrm{D}$, where D is the Darcy unit [28]. Due to this discontinuity of $\mathbf{K}$, the solutions to (1) cannot expect to satisfy the regularity conditions stated above. Actually, all reported numerical tests $[8,9,11,32]$ seem to have been on tests cases for which such regularity of the data and/or the solutions do not hold.

More recent developments of ELLAM techniques involve Volume Corrected Characteristic Mixed Methods (VCCMM), which are, in essence, ELLAM schemes with volume adjustment to achieve local mass conservation. Convergence analysis, as well as stability, monotonicity, maximum and minimum principles for these schemes have been studied in $[3,4]$. However, these studies only consider a single pure advection model (that is, (1b) with $\mathbf{D}=0$ ), and assume the regularity $\mathbf{u} \in C^{1}(\Omega \times(0, T)$ ), which, as explained above, is not expected in applications. Without accounting for diffusion, the maximum principle is accessible, and thus the analysis strongly benefits from the resulting $L^{\infty}$ bounds on the approximate solution. On the contrary, in the presence of anisotropic heterogeneous diffusion $\mathbf{K}$ and $\mathbf{D}(\mathbf{u})$, and on grids as encountered in applications, constructing schemes that satisfy the maximum principle is extremely difficult - to this day, only nonlinear schemes are known to preserve the maximum principle in general, and even these do not necessarily have nice coercivity features [14].

As a matter of fact, the convergence analysis of numerical approximations of (1) under weak regularity assumptions has recently received an increasing interest; see, 
e.g., $[8,10]$ for finite volume methods and $[25,29]$ for discontinuous Galerkin methods. It therefore seems natural to consider doing such an analysis for characteristicbased discretisation of the advection term. This leaves open the choice of particular discretisations of the diffusion terms in the model. Instead of selecting one particular discretisation of these terms, we work inside a framework that enables a simultaneous analysis of various such discretisations.

The Gradient Discretisation Method is a generic framework to discretise diffusion equations [16]. It consists in replacing, in the weak formulation of the equation, the continuous space and functions/gradients by a discrete space and reconstructions of functions/gradients. This space together with the reconstruction operators are called a gradient discretisation (GD). The convergence of the resulting scheme is ensured under a few properties ( 3 or 4 , depending on the non-linearities in the model) on the GDs. The efficiency of the GDM is found in its flexibility: various choices of GDs lead to various classical methods (conforming and non-conforming finite elements, finite volumes, etc.), which means that the analysis carried out in the GDM directly applies to all these methods at once.

The main contributions of this work are

- Presentation of a GDM-ELLAM framework for the complete coupled model (1), which covers a variety of discretisations of the diffusion terms.

- Convergence analysis that only relies on energy estimates based on coercivity but not maximum principle, and is therefore adapted to anisotropic heterogeneous diffusion on generic grids as encountered in applications.

- Analysis carried out under weak regularity assumptions on the data, as seen in previously reported numerical tests on various schemes for (1).

The paper is made up of two main components, a conclusion and an appendix. The first main component (Sections 2 to 4) focuses on the presentation of the GDM-ELLAM, the main convergence result, and numerical schemes that fall into this framework; whereas the second component (Sections 5 to 7 ) establishes properties on the flows, a priori estimates on the solution to the scheme, and prove its convergence by using compactness techniques. The conclusion (Section 8) recalls the main elements of the paper, and the appendix (Section 9) contains a few technical compactness results.

We start by presenting the weak formulation of the model (1). This is followed by Section 2, which gives a short overview of the gradient discretisation method, together with some standard properties which ensure the convergence of the gradient schemes. Section 3 then presents the GDM-ELLAM for the model (1), followed by the main results: existence and uniqueness of the solution to the scheme, and its convergence to the weak solution of (1) under weak regularity assumptions. Section 4 then gives some of the numerical schemes that are covered by the GDM-ELLAM framework, together with proofs on why they satisfy the regularity assumptions.

Since ELLAM schemes are based on characteristic methods, we need to solve characteristics along which the solution flows. The properties of the flow (described by the characteristics), together with some estimates that come with it, are presented in Section 5. These properties are not trivial to establish due to the weak regularity assumptions. A priori estimates are then obtained in Section 6, which lead us to compactness arguments that will help establish the proof of convergence. Finally, we prove our convergence result in Section 7. The ELLAM discretisation 
of the advection term makes the energy estimates and the convergence analysis of the corresponding terms rather tricky. The fine results from Sections 5 and 6 are instrumental to obtain the major estimates and the proper convergence of the advection term. We also note that, at the core of our convergence analysis lies some generic compactness results of [16], which are flexible enough to be used even outside a purely GDM framework (as in the GDM-ELLAM framework here).

Throughout the article we assume the following properties, satisfied by $\mathbf{D}, \mathbf{K}$ and $\mu$ previously described.

$$
\begin{aligned}
& c_{\text {ini }} \in L^{\infty}(\Omega) \text { and } q^{+}, q^{-} \in L^{\infty}(\Omega \times(0, T)) \text { with }\left|q^{+}\right| \leq M_{q^{+}},\left|q^{-}\right| \leq M_{q^{-}} \\
& \phi \text { is piecewise smooth on a mesh, and there exists } \phi_{*}, \phi^{*}>0 \text { such that } \\
& \phi_{*} \leq \phi \leq \phi^{*} \text { on } \Omega \text {. } \\
& A=\mathbf{K} / \mu \text { is Carathéodory and there exists } \alpha_{A} \text { and } \Lambda_{A} \text { s.t. for a.e. } \boldsymbol{x} \in \Omega, \\
& \forall(s, \xi) \in \mathbb{R} \times \mathbb{R}^{d}: A(\boldsymbol{x}, s) \xi \cdot \xi \geq \alpha_{A}|\xi|^{2} \text { and }|A(\boldsymbol{x}, s)| \leq \Lambda_{A} \text {. } \\
& \mathbf{D} \text { is Carathéodory and there exists } \alpha_{\mathbf{D}} \text { and } \Lambda_{\mathbf{D}} \text { s.t. for a.e. } \boldsymbol{x} \in \Omega \\
& \forall \xi, \zeta \in \mathbb{R}^{d}: \mathbf{D}(\boldsymbol{x}, \zeta) \xi \cdot \xi \geq \alpha_{\mathbf{D}}(1+|\zeta|)|\xi|^{2} \text { and }|\mathbf{D}(\boldsymbol{x}, \zeta)| \leq \Lambda_{\mathbf{D}}(1+|\zeta|) .
\end{aligned}
$$

Here, "Carathéodory" means measurable with respect to $\boldsymbol{x}$ and continuous with respect to the other variables. In $(2 \mathrm{~b})$ as well as $(\mathbf{A 4})$ below, "mesh" is to be understood in the simplest intuitive way: a partition of $\Omega$ into polygonal (in 2D) or polyhedral (in 3D) sets. Under these assumptions, we consider the following standard notion of weak solution to (1) (see, e.g., [24]).

Definition 1.1 (Weak solution to the miscible displacement model). A couple $(p, c)$ is a weak solution of (1) if

$$
\begin{aligned}
& p \in L^{\infty}\left(0, T ; H^{1}(\Omega)\right), \int_{\Omega} p(\boldsymbol{x}, t) d \boldsymbol{x}=0 \text { for a.e. } t \in(0, T), \text { and } \\
& \int_{0}^{T} \int_{\Omega} \frac{\mathbf{K}(\boldsymbol{x})}{\mu(c(\boldsymbol{x}, t))} \nabla p(\boldsymbol{x}, t) \cdot \nabla \psi(\boldsymbol{x}, t) d \boldsymbol{x} \mathrm{d} t \\
& \quad=\int_{0}^{T} \int_{\Omega}\left(q^{+}(\boldsymbol{x}, t)-q^{-}(\boldsymbol{x}, t)\right) \psi(\boldsymbol{x}, t) d \boldsymbol{x} d t, \quad \forall \psi \in C^{\infty}(\bar{\Omega} \times[0, T]),
\end{aligned}
$$

and, setting $\mathbf{u}(\boldsymbol{x}, t)=-\frac{\mathbf{K}(\boldsymbol{x})}{\mu(c(\boldsymbol{x}, t))} \nabla p(\boldsymbol{x}, t)$,

$$
\begin{aligned}
c & \in L^{2}\left(0, T ; H^{1}(\Omega)\right),(1+|\mathbf{u}|)^{1 / 2} \nabla c \in L^{2}(\Omega \times(0, T))^{d}, \\
- & \int_{\Omega} \phi(\boldsymbol{x}) c_{\mathrm{ini}}(\boldsymbol{x}) \varphi(\boldsymbol{x}, 0) d \boldsymbol{x}-\int_{0}^{T} \int_{\Omega} \phi(\boldsymbol{x}) c(\boldsymbol{x}, t) \frac{\partial \varphi}{\partial t}(\boldsymbol{x}, t) d \boldsymbol{x} d t \\
& +\int_{0}^{T} \int_{\Omega} \mathbf{D}(\boldsymbol{x}, \mathbf{u}(\boldsymbol{x}, t)) \nabla c(\boldsymbol{x}, t) \cdot \nabla \varphi(\boldsymbol{x}, t) d \boldsymbol{x} d t \\
& -\int_{0}^{T} \int_{\Omega} c(\boldsymbol{x}, t) \mathbf{u}(\boldsymbol{x}, t) \cdot \nabla \varphi(\boldsymbol{x}, t) d \boldsymbol{x} d t+\int_{0}^{T} \int_{\Omega} q^{-}(\boldsymbol{x}, t) c(\boldsymbol{x}, t) \varphi(\boldsymbol{x}, t) d \boldsymbol{x} d t \\
& =\int_{0}^{T} \int_{\Omega} q^{+}(\boldsymbol{x}, t) \varphi(\boldsymbol{x}, t) d \boldsymbol{x} d t, \quad \forall \varphi \in C_{c}^{\infty}(\bar{\Omega} \times[0, T)) .
\end{aligned}
$$


Remark 1.2 (Injection concentration and gravity). The model (1) assumes an injection concentration of 1 and neglects the gravity effects. A generic injection concentration $\widehat{c}$ could be considered upon the trivial modification $q^{+} \leadsto \widehat{c} q^{+}$in (1b). To include gravity effect, we would have to set $\mathbf{u}=-\frac{\mathbf{K}}{\mu(c)}(\nabla p-\rho(c) \mathbf{g})$ (with $\rho$ a continuous function). The analysis we conduct thereafter can easily be adapted to both changes.

\section{Brief PRESENTATION OF THE GRADIENT DisCRETISATION METHOD}

The gradient discretisation method (GDM) is a discretisation method for diffusion equations which consists in replacing, in the weak formulation of the PDE, the continuous space and time operators by discrete counterparts [16]. These discrete elements are given by what is a called a gradient discretisation (GD). The convergence of the resulting schemes (called gradient schemes (GS)) can be established under a few assumptions on the gradient discretisations. We give here a brief presentation of GDs and the standard properties that ensure the convergence of the corresponding GSs for standard elliptic and parabolic PDEs. In the rest of the paper, the notations $L^{p}(X)$ and $L^{p}\left(0, T ; L^{q}(X)\right)$ are sometimes also used in lieu of $L^{p}(X)^{d}$ and $L^{p}\left(0, T ; L^{q}(X)\right)^{d}$.

Definition 2.1 (Space and space-time gradient discretisations). A space gradient discretisation for no-flow boundary conditions is $\mathcal{D}=\left(X_{\mathcal{D}}, \Pi_{\mathcal{D}}, \nabla_{\mathcal{D}}\right)$, where

- the set of discrete unknowns $X_{\mathcal{D}}$ is a finite dimensional vector space on $\mathbb{R}$

- the function reconstruction $\Pi_{\mathcal{D}}: X_{\mathcal{D}} \rightarrow L^{\infty}(\Omega)$ is linear

- the gradient reconstruction $\nabla_{\mathcal{D}}: X_{\mathcal{D}} \rightarrow L^{\infty}(\Omega)^{d}$ is linear.

The operators $\Pi_{\mathcal{D}}$ and $\nabla_{\mathcal{D}}$ must be chosen so that

$$
\|v\|_{\mathcal{D}}:=\left(\left\|\nabla_{\mathcal{D}} v\right\|_{L^{2}(\Omega)}^{2}+\left|\int_{\Omega} \Pi_{\mathcal{D}} v(\boldsymbol{x}) d \boldsymbol{x}\right|^{2}\right)^{\frac{1}{2}}
$$

is a norm on $X_{\mathcal{D}}$.

A space-time gradient discretisation is $\mathcal{D}^{T}=\left(\mathcal{D}, \mathcal{I}_{\mathcal{D}},\left(t^{(n)}\right)_{n=0, \ldots, N}\right)$ such that $\mathcal{D}$ is a space $G D, 0=t^{(0)}<\cdots<t^{(N)}=T$ are time steps, and $\mathcal{I}_{\mathcal{D}}: L^{\infty}(\Omega) \rightarrow X_{\mathcal{D}}$ is an operator used to interpolate initial conditions onto the unknowns.

Considering for example (3) and replacing the space $H^{1}(\Omega)$ with $X_{\mathcal{D}}$, the functions by reconstructions using $\Pi_{\mathcal{D}}$ and the gradients by reconstructions $\nabla_{\mathcal{D}}$, we obtain the corresponding gradient scheme (6). The simplest example of a GD can be described by considering $\mathbb{P}_{1}$ finite elements on a simplicial mesh. A vector $v \in X_{\mathcal{D}}$ is made of vertex values $\left(v_{\boldsymbol{s}}\right)_{\boldsymbol{s}}$ vertex of the mesh, $\Pi_{\mathcal{D}} v$ is the continuous, piecewise linear reconstruction from these values, and $\nabla_{\mathcal{D}} v$ is the standard gradient of this reconstruction. Other examples of GDs are given in Section 4.

The accuracy of a GD and convergence properties of the resulting GS are measured through three parameters, that respectively correspond to a discrete PoincaréWirtinger constant, an interpolation error, and a measure of defect of conformity (error in a discrete Stokes formula):

$$
\begin{aligned}
& C_{\mathcal{D}}=\max _{v \in X_{\mathcal{D}}} \frac{\left\|\Pi_{\mathcal{D}} v\right\|_{L^{2}(\Omega)}}{\|v\|_{\mathcal{D}}}, \\
& \forall \varphi \in H^{1}(\Omega), S_{\mathcal{D}}(\varphi)=\min _{v \in X_{\mathcal{D}}}\left(\left\|\Pi_{\mathcal{D}} v-\varphi\right\|_{L^{2}(\Omega)}+\left\|\nabla_{\mathcal{D}} v-\nabla \varphi\right\|_{L^{2}(\Omega)}\right),
\end{aligned}
$$




$$
\forall \boldsymbol{\phi} \in H_{\text {div }}(\Omega), W_{\mathcal{D}}(\phi)=\max _{v \in X_{\mathcal{D}} \backslash\{0\}} \frac{\left|\int_{\Omega}\left(\nabla_{\mathcal{D}} v(\boldsymbol{x}) \cdot \boldsymbol{\phi}(\boldsymbol{x})+\Pi_{\mathcal{D}} v(\boldsymbol{x}) \operatorname{div} \phi(\boldsymbol{x})\right) d \boldsymbol{x}\right|}{\|v\|_{\mathcal{D}}} .
$$

Definition 2.2 (Properties of GDs). A sequence $\left(\mathcal{D}_{m}\right)_{m \in \mathbb{N}}$ of space gradient discretisations is

- coercive if there exists $C_{p} \in \mathbb{R}_{+}$such that $C_{\mathcal{D}_{m}} \leq C_{p}$ for all $m \in \mathbb{N}$,

- GD-consistent if, for all $\varphi \in H^{1}(\Omega), S_{\mathcal{D}_{m}}(\varphi) \rightarrow 0$ as $m \rightarrow \infty$,

- limit-conforming if, for all $\phi \in H_{\mathrm{div}}(\Omega), W_{\mathcal{D}_{m}}(\phi) \rightarrow 0$ as $m \rightarrow \infty$,

- compact if for any sequence $v_{m} \in X_{\mathcal{D}_{m}}$ such that $\left(\left\|v_{m}\right\|_{\mathcal{D}_{m}}\right)_{m \in \mathbb{N}}$ is bounded, the sequence $\left(\Pi_{\mathcal{D}_{m}} v_{m}\right)_{m \in \mathbb{N}}$ is relatively compact in $L^{2}(\Omega)$.

A sequence of space-time gradient discretisations $\left(\mathcal{D}_{m}^{T}\right)_{m \in \mathbb{N}}$ is coercive, limit-conforming or compact if its underlying sequence of space gradient discretisations satisfy the corresponding property. Finally, $\left(\mathcal{D}_{m}^{T}\right)_{m \in \mathbb{N}}$ is GD-consistent if the underlying sequence of spatial GDs is GD-consistent and if

- with $\delta t_{m}^{\left(n+\frac{1}{2}\right)}=t_{m}^{(n+1)}-t_{m}^{(n)}, \max _{n=0, \ldots, N_{m}-1} \delta t_{m}^{\left(n+\frac{1}{2}\right)} \rightarrow 0$ as $m \rightarrow \infty$,

- for all $\varphi \in L^{\infty}(\Omega),\left(\Pi_{\mathcal{D}_{m}} \mathcal{I}_{\mathcal{D}_{m}} \varphi\right)_{m \in \mathbb{N}}$ is bounded in $L^{\infty}(\Omega)$ and converges to $\varphi$ in $L^{2}(\Omega)$ as $m \rightarrow \infty$.

Remark 2.3. Actually, the limit-conformity or compactness of a sequence of space GDs implies its coercivity. The latter is however explicitly mentioned as a bound on $C_{\mathcal{D}_{m}}$ is useful throughout the analysis.

In the $G D M$, the interpolant $\mathcal{I}_{\mathcal{D}}$ is usually defined on $L^{2}(\Omega)$; in the context of Problem (1), the initial condition is always assumed to be bounded and it is therefore natural to only consider interpolants of initial conditions in $L^{\infty}(\Omega)$.

If $\mathcal{D}$ is a space GD, $0=t^{(0)}<\cdots<t^{(N)}=T$ are time steps and $z=$ $\left(z^{(n)}\right)_{n=0, \ldots, N} \in X_{\mathcal{D}}^{N+1}$, we define the space-time reconstructions $\Pi_{\mathcal{D}} z \in L^{\infty}(\Omega \times$ $(0, T)), \widetilde{\Pi}_{\mathcal{D}} z \in L^{\infty}(\Omega \times(0, T))$ and $\nabla_{\mathcal{D}} z \in L^{\infty}(\Omega \times(0, T))^{d}$ by

$$
\begin{aligned}
& \forall n=0, \ldots, N-1, \forall t \in\left(t^{(n)}, t^{(n+1)}\right], \text { for a.e. } \boldsymbol{x} \in \Omega, \\
& \Pi_{\mathcal{D}} z(\boldsymbol{x}, t)=\Pi_{\mathcal{D}} z^{(n+1)}(\boldsymbol{x}), \widetilde{\Pi}_{\mathcal{D}} z(\boldsymbol{x}, t)=\Pi_{\mathcal{D}} z^{(n)}(\boldsymbol{x}) \\
& \text { and } \nabla_{\mathcal{D}} z(\boldsymbol{x}, t)=\nabla_{\mathcal{D}} z^{(n+1)}(\boldsymbol{x}) .
\end{aligned}
$$

\section{GDM-ELLAM SCHEME AND MAIN RESULT}

The diffusion terms in (1a) and (1b) are discretised by the gradient discretisation method. This enables us to carry out a unified convergence analysis for many different numerical discretisations of these diffusion terms. There are grounds for considering possibly different GDs for each equation in (1) (see e.g. Section 4.1.1). We therefore take a space gradient discretisation $\mathcal{P}=\left(X_{\mathcal{P}}, \Pi_{\mathcal{P}}, \nabla_{\mathcal{P}}\right)$ for the pressure, and a space-time gradient discretisation $\mathcal{C}^{T}=\left(\mathcal{C}, \mathcal{I}_{\mathcal{D}},\left(t^{(n)}\right)_{n=0, \ldots, N}\right)$ for the concentration.

From here onwards, the variables $\boldsymbol{x}$ and $t$ may be dropped in the integrals when there are no risks of confusion.

Definition 3.1 (GDM-ELLAM scheme). The gradient scheme for (1) reads as: find $\left(p^{(n)}\right)_{n=1, \ldots, N} \in X_{\mathcal{P}}^{N}$ and $\left(c^{(n)}\right)_{n=0, \ldots, N} \in X_{\mathcal{C}}^{N+1}$ such that $c^{(0)}=\mathcal{I}_{\mathcal{C}} c_{\text {ini }}$ and, for all $n=0, \ldots, N-1$, 
i) $p^{(n+1)}$ solves

$$
\begin{aligned}
& \int_{\Omega} \Pi_{\mathcal{P}} p^{(n+1)}=0 \text { and } \\
& \int_{\Omega} A\left(\boldsymbol{x}, \Pi_{\mathcal{C}} c^{(n)}\right) \nabla_{\mathcal{P}} p^{(n+1)} \cdot \nabla_{\mathcal{P}} z=\int_{\Omega}\left(q_{n}^{+}-q_{n}^{-}\right) \Pi_{\mathcal{P}} z, \quad \forall z \in X_{\mathcal{P}}
\end{aligned}
$$

where $q_{n}^{ \pm}(\cdot)=\frac{1}{\delta t^{\left(n+\frac{1}{2}\right)}} \int_{t^{(n)}}^{t^{(n+1)}} q^{ \pm}(\cdot, s) d s$ (or, alternatively, $q_{n}^{ \pm}=q^{ \pm}\left(t^{(n)}\right)$ if $q^{ \pm}$are continuous in time).

ii) A Darcy velocity $\mathbf{u}_{\mathcal{P}}^{(n+1)}$ is reconstructed from $p^{(n+1)}$ and, to account for the advection term in the concentration equation, the following advection equation is considered; it defines space-time test functions from chosen final values:

$$
\phi \partial_{t} v+\mathbf{u}_{\mathcal{P}}^{(n+1)} \cdot \nabla v=0 \quad \text { on }\left(t^{(n)}, t^{(n+1)}\right) \text {, with } v\left(\cdot, t^{(n+1)}\right) \text { given } .
$$

iii) Setting $\mathbf{U}_{\mathcal{P}}^{(n+1)}=A\left(\boldsymbol{x}, \Pi_{\mathcal{C}} c^{(n)}\right) \nabla_{\mathcal{P}} p^{(n+1)}$ and using a weighted trapezoid rule with weight $w \in[0,1]$ for the time-integration of the source term, $c^{(n+1)}$ satisfies

$$
\left\{\begin{array}{l}
\text { For all } z \in X_{\mathcal{C}}, \text { setting } v \text { the solution to }(7) \text { with } v\left(\cdot, t^{(n+1)}\right)=\Pi_{\mathcal{C}} z, \\
\int_{\Omega} \phi \Pi_{\mathcal{C}} c^{(n+1)} \Pi_{\mathcal{C}} z-\int_{\Omega} \phi \Pi_{\mathcal{C}} c^{(n)} v\left(t^{(n)}\right) \\
\quad+\delta t^{\left(n+\frac{1}{2}\right)} \int_{\Omega} \mathbf{D}\left(\boldsymbol{x}, \mathbf{U}_{\mathcal{P}}^{(n+1)}\right) \nabla_{\mathcal{C}} c^{(n+1)} \cdot \nabla_{\mathcal{C}} z \\
\quad+w \delta t^{\left(n+\frac{1}{2}\right)} \int_{\Omega} \Pi_{\mathcal{C}} c^{(n)} v\left(t^{(n)}\right) q_{n}^{-}+(1-w) \delta t^{\left(n+\frac{1}{2}\right)} \int_{\Omega} \Pi_{\mathcal{C}} c^{(n+1)} \Pi_{\mathcal{C}} z q_{n+1}^{-} \\
\quad=w \delta t^{\left(n+\frac{1}{2}\right)} \int_{\Omega} q_{n}^{+} v\left(t^{(n)}\right)+(1-w) \delta t^{\left(n+\frac{1}{2}\right)} \int_{\Omega} q_{n+1}^{+} \Pi_{\mathcal{C}} z,
\end{array}\right.
$$

where $q_{N}^{ \pm}=q_{N-1}^{ \pm}$if these quantities are defined by averages on time intervals (there is no time interval $\left(t^{(N)}, t^{(N+1)}\right)$ ).

Remark 3.2. Using a weighted trapezoid rule for the time discretisation of the reaction/source terms is essential to obtain an accurate numerical scheme $[2,11]$.

Defining the flow $F_{t}$ such that, for a.e. $\boldsymbol{x} \in \Omega$,

$$
\frac{d F_{t}(\boldsymbol{x})}{d t}=\frac{\mathbf{u}_{\mathcal{P}}^{(n+1)}\left(F_{t}(\boldsymbol{x})\right)}{\phi\left(F_{t}(\boldsymbol{x})\right)} \quad \text { for } t \in[-T, T], \quad F_{0}(\boldsymbol{x})=\boldsymbol{x},
$$

the solution to (7) is understood in the sense: for $\left.t \in\left(t^{(n)}, t^{(n+1)}\right)\right]$ and a.e. $\boldsymbol{x} \in$ $\Omega, v(\boldsymbol{x}, t)=v\left(F_{t^{(n+1)}-t}(\boldsymbol{x}), t^{(n+1)}\right)$. Hence, in $(8), v\left(\cdot, t^{(n)}\right)=\Pi_{\mathcal{C}} z\left(F_{\delta t^{(n+1 / 2)}}(\cdot)\right)$. Under Assumptions (2b) and (A4) below, the existence and uniqueness of the flow is discussed in Lemma 5.1. We note that $F_{t}$ depends on $n$ through $\mathbf{u}_{\mathcal{P}}^{(n+1)}$, but this dependency is not explicitly indicated when there is no risk of confusion.

The convergence theorem is established under the following assumptions. We show in Section 4 that various finite element and finite volume methods are given by GDs that satisfy these assumptions. 
(A1) $\left(\mathcal{P}_{m}\right)_{m \in \mathbb{N}}$ and $\left(\mathcal{C}_{m}^{T}\right)_{m \in \mathbb{N}}$ are coercive, GD-consistent and limit-conforming sequences of space-time GDs, and $\left(\mathcal{C}_{m}^{T}\right)_{m \in \mathbb{N}}$ is moreover compact. Denoting by $0=t_{m}^{(0)}<\cdots<t_{m}^{\left(N_{m}\right)}=T$ the time steps of $\mathcal{C}_{m}$, it is assumed that there exists $M_{t} \geq 0$ such that, for all $m \in \mathbb{N}$ and $n=1, \ldots, N-1$, $\delta t_{m}^{(n+1 / 2)} \leq M_{t} \delta t_{m}^{(n-1 / 2)}$.

(A2) There exists $M_{F} \geq 0$ such that, for all $m \in \mathbb{N}, z \in X_{\mathcal{C}_{m}}$, all $n=0, \ldots, N_{m}-$ 1 , and all $s \in[-T, T]$,

$$
\left\|\Pi_{\mathcal{C}_{m}} z\left(F_{s}\right)-\Pi_{\mathcal{C}_{m}} z\right\|_{L^{1}(\Omega)} \leq M_{F}|s|\left\|\mathbf{u}_{\mathcal{P}_{m}}^{(n+1)}\right\|_{L^{2}(\Omega)}\left\|\nabla_{\mathcal{C}_{m}} z\right\|_{L^{2}(\Omega)} .
$$

(A3) For all $m \in \mathbb{N}$ there is an interpolant $\mathcal{J}_{\mathcal{C}_{m}}: C^{\infty}(\bar{\Omega}) \rightarrow X_{\mathcal{C}_{m}}$ such that, for all $\varphi \in C^{\infty}(\bar{\Omega})$, as $m \rightarrow \infty, \nabla_{\mathcal{C}_{m}} \mathcal{J}_{\mathcal{C}_{m}} \varphi \rightarrow \nabla \varphi$ in $L^{4}(\Omega)^{d}$ and $\Pi_{\mathcal{C}_{m}} \mathcal{J}_{\mathcal{C}_{m}} \varphi \rightarrow \varphi$ in $L^{\infty}(\Omega)$.

(A4) There exists $M_{\text {div }}>0$ such that, for all $m \in \mathbb{N}$ and $n=0, \ldots, N_{m}-1$, $\mathbf{u}_{\mathcal{P}_{m}}^{(n+1)} \in H_{\text {div }}(\Omega)$ is piecewise polynomial on a mesh, $\mathbf{u}_{\mathcal{P}_{m}}^{(n+1)} \cdot \mathbf{n}=0$ on $\partial \Omega$, and $\left|\operatorname{div} \mathbf{u}_{\mathcal{P}_{m}}^{(n+1)}\right| \leq M_{\text {div }}$ on $\Omega$.

(A5) If $\left(p_{m}, c_{m}\right) \in X_{\mathcal{P}_{m}}^{N_{m}} \times X_{\mathcal{C}_{m}}^{N_{m}+1}$ is a solution to the GDM-ELLAM scheme with $\left(\mathcal{P}, \mathcal{C}^{T}\right)=\left(\mathcal{P}_{m}, \mathcal{C}_{m}^{T}\right)$ and $\mathbf{u}_{\mathcal{P}_{m}}: \Omega \times(0, T) \rightarrow \mathbb{R}^{d}$ is defined by $\mathbf{u}_{\mathcal{P}_{m}}(\cdot, t)=$ $\mathbf{u}_{\mathcal{P}_{m}}^{(n+1)}$ for all $t \in\left(t_{m}^{(n)}, t_{m}^{(n+1)}\right)$ and $n=0, \ldots, N_{m}-1$, then, when (A1)(A4) hold:

(a) $\left\|\mathbf{u}_{\mathcal{P}_{m}}\right\|_{L^{\infty}\left(0, T ; L^{2}(\Omega)\right)} \leq C_{m}\left\|\nabla_{\mathcal{P}_{m}} p_{m}\right\|_{L^{\infty}\left(0, T ; L^{2}(\Omega)\right)}$ with $\left(C_{m}\right)_{m \in \mathbb{N}}$ bounded.

(b) if $p \in L^{2}\left(0, T ; H^{1}(\Omega)\right)$ and $c \in L^{2}(\Omega \times(0, T))$ are such that, as $m \rightarrow \infty$, $\Pi_{\mathcal{D}_{m}} p_{m} \rightarrow \bar{p}, \Pi_{\mathcal{C}_{m}} c_{m} \rightarrow c$ and $\nabla_{\mathcal{P}_{m}} p_{m} \rightarrow \nabla p$ in $L^{2}(\Omega \times(0, T))$, then $\mathbf{u}_{\mathcal{P}_{m}} \rightarrow \mathbf{u}=-\frac{\mathbf{K}}{\mu(c)} \nabla p$ weakly in $L^{2}(\Omega \times(0, T))^{d}$.

Theorem 3.3 (Convergence of the GDM-ELLAM scheme). Under Assumptions (2) and (A1)-(A5), for any $m \in \mathbb{N}$ there is a unique $\left(p_{m}, c_{m}\right) \in X_{\mathcal{P}_{m}}^{N_{m}} \times X_{\mathcal{C}_{m}}^{N_{m}+1}$ solution of the GDM-ELLAM scheme with $\left(\mathcal{P}, \mathcal{C}^{T}\right)=\left(\mathcal{P}_{m}, \mathcal{C}_{m}^{T}\right)$. Moreover, up to a subsequence as $m \rightarrow \infty$,

- $\Pi_{\mathcal{P}_{m}} p_{m} \rightarrow p$ and $\nabla_{\mathcal{P}_{m}} p_{m} \rightarrow \nabla p$ weakly-* in $L^{\infty}\left(0, T ; L^{2}(\Omega)\right)$ and strongly in $L^{r}\left(0, T ; L^{2}(\Omega)\right)$ for all $r<\infty$,

- $\Pi_{\mathcal{C}_{m}} c_{m} \rightarrow c$ weakly-* in $L^{\infty}\left(0, T ; L^{2}(\Omega)\right)$ and strongly in $L^{r}\left(0, T ; L^{2}(\Omega)\right)$ for all $r<\infty$,

- $\nabla_{\mathcal{C}_{m}} c_{m} \rightarrow \nabla c$ weakly in $L^{2}(\Omega \times(0, T))^{d}$,

where $(p, c)$ is a weak solution of $(1)$.

Remark 3.4 (About the assumptions). Assumption (A1) is standard in analysis of gradient schemes, except for the assumption on the time steps, which is not very restrictive in practice (it is for example satisfied by uniform time steps, used in most numerical tests on (1), see e.g. [8,32]). Assumption (A2) is probably the most technical to check for specific methods; we however provide two results (Lemmas 5.3 and 5.5) which show that it is satisfied for a wide range of conforming or non-conforming methods. Assumption (A3) is satisfied by all standard interpolants associated with numerical methods for diffusion equations. Assumption (A4) is natural given the pressure equation (1a) and the boundedness assumption (2a) on $q^{+}$ and $q^{-}$. Finally, Assumption (A5) is also rather natural since it is expected that the 
reconstructed Darcy velocity $\mathbf{u}_{\mathcal{P}}$ is closely related to the reconstructed concentration $\Pi_{\mathcal{C}} c$ and pressure gradient $\nabla_{\mathcal{P}} p$.

Remark 3.5 (One GD per time step). In some particular cases, most notably the discretisation via mixed finite elements (see Section 4.1.1), the gradient discretisation $\mathcal{P}$ changes with each time step. Each equation (6) is written with a specific gradient discretisation $\mathcal{P}^{(n+1)}$. Hence, the choice $\mathcal{P}$ of a gradient discretisation for the pressure actually amounts to choosing a family $\mathcal{P}=\left(\mathcal{P}^{(i)}\right)_{i=1, \ldots, N}$. Theorem 3.3 remains valid provided that the coercivity, GD-consistency and limit-conformity of a sequence $\left(\mathcal{P}_{m}\right)_{m \in \mathbb{N}}=\left(\left(\mathcal{P}_{m}^{(i)}\right)_{i=1, \ldots, N_{m}}\right)_{m \in \mathbb{N}}$ of such families of $G D$ s are defined as in Definition 2.2 with

$$
C_{\mathcal{P}_{m}}=\max _{i=1, \ldots, N_{m}} C_{\mathcal{P}_{m}^{(i)}}, \quad S_{\mathcal{P}_{m}}=\max _{i=1, \ldots, N_{m}} S_{\mathcal{P}_{m}^{(i)}} \quad \text { and } \quad W_{\mathcal{P}_{m}}=\max _{i=1, \ldots, N_{m}} W_{\mathcal{P}_{m}^{(i)}}
$$

\section{Sample methods COVERED By The ANALYsis}

The ELLAM is a way to deal with the advection term in the concentration equation. Various numerical methods can be chosen to discretise the diffusion terms in this equation, as well as in the pressure equation. These methods correspond to selecting specific gradient discretisations $\mathcal{C}$ and $\mathcal{P}$. Here, we detail some of the GDs corresponding to methods used in the literature in conjunction with the ELLAM, and we show that they all satisfy the assumptions of Theorem 3.3. As a consequence, our convergence result applies to all these methods.

In the following, for simplicity of notations, we drop the index $m$ in the gradient discretisations and we consider Assumptions (A1)-(A5) 'as the mesh size and time step go to zero' (as opposed to 'as $m \rightarrow \infty$ ').

4.1. Conforming/mixed finite-element methods. When discretising the model (1) using finite element methods for the diffusion terms and the ELLAM for the advection term, it is natural to use a mixed method for the pressure equation and a conforming method for the concentration equation. The mixed method provides an appropriate Darcy velocity that can be used to build the ELLAM characteristics. This approach was considered in $[31,32]$. We show here that such a mixed/conforming FE-ELLAM scheme fits into our GDM-ELLAM framework, so that the convergence result of Theorem 3.3 applies to the schemes in the aforementioned references. Notice that, contrary to the convergence analysis done for example in [31], our convergence result relies on very weak regularity assumptions on the data and solution, that are usually satisfied in practical applications.

4.1.1. Description of the conforming and mixed FE GDs. Any conforming Galerkin approximation, which include conforming finite element methods (such as $\mathbb{P}_{k} \mathrm{FE}$ on simplices, or $\mathbb{Q}_{k} \mathrm{FE}$ on Cartesian grids), fits into the GDM framework. A finite-dimensional subspace $V_{h}$ of $H^{1}(\Omega)$ being chosen, we define $\left(X_{\mathcal{C}}, \Pi_{\mathcal{C}}, \nabla_{\mathcal{C}}\right)$ by $X_{\mathcal{C}}=V_{h}$ and, for $v \in V_{h}, \Pi_{\mathcal{C}} v=v$ and $\nabla_{\mathcal{C}} v=\nabla v$. The interpolant $\mathcal{I}_{\mathcal{C}}$ can be either chosen as the orthogonal projection on $V_{h}$, in the case of an abstract space, or as the standard nodal interpolant for specific FE spaces.

We now describe a gradient discretisation $\mathcal{P}$ corresponding to the $\mathbb{R T}_{0}$ mixed finite element method. The following construction can be extended to higher order 
$\mathbb{R T}_{k}$ finite elements [23]. A conforming simplicial or Cartesian mesh $\mathcal{M}$ being chosen, define

$$
\begin{aligned}
& \boldsymbol{V}_{h, 0}=\left\{\boldsymbol{v} \in H_{\mathrm{div}}(\Omega): \boldsymbol{v}_{\mid K} \in \mathbb{R}_{0}(K), \forall K \in \mathcal{M}, \boldsymbol{v} \cdot \mathbf{n}=0 \text { on } \partial \Omega\right\}, \\
& W_{h}=\left\{z \in L^{2}(\Omega): z_{\mid K} \text { constant }, \forall K \in \mathcal{M}\right\},
\end{aligned}
$$

where $\mathbb{R T}_{0}$ is the lowest order Raviart-Thomas space on the cell $K$ (the description of $\mathbb{R T}_{0}$ depends if this cell is a simplex or Cartesian cell). After choosing a diffusion tensor $\mathcal{A}$ - that is, a symmetric, uniformly bounded and coercive matrix-valued function $\Omega \rightarrow M_{d}(\mathbb{R})$ - a gradient discretisation $\mathcal{P}=\left(X_{\mathcal{P}}, \Pi_{\mathcal{P}}, \nabla_{\mathcal{P}}\right)$ is constructed by setting $X_{\mathcal{P}}=W_{h}$ and, for $z \in W_{h}, \Pi_{\mathcal{P}} z=z$. The reconstructed gradient $\nabla_{\mathcal{P}} z$ is defined as the solution to

$$
\begin{aligned}
& \mathcal{A} \nabla_{\mathcal{D}} z \in \boldsymbol{V}_{h, 0} \text { and, for all } \boldsymbol{w} \in \boldsymbol{V}_{h, 0}, \\
& \int_{\Omega} \boldsymbol{w}(\boldsymbol{x}) \cdot \nabla_{\mathcal{P}} z(\boldsymbol{x}) d \boldsymbol{x}=-\int_{\Omega} z(\boldsymbol{x}) \operatorname{div} \boldsymbol{w}(\boldsymbol{x}) d \boldsymbol{x} .
\end{aligned}
$$

The existence and uniqueness of $\nabla_{\mathcal{P}} z$ follows by applying the Riesz representation theorem in $\boldsymbol{V}_{h, 0}$ with the inner product $(\boldsymbol{w}, \boldsymbol{v}) \mapsto \int_{\Omega} \boldsymbol{w} \cdot \mathcal{A}^{-1} \boldsymbol{v} d \boldsymbol{x}$.

Taking $\mathcal{A}(\boldsymbol{x})=\frac{\mathbf{K}(\boldsymbol{x})}{\mu\left(\Pi_{\mathcal{C}} c^{(n)}(\boldsymbol{x})\right)}$, the scheme (6) is exactly an $\mathbb{R}_{0}$ mixed finite element discretisation of the pressure equation at the $n$-th time step. We notice here that $\mathcal{A}$, and thus the gradient discretisation $\mathcal{P}$ built above, changes with each time step; we are therefore in the context of Remark 3.5.

4.1.2. Assumptions (A1)-(A5). We show here that all required assumptions for Theorem 3.3 are satisfied by sequences of GDs as in Section 4.1.1.

Under usual mesh regularity properties, Assumption (A1) follows from $[16$, Chapters 8 and 9] (note that $W_{\mathcal{C}} \equiv 0$ and $C_{\mathcal{C}} \leq C_{P}$, where $C_{P}$ is the PoincaréWirtinger constant in $H^{1}(\Omega)$ ). For the GD $\mathcal{P}$ built on the $\mathbb{R T}_{0}$ mixed $\mathrm{FE}$, although the matrix $\mathcal{A}$ changes with each time step, it always remains uniformly bounded and coercive; the analysis in [23] thus shows that the notions of coercivity, GDconsistency and limit-conformity as in Remark 3.5 are verified.

Thanks to (2a), the standard Darcy velocity $\mathbf{u}_{\mathcal{P}}^{(n+1)}=-\frac{\mathbf{K}}{\mu\left(\Pi_{\mathcal{C}}{ }^{(n)}\right)} \nabla_{\mathcal{P}} p^{(n+1)}$ resulting from the $\mathbb{R T}_{0}$ discretisation of the pressure equation already satisfies Assumption (A4), and is therefore naturally used as tracking velocity. Assumption (A5)a) is trivially satisfied since $\left|\mathbf{u}_{\mathcal{P}}\right| \leq \Lambda_{A}\left|\nabla_{\mathcal{P}} p\right|$. Moreover, under (A1), if $\Pi_{\mathcal{C}} c \rightarrow c$ in $L^{2}(\Omega \times(0, T))$ as the mesh size and time step go to 0 , then $\widetilde{\Pi}_{\mathcal{C}} c$ also converges to $c$ in the same space (see, e.g., end of Section 7.1); thus, if $\nabla_{\mathcal{P}} p \rightarrow \nabla p$ in $L^{2}(\Omega \times(0, T))$, the assumption (2c) on $\mathbf{K} / \mu$ ensures that $\mathbf{u}_{\mathcal{P}}=-\frac{\mathbf{K}}{\mu\left(\widetilde{\Pi}_{\mathcal{C}} c\right)} \nabla_{\mathcal{P}} p$ strongly converges in $L^{2}(\Omega \times(0, T))$ to $\mathbf{u}=-\frac{\mathbf{K}}{\mu(c)} \nabla p$, which proves $\left.(\mathbf{A} \mathbf{5}) \mathrm{b}\right)$.

For $\mathcal{C}$ coming from a conforming finite element method, the standard nodal interpolation $\mathcal{J}_{\mathcal{C}}$ clearly satisfies (A3) (see [6, Theorem 4.4.20]). Finally, Assumption (A2) follows from Lemma 5.3 applied to $f=\Pi_{\mathcal{C}} z \in H^{1}(\Omega), \alpha=1$ and $r=2$.

4.2. Finite-volume based. A number of finite volume numerical schemes can be embedded in the gradient discretisation method [16]. Here, we focus on one particular method, the Hybrid Mimetic Mixed method (HMM) [17], which covers in particular the hybrid finite volume schemes [22], the mixed/hybrid Mimetic Finite Differences presented for example in [7], and the mixed finite volume method [15]. The HMM method was used in $[11,12]$ to discretise the diffusion terms in both the 
pressure and concentration equations, together with the ELLAM for the advection term. The analysis carried out here applies to many other numerical schemes based on piecewise-constant reconstructions, such as the VAG scheme, the MPFA-O FV method, mass-lumped FE methods or nodal Mimetic Finite Differences [16].

4.2.1. Description of the HMM gradient discretisation. Let us first introduce a few mesh-related notations. We consider a polytopal mesh $\mathfrak{T}=(\mathcal{M}, \mathcal{E}, \mathcal{P})$ of $\Omega \subset \mathbb{R}^{d}$ as in [16, Definition 7.2]. $\mathcal{M}$ is the set of polytopal cells (polygons in 2D, polyhedra in $3 \mathrm{D}), \mathcal{E}$ the set of faces (edges in $2 \mathrm{D}$ ) and $\mathcal{P}$ a set of one point $\boldsymbol{x}_{K}$ inside each cell $K \in \mathcal{M}$. No conformity is assumed on the mesh, which can therefore have hanging nodes, be locally refined, have non-convex cells, etc. For $K \in \mathcal{M}, \mathcal{E}_{K}$ denotes the set of faces of $K$ and, if $\sigma \in \mathcal{E}_{K}, D_{K, \sigma}$ is the convex hull of $\sigma$ and $\boldsymbol{x}_{K}, d_{K, \sigma}$ is the orthogonal distance between $\boldsymbol{x}_{K}$ and $\sigma, \mathbf{n}_{K, \sigma}$ is the outer unit normal to $\sigma$ and $\overline{\boldsymbol{x}}_{\sigma}$ is the center of mass of $\sigma$ (see Figure 1). It is assumed that each $K \in \mathcal{M}$ is star-shaped with respect to $\boldsymbol{x}_{K}$.

Figure 1. Notations inside a cell.

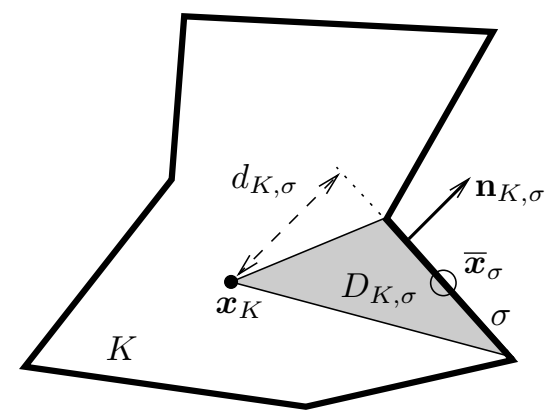

A spatial gradient discretisation $\mathcal{D}=\left(X_{\mathcal{D}}, \Pi_{\mathcal{D}}, \nabla_{\mathcal{D}}\right)$ and interpolant $\mathcal{I}_{\mathcal{D}}$ are then constructed by setting

$$
X_{\mathcal{D}}=\left\{v=\left(\left(v_{K}\right)_{K \in \mathcal{M}},\left(v_{\sigma}\right)_{\sigma \in \mathcal{E}}\right): v_{K} \in \mathbb{R}, v_{\sigma} \in \mathbb{R}\right\}
$$

(that is, there is one unknown per cell and one unknown per face),

$$
\begin{gathered}
\forall v \in X_{\mathcal{D}}, \forall K \in \mathcal{M}: \Pi_{\mathcal{D}} v=v_{K} \text { on } K \\
\forall \varphi \in L^{2}(\Omega): \mathcal{I}_{\mathcal{D}} \varphi=\left(\left(\varphi_{K}\right)_{K \in \mathcal{M}},\left(\varphi_{\sigma}\right)_{\sigma \in \mathcal{E}_{K}}\right) \in X_{\mathcal{D}} \\
\text { where } \varphi_{K}=\frac{1}{|K|} \int_{K} \varphi(\boldsymbol{x}) d \boldsymbol{x} \text { and } \varphi_{\sigma}=0
\end{gathered}
$$

(note that only $\Pi_{\mathcal{D}} \mathcal{I}_{\mathcal{D}}$ is of interest - see Definition 2.2 - so the value of the edge unknowns is irrelevant for $\mathcal{I}_{\mathcal{D}}$ ) and

$$
\begin{aligned}
& \forall v \in X_{\mathcal{D}}, \forall K \in \mathcal{M}, \forall \sigma \in \mathcal{E}_{K}, \\
& \nabla_{\mathcal{D}} v=\bar{\nabla}_{K} v+\frac{\sqrt{d}}{d_{K, \sigma}}\left[v_{\sigma}-v_{K}-\bar{\nabla}_{K} v \cdot\left(\overline{\boldsymbol{x}}_{\sigma}-\boldsymbol{x}_{K}\right)\right] \mathbf{n}_{K, \sigma} \text { on } D_{K, \sigma}, \\
& \text { where } \bar{\nabla}_{K} v=\frac{1}{|K|} \sum_{\sigma \in \mathcal{E}_{K}}|\sigma| v_{\sigma} \mathbf{n}_{K, \sigma} .
\end{aligned}
$$


Remark 4.1. In (11), $\bar{\nabla}_{K} v$ represents a consistent discretisation of the gradient, in the sense that if $\left(v_{\sigma}\right)_{\sigma \in \mathcal{E}_{K}}$ interpolate an affine mapping $A$ at the points $\left(\overline{\boldsymbol{x}}_{\sigma}\right)_{\sigma \in \mathcal{E}_{K}}$, then $\bar{\nabla}_{K} v=\nabla A$. The second part of $\nabla_{\mathcal{D}} v$, akin to the remainder of a discrete first order Taylor expansion, is a stabilisation term. More general forms of stabilisation can be chosen [18], but we do not describe them to simplify the presentation.

An HMM scheme for (1a) is obtained by writting (6) with $\mathcal{P}=\mathcal{D}$ constructed above. Such a scheme can be formulated as a finite volume scheme. Define, for $p \in X_{\mathcal{D}}$ and $K \in \mathcal{M}$, the fluxes $\left(\mathcal{F}_{K, \sigma}(p)\right)_{\sigma \in \mathcal{E}_{K}}$ by

$$
\forall v \in X_{\mathcal{D}}, \sum_{\sigma \in \mathcal{E}_{K}} \mathcal{F}_{K, \sigma}(p)\left(v_{K}-v_{\sigma}\right)=\int_{K} \mathcal{A}(\boldsymbol{x}) \nabla_{\mathcal{D}} p(\boldsymbol{x}) \cdot \nabla_{\mathcal{D}} v(\boldsymbol{x}) d \boldsymbol{x}
$$

with $\mathcal{A}$ a diffusion matrix. Then $p$ solves (6) with $\mathcal{P}=\mathcal{D}$ and $\mathcal{A}=A\left(\cdot, \Pi_{\mathcal{C}} c^{(n)}\right)$ if and only if $\int_{\Omega} \Pi_{\mathcal{D}} p=0$ and the corresponding fluxes satisfy the balance and conservativity relations, constitutive equations of finite volume schemes [14]:

$$
\begin{aligned}
& \forall K \in \mathcal{M}, \sum_{\sigma \in \mathcal{E}_{K}} \mathcal{F}_{K, \sigma}(p)=\int_{K}\left(q^{+}-q^{-}\right)(\boldsymbol{x}) d \boldsymbol{x}, \\
& \forall \sigma \text { face between two cells } K \text { and } L, \mathcal{F}_{K, \sigma}(p)+\mathcal{F}_{L, \sigma}(p)=0, \\
& \forall \sigma \text { face contained in } \partial \Omega, \mathcal{F}_{K, \sigma}(p)=0 .
\end{aligned}
$$

4.2.2. Assumptions (A1)-(A3). Let us define the mesh regularity parameter

$$
\varrho_{\mathfrak{T}}=\max _{K \in \mathcal{M}} \operatorname{Card}\left(\mathcal{E}_{K}\right)+\max _{K \in \mathcal{M}} \max _{\sigma \in \mathcal{E}_{K}} \frac{\operatorname{diam}\left(D_{K, \sigma}\right)}{\operatorname{inrad}\left(D_{K, \sigma}\right)},
$$

where $\operatorname{inrad}\left(D_{K, \sigma}\right)$ is the radius of the largest ball included in $D_{K, \sigma}$. Under a boundedness assumption on $\varrho_{\mathfrak{T}}$, the basic properties (A1) (with both $\mathcal{C}$ and $\mathcal{P}$ given by an HMM GD as in Section 4.2.1) follow from the results in [16, Chapter 12]. The appendix of [1] describes an interpolant $\mathcal{J}_{\mathcal{D}}$ and shows that it satisfies Assumption (A3).

Denoting by $Y_{\mathcal{M}}$ the space of piecewise constant functions on $\mathcal{M}$, we have $\Pi_{\mathcal{D}}\left(X_{\mathcal{D}}\right) \subset Y_{\mathcal{M}}$. Recalling the definition (32) of the discrete $H^{1}$-semi norm on $Y_{\mathcal{M}},\left[16\right.$, Lemma 12.9 and Remark 7.6] show that $\left|\Pi_{\mathcal{D}}\right|_{\mathcal{M}} \leq \beta_{\mathcal{D}}\left\|\nabla_{\mathcal{D}}\right\|_{L^{2}(\Omega)}$ with $\beta_{\mathcal{D}}$ depending only on an upper bound of $\varrho_{\mathfrak{T}}$ (this estimate is not specific to the HMM; it holds for all currently known GDs such that $\left.\Pi_{\mathcal{D}}\left(X_{\mathcal{D}}\right) \subset Y_{\mathcal{M}}\right)$. Assumption (A2) is therefore a consequence of Lemma 5.5, provided that the reconstructed Darcy velocity is piecewise polynomial (which is usually the case - see next section).

4.2.3. Reconstructed Darcy velocity and Assumptions (A4)-(A5). For methods like the HMM that produce piecewise-constant gradients $\nabla_{\mathcal{P}} p^{(n+1)}$ and/or piecewiseconstant concentration $\Pi_{\mathcal{C}} c^{(n)}$, the natural Darcy velocity $-\frac{\mathbf{K}}{\mu\left(\Pi_{\mathcal{C}} c^{(n)}\right)} \nabla_{\mathcal{P}} p^{(n+1)}$ does not belong to $H_{\text {div }}(\Omega)$. It is therefore not suitable to define the characteristics used in the ELLAM, and another velocity must be reconstructed to be used in (7). Finite-volume methods naturally produce numerical fluxes on the mesh faces, that satisfy the balance and conservativity relations (12)-(13). Such fluxes can be used to reconstruct a Darcy velocity in a Raviart-Thomas space on a sub-mesh of $\mathcal{M}$.

In $[11,12]$, this idea is applied to the HMM method on the sub-mesh of pyramids $\left(D_{K, \sigma}\right)_{K \in \mathcal{M}, \sigma \in \mathcal{E}_{K}}$. A velocity $\mathbf{u}_{\mathcal{P}}^{(n+1)} \in H_{\text {div }}(\Omega)$ is constructed from the pressure 
unknowns such that its restriction to each diamond $D_{K, \sigma}$ belongs to $\mathbb{R}_{0}$ and that, for each cell $K \in \mathcal{M}$,

$$
\begin{aligned}
& \text { For a.e. } \boldsymbol{x} \in K, \operatorname{div} \mathbf{u}_{\mathcal{P}}^{(n+1)}(\boldsymbol{x})=\frac{1}{|K|} \sum_{\sigma \in \mathcal{E}_{K}} \mathcal{F}_{K, \sigma}\left(p^{(n+1)}\right), \\
& \forall \sigma \in \mathcal{E}_{K}, \forall \boldsymbol{y} \in \sigma,|\sigma| \mathbf{u}_{\mathcal{P}}^{(n+1)}(\boldsymbol{y}) \cdot \mathbf{n}_{K, \sigma}=\mathcal{F}_{K, \sigma}\left(p^{(n+1)}\right) .
\end{aligned}
$$

Given the flux balance equation in (13), this reconstruction of $\mathbf{u}_{\mathcal{P}}^{(n+1)}$ satisfies Assumption (A4) with $M_{\mathrm{div}}=M_{q^{+}}+M_{q^{-}}($see $(2 \mathrm{a}))$.

Let us now establish the estimate on $\mathbf{u}_{\mathcal{P}}$ stated in (A5). In the following estimates, $A \lesssim B$ means that $A \leq C B$ with $C$ depending only on an upper bound of $\varrho \mathfrak{T}$, and of $\alpha_{A}$ and $\Lambda_{A}$ in (2c). Fix $K \in \mathcal{M}$. The relations (15) boil down to a linear system for internal fluxes in $K$ - that is, fluxes $\mathcal{F}_{\tau}$ on $\left(\partial D_{K, \sigma} \backslash \sigma\right)_{\sigma \in \mathcal{E}_{K}}$ - in which the right-hand side is $\left(\mathcal{F}_{K, \sigma}\left(p^{(n+1)}\right)\right)_{\sigma \in \mathcal{E}_{K}}$. Augmenting this system with a consistency relation or fixing the solution to be of minimal $\ell^{2}$ norm (see $[12,27])$ leads to a linear system $M_{K}\left(\mathcal{F}_{\tau}\right)_{\tau}=\left(\mathcal{F}_{K, \sigma}\left(p^{(n+1)}\right)\right)_{\sigma \in \mathcal{E}_{K}}$ with $M_{K}$ depending only on the number of faces of $K$, not on the geometry of this cell. Hence, $\sum_{\tau}\left|\mathcal{F}_{\tau}\right|^{2} \lesssim \sum_{\sigma \in \mathcal{E}_{K}}\left|\mathcal{F}_{K, \sigma}\left(p^{(n+1)}\right)\right|^{2}$. Due to the shape regularity assumption (which implies $|\tau|^{-1} \lesssim \operatorname{diam}(K) /|K|$ for any face $\tau$ of any pyramid $\left.D_{K, \sigma}\right)$ and by construction of $\mathbb{R T}_{0}$ functions, we infer that

$$
\begin{aligned}
\left\|\mathbf{u}_{\mathcal{P}}^{(n+1)}\right\|_{L^{2}\left(D_{K, \sigma}\right)}^{2} & \lesssim \sum_{\tau \subset \partial D_{K, \sigma}} \frac{\operatorname{diam}(K)}{|\tau|}\left|\mathcal{F}_{\tau}\right|^{2} \\
& \lesssim \frac{\operatorname{diam}(K)^{2}}{|K|} \sum_{\sigma \in \mathcal{E}_{K}}\left|\mathcal{F}_{K, \sigma}\left(p^{(n+1)}\right)\right|^{2} .
\end{aligned}
$$

Fix $\sigma \in \mathcal{E}_{K}$ and take, in (12), $v_{\sigma}=1$ and $v_{K}=v_{\sigma^{\prime}}=0$ if $\sigma \neq \sigma^{\prime}$. The definition (11) of $\nabla_{\mathcal{D}}$ easily shows that $\left|\nabla_{\mathcal{D}} v\right| \lesssim \operatorname{diam}(K)^{-1}$ and (12) therefore yields $\operatorname{diam}(K) \sum_{\sigma \in \mathcal{E}_{K}}\left|\mathcal{F}_{K, \sigma}\left(p^{(n+1)}\right)\right| \lesssim \int_{K}\left|\nabla_{\mathcal{D}} p^{(n+1)}(\boldsymbol{x})\right| d \boldsymbol{x}$. Hence, by the CauchySchwarz inequality,

$$
\frac{\operatorname{diam}(K)^{2}}{|K|} \sum_{\sigma \in \mathcal{E}_{K}}\left|\mathcal{F}_{K, \sigma}\left(p^{(n+1)}\right)\right|^{2} \lesssim\left\|\nabla_{\mathcal{D}} p^{(n+1)}\right\|_{L^{2}(K)}^{2}
$$

Combined with (16) this proves (A5)a).

Because of this bound, the weak convergence in (A5)b) follows if we can show that $\mathbf{u}_{\mathcal{P}}$ converges to $\mathbf{u}$ against any $\varphi \in C_{c}^{\infty}(\Omega \times(0, T))^{d}$. To establish this convergence, we first evaluate $\mathbf{u}_{\mathcal{P}}-\mathbf{U}_{\mathcal{P}}$, where $\mathbf{U}_{\mathcal{P}}=-\frac{\mathbf{K}}{\mu\left(\widetilde{\Pi}_{\mathcal{C}}\right)} \nabla_{\mathcal{D}} p$. Fix $\boldsymbol{\xi} \in \mathbb{R}^{d}$ and apply the divergence theorem between $\mathbf{u}_{\mathcal{P}}^{(n+1)} \in H_{\text {div }}(K)$ and the affine map $x \mapsto \boldsymbol{\xi} \cdot\left(\boldsymbol{x}-\boldsymbol{x}_{K}\right)$ to write

$$
\begin{aligned}
\int_{K} \mathbf{u}_{\mathcal{P}}^{(n+1)}(\boldsymbol{x}) \cdot \boldsymbol{\xi} d \boldsymbol{x}= & \int_{K} \mathbf{u}_{\mathcal{P}}^{(n+1)}(\boldsymbol{x}) \cdot \nabla\left(\boldsymbol{\xi} \cdot\left(\boldsymbol{x}-\boldsymbol{x}_{K}\right)\right) d \boldsymbol{x} \\
= & \sum_{\sigma \in \mathcal{E}_{K}} \int_{\sigma} \mathbf{u}_{\mathcal{P}}^{(n+1)}(\boldsymbol{y}) \cdot \mathbf{n}_{K, \sigma}\left[\boldsymbol{\xi} \cdot\left(\boldsymbol{y}-\boldsymbol{x}_{K}\right)\right] d \mathrm{~s}(\boldsymbol{y}) \\
& -\int_{K} \operatorname{div} \mathbf{u}_{\mathcal{P}}^{(n+1)}(\boldsymbol{x})\left[\boldsymbol{\xi} \cdot\left(\boldsymbol{x}-\boldsymbol{x}_{K}\right)\right] d \boldsymbol{x}
\end{aligned}
$$


Using then (15) and $\frac{1}{|\sigma|} \int_{\sigma} \boldsymbol{y} d \mathrm{~s}(\boldsymbol{y})=\overline{\boldsymbol{x}}_{\sigma}$ leads to

$$
\begin{aligned}
\int_{K} \mathbf{u}_{\mathcal{P}}^{(n+1)}(\boldsymbol{x}) \cdot \boldsymbol{\xi} d \boldsymbol{x}= & \sum_{\sigma \in \mathcal{E}_{K}} \mathcal{F}_{K, \sigma}\left(p^{(n+1)}\right) \boldsymbol{\xi} \cdot\left(\overline{\boldsymbol{x}}_{\sigma}-\boldsymbol{x}_{K}\right) \\
& -\int_{K} \operatorname{div} \mathbf{u}_{\mathcal{P}}^{(n+1)}(\boldsymbol{x})\left[\boldsymbol{\xi} \cdot\left(\boldsymbol{x}-\boldsymbol{x}_{K}\right)\right] d \boldsymbol{x} .
\end{aligned}
$$

Apply (12) with $v \in X_{\mathcal{D}}$ the interpolant of the linear mapping $\boldsymbol{x} \mapsto \boldsymbol{\xi} \cdot \boldsymbol{x}$, that is, $v_{K}=\boldsymbol{\xi} \cdot \boldsymbol{x}_{K}$ and $v_{\sigma}=\boldsymbol{\xi} \cdot \overline{\boldsymbol{x}}_{\sigma}$. The $\mathbb{P}_{1}$-exactness property of $\nabla_{\mathcal{D}}[16$, Lemma 12.8$]$ shows that $\nabla_{\mathcal{D}} v=\boldsymbol{\xi}$ and (12) thus gives

$$
\sum_{\sigma \in \mathcal{E}_{K}} \mathcal{F}_{K, \sigma}\left(p^{(n+1)}\right) \boldsymbol{\xi} \cdot\left(\overline{\boldsymbol{x}}_{\sigma}-\boldsymbol{x}_{K}\right)=\int_{K} \mathbf{U}_{\mathcal{P}}^{(n+1)}(\boldsymbol{x}) \cdot \boldsymbol{\xi} d \boldsymbol{x} .
$$

Combining with (17) and using the generality of $\boldsymbol{\xi}$ then yields

$$
\int_{K} \mathbf{u}_{\mathcal{P}}^{(n+1)}(\boldsymbol{x}) d \boldsymbol{x}-\int_{K} \mathbf{U}_{\mathcal{P}}^{(n+1)}(\boldsymbol{x}) d \boldsymbol{x}=-\int_{K} \operatorname{div} \mathbf{u}_{\mathcal{P}}^{(n+1)}(\boldsymbol{x})\left(\boldsymbol{x}-\boldsymbol{x}_{K}\right) d \boldsymbol{x} .
$$

Denoting by $\operatorname{Pr}_{\mathcal{M}}: L^{2}(\Omega)^{d} \rightarrow L^{2}(\Omega)^{d}$ the orthogonal projection on the piecewise constant functions on $\mathcal{M}$ (that is, $\left(P_{\mathcal{M}} f\right)_{\mid K}=\frac{1}{|K|} \int_{K} f(\boldsymbol{x}) d \boldsymbol{x}$ for all $\left.K \in \mathcal{M}\right)$, the above relation gives

$$
\left\|\operatorname{Pr}_{\mathcal{M}}\left(\mathbf{u}_{\mathcal{P}}^{(n+1)}-\mathbf{U}_{\mathcal{P}}^{(n+1)}\right)\right\|_{L^{1}(\Omega)} \leq h_{\mathcal{M}}\left\|\operatorname{div} \mathbf{u}_{\mathcal{P}}^{(n+1)}\right\|_{L^{1}(\Omega)}
$$

where $h_{\mathcal{M}}=\max _{K \in \mathcal{M}} \operatorname{diam}(K)$ is the mesh size. Owing to the boundedness of $\operatorname{div} \mathbf{u}_{\mathcal{P}}^{(n+1)}$, this shows that $\operatorname{Pr}_{\mathcal{M}}\left(\mathbf{u}_{\mathcal{P}}-\mathbf{U}_{\mathcal{P}}\right) \rightarrow 0$ in $L^{\infty}\left(0, T ; L^{1}(\Omega)\right)$ as $h_{\mathcal{M}} \rightarrow 0$. Take now $\varphi \in C_{c}^{\infty}(\Omega \times(0, T))^{d}$. Using the orthogonality property of $\operatorname{Pr}_{\mathcal{M}}$,

$$
\begin{aligned}
\left|\int_{\Omega \times(0, T)}\left(\mathbf{u}_{\mathcal{P}}-\mathbf{U}_{\mathcal{P}}\right) \cdot \boldsymbol{\varphi}\right| \\
\leq\left|\int_{\Omega \times(0, T)}\left(\mathbf{u}_{\mathcal{P}}-\mathbf{U}_{\mathcal{P}}\right) \cdot\left(\boldsymbol{\varphi}-\operatorname{Pr}_{\mathcal{M}} \boldsymbol{\varphi}\right)\right|+\left|\int_{\Omega \times(0, T)}\left(\mathbf{u}_{\mathcal{P}}-\mathbf{U}_{\mathcal{P}}\right) \cdot \operatorname{Pr}_{\mathcal{M}} \boldsymbol{\varphi}\right| \\
\leq\left\|\mathbf{u}_{\mathcal{P}}-\mathbf{U}_{\mathcal{P}}\right\|_{1} h_{\mathcal{M}}\|D \varphi\|_{\infty}+\left|\int_{\Omega \times(0, T)} \operatorname{Pr}_{\mathcal{M}}\left(\mathbf{u}_{\mathcal{P}}-\mathbf{U}_{\mathcal{P}}\right) \cdot \varphi\right| \\
\leq\left\|\mathbf{u}_{\mathcal{P}}-\mathbf{U}_{\mathcal{P}}\right\|_{1} h_{\mathcal{M}}\|D \varphi\|_{\infty}+\left\|\operatorname{Pr}_{\mathcal{M}}\left(\mathbf{u}_{\mathcal{P}}-\mathbf{U}_{\mathcal{P}}\right)\right\|\left\|_{1}\right\| \varphi \|_{\infty} .
\end{aligned}
$$

where $\|\cdot\|_{r}=\|\cdot\|_{L^{r}(\Omega \times(0, T))}$ and we used $\left\|\varphi-\operatorname{Pr}_{\mathcal{M}} \varphi\right\|_{\infty} \leq h_{\mathcal{M}}\|D \varphi\|_{\infty}$. The strong convergence of $\Pi_{\mathcal{C}} c$ ensures the strong convergence of $\widetilde{\Pi}_{\mathcal{C}} c$ (see end of Section 7.1); hence, the strong convergences assumed in (A5) show that $\mathbf{U}_{\mathcal{P}} \rightarrow \mathbf{u}=-\frac{\mathbf{K}}{\mu(c)} \nabla p$ in $L^{2}(\Omega \times(0, T))^{d}$. Since the right-hand side of (18) tends to 0 as $h_{\mathcal{M}} \rightarrow 0$, this concludes the proof that $\mathbf{u}_{\mathcal{P}} \rightarrow \mathbf{u}$ weakly in $L^{2}(\Omega \times(0, T))^{d}$ as the mesh size and time step tend to 0 .

\section{Properties of the Flow}

A few properties on the solution of the characteristic equation (9) are established here. To simplify the notations, we set $\mathbf{u}_{\mathcal{P}}^{(n+1)}=\mathbf{V}$. Hence, for $\boldsymbol{x} \in \Omega, t \mapsto F_{t}(\boldsymbol{x})$ solves

$$
\frac{d F_{t}(\boldsymbol{x})}{d t}=\frac{\mathbf{V}\left(F_{t}(\boldsymbol{x})\right)}{\phi\left(F_{t}(\boldsymbol{x})\right)} \quad \text { for } t \in[-T, T], \quad F_{0}(\boldsymbol{x})=\boldsymbol{x} .
$$


Associated with the flow equation (19) is the advection equation

$$
\phi \partial_{t} w+\mathbf{V} \cdot \nabla w=0 .
$$

A function $w$ is a solution to such an equation if it satisfies, for all $s, t \in[-T, T]$ such that $s-t \in[-T, T]$ and for a.e. $\boldsymbol{x} \in \Omega, w(\boldsymbol{x}, t)=w\left(F_{s-t}(\boldsymbol{x}), s\right)$.

These flow and advection equations will be studied under the assumptions (2b) on $\phi$ and (A4) on V. Upon considering a common sub-mesh of the meshes considered in these assumptions there is no loss in generality in assuming that the meshes for $\phi$ and $\mathbf{V}$ are the same. In other words, our leading assumption here is: there is a mesh $\mathcal{M}$ (that is, a partition of $\Omega$ into polygonal/polyhedral cells) such that

$$
\begin{aligned}
& \phi \text { is piecewise smooth on } \mathcal{M} \text { and } \phi_{*} \leq \phi \leq \phi^{*}, \\
& \mathbf{V} \in H_{\text {div }}(\Omega) \text { is piecewise polynomial on } \mathcal{M}, \\
& |\operatorname{div} \mathbf{V}| \leq \Gamma_{\text {div }} \text { and } \mathbf{V} \cdot \mathbf{n}=0 \text { on } \partial \Omega
\end{aligned}
$$

Lemma 5.1 (The flow is well-defined). Under Assumption (21), there exists a closed set $\mathcal{C} \subset \Omega$ with zero Lebesgue measure such that, for any $\boldsymbol{x} \in \Omega \backslash \mathcal{C}$, there is a unique Lipschitz-continuous map $t \in[-T, T] \mapsto F_{t}(\boldsymbol{x}) \in \Omega \backslash \mathcal{C}$ that satisfies (19) (except at an at most countable number of times for the ODE). Moreover, $F_{t}$ has classical flows properties: for all $t \in[-T, T], F_{t}: \Omega \backslash \mathcal{C} \rightarrow \Omega \backslash \mathcal{C}$ is a locally Lipschitzcontinuous homeomorphism (which can thus be used for changes of variables in integrals), and $F_{t+s}=F_{t} \circ F_{s}$ for all $s, t \in[-T, T]$ such that $s+t \in[-T, T]$.

Proof. By smoothness of $\mathbf{V}$ and $\phi$ in each cell, the flow $t \mapsto F_{t}(\boldsymbol{x})$ of $\mathbf{V} / \phi$ can clearly be defined until it reaches a cell boundary. Assume that it reaches at a time $t=t_{\sigma}$ a cell boundary at a point $\boldsymbol{y}$ that is not a vertex or on an edge of the cell (we use here the 3D nomenclature), that is, $\boldsymbol{y}$ is in the relative interior of a face $\sigma$. Denote by $H_{1}$ and $H_{2}$ the two half-spaces on each side of $\sigma$, and by $\mathbf{n}_{\sigma}$ the normal to $\sigma$ from $H_{1}$ to $H_{2}$. Since $\mathbf{V} \in H_{\text {div }}(\Omega), \mathbf{V} \cdot \mathbf{n}_{\sigma}$ is continuous across $\sigma$. The function $\phi$ being positive, it means that the sign, if not the value, of $(\mathbf{V} / \phi) \cdot \mathbf{n}_{\sigma}$ is continuous across $\sigma$. Assuming for example that $(\mathbf{V} / \phi)_{\mid H_{1}}(\boldsymbol{y}) \cdot \mathbf{n}_{\sigma}>0$, then the flow arrives at $\boldsymbol{y}$ from $H_{1}$ and, $(\mathbf{V} / \phi)_{\mid H_{2}}(\boldsymbol{y}) \cdot \mathbf{n}_{\sigma}$ being also strictly positive, $t \mapsto F_{t}(\boldsymbol{x})$ can be restarted from $\left(t_{\sigma}, \boldsymbol{y}\right)$ by considering $(\mathbf{V} / \phi)_{\mid H_{2}}$ (which drives the flow into $H_{2}$ ). Note that the $H_{\text {div }}$-property of $\mathbf{V}$ is essential here to ensure that the flow can indeed be continued into $H_{2}$, and that the values of $\mathbf{V} / \phi$ at $\boldsymbol{y}$ from $H_{1}$ and $H_{2}$ do not simultaneously drive the flow in the other domain, thus freezing it at $\boldsymbol{y}$.

Following this process, the flow can be continued as long as it does not cross (or starts from) a vertex/edge or, for a face $\sigma$, the set $Z_{\sigma}=\left\{\boldsymbol{y} \in \sigma: \mathbf{V}(\boldsymbol{y}) \cdot \mathbf{n}_{\sigma}=0\right\}$. Let $\mathcal{C}$ be the set consisting of all $\boldsymbol{x} \in \Omega$ whose flow arrive (or starts from) at a vertex/edge, or one of the sets $Z_{\sigma}$. The set $\mathcal{C}$ can be obtained by tracing back on $[-T, T]$, following the process above, the vertices, edges or sets $Z_{\sigma}$ (until the flow can no longer be constructed, that is, the tracing-back process arrives on a vertex, edge or a set $Z_{\sigma^{\prime}}$ ). Since each such set is closed, $\mathcal{C}$ is closed. Moreover, vertices and edges have dimension $d-2$ or less, and are therefore traced-back by the flow into sets of zero $d$-dimensional measure. Consider now a set $Z_{\sigma}$. Since $\mathbf{V} \cdot \mathbf{n}_{\sigma}$ is a polynomial, either $Z_{\sigma}=\sigma$ or $Z_{\sigma}$ has dimension $d-2$ or less. In the latter case, as for vertices/edges, its traced-back set has zero $d$-dimensional measure. If $Z_{\sigma}=\sigma$, then $\mathbf{V}$ is parallel to $\sigma$ (whatever the side we consider for the values of $\mathbf{V}$ ) and the traced-back region of $Z_{\sigma}$ is contained in $\bar{\sigma}$, which has zero $d$-dimensional measure. 
Hence, $\mathcal{C}$ has zero $d$-dimensional measure. This reasoning also shows that the flow never crosses the boundary of $\Omega$, since $\mathbf{V} \cdot \mathbf{n}=0$ on $\partial \Omega$.

This construction ensures that, for all $\boldsymbol{x} \notin \mathcal{C}$, the flow $t \mapsto F_{t}(\boldsymbol{x}) \in \Omega \backslash \mathcal{C}$ is well-defined on $[-T, T]$, satisfies the ODEs except at a countable number of points (where it intersects faces), is Lipschitz-continuous (since it is globally continuous and Lipschitz inside each cell, with a Lipschitz constant bounded by $\left.\|\mathbf{V}\|_{L^{\infty}(\Omega)} / \phi_{*}\right)$, and satisfies the flow property $F_{t+s}=F_{t} \circ F_{s}$. To see that it is locally Lipschitz on $\Omega \backslash \mathcal{C}$ with respect to its base point $\boldsymbol{x}$, we simply have to notice that for $\boldsymbol{x} \notin \mathcal{C}$, by construction of $\mathcal{C}$, there is a ball $B(\boldsymbol{x}, \theta)$ centered at $\boldsymbol{x}$ such that, for any $\boldsymbol{y} \in$ $B(\boldsymbol{x}, \theta)$, the flow $t \mapsto F_{t}(\boldsymbol{y})$ travels into the same cells and crosses the same faces as $t \mapsto F_{t}(\boldsymbol{x})$. Since, in each cell, the flow is Lipschitz-continuous w.r.t. its base point with a uniform Lipschitz constant (because $\mathbf{V}$ and $\phi$ are smooth in each cell, with bounded derivatives), gluing the Lipschitz estimate thanks to the flow property we can check that $\boldsymbol{y} \mapsto F_{t}(\boldsymbol{y})$ is Lipschitz continuous on $B(\boldsymbol{x}, \theta)$. Note that because the open set $\Omega \backslash \mathcal{C}$ can be disconnected, this does not prove a global Lipschitz property of the flow.

The homoeomorphism property follows from the flow property which shows that, on $\Omega \backslash \mathcal{C}, F_{t} \circ F_{-t}=F_{0}=$ Id.

Let us now establish some relations and estimates on this flow.

Lemma 5.2 (Estimates on the flow). Under Assumptions (21), for a.e. $\boldsymbol{x} \in \Omega$ and all $s \in[-T, T]$, denoting by $J F_{t}$ the Jacobian determinant of $F_{t}$,

$$
\int_{0}^{s}\left|J F_{t}(\boldsymbol{x})\right|(\operatorname{div} \mathbf{V}) \circ F_{t}(\boldsymbol{x}) d t=\phi\left(F_{s}(\boldsymbol{x})\right)\left|J F_{s}(\boldsymbol{x})\right|-\phi(\boldsymbol{x})
$$

and

$$
\left|J F_{s}(\boldsymbol{x})\right| \leq C_{1}(s):=\frac{\phi^{*}}{\phi_{*}} \exp \left(\frac{\Gamma_{\text {div }}}{\phi_{*}}|s|\right) .
$$

Moreover, let $w \geq 0$ be a solution of (20). Then, for all $s, t \in[-T, T]$ such that $s-t \in[-T, T]$,

$$
\int_{\Omega} \phi(\boldsymbol{x}) w(\boldsymbol{x}, t-s) d \boldsymbol{x} \leq\left(1+\frac{\Gamma_{\mathrm{div}} C_{1}(T)}{\phi_{*}}|s|\right) \int_{\Omega} \phi(\boldsymbol{x}) w(\boldsymbol{x}, t) d \boldsymbol{x}
$$

and

$$
\int_{\Omega} w(\boldsymbol{x}, t-s) d \boldsymbol{x} \leq \frac{C_{1}(T)}{\phi_{*}} \int_{\Omega} \phi(\boldsymbol{x}) w(\boldsymbol{x}, t) d \boldsymbol{x}
$$

\section{Proof.}

Step 1: we establish the following generalised Liouville formula: for any measurable set $A \subset \Omega$,

$$
\frac{d}{d t} \int_{F_{t}(A)} \phi(\boldsymbol{y}) d \boldsymbol{y}=\int_{F_{t}(A)} \operatorname{div} \mathbf{V}(\boldsymbol{y}) d \boldsymbol{y}
$$

where the time derivative $\frac{d}{d t}$ is taken in the sense of distributions (this also shows that the function $t \mapsto \int_{F_{t}(A)} \phi(\boldsymbol{y}) d \boldsymbol{y}$ belongs to $\left.W^{1,1}(-T, T)\right)$.

Let $v_{0} \in C_{c}^{\infty}(\Omega)$ and set $v(\boldsymbol{x}, t)=v_{0}\left(F_{-t}(\boldsymbol{x})\right)$. Then $v$ is Lipschitz-continuous with respect to $t$ and, by the flow property, $v(\boldsymbol{x}, t)=v\left(F_{s-t}(\boldsymbol{x}), s\right)$. Hence,

$$
\partial_{t} v(\boldsymbol{x}, t)=\nabla v\left(F_{s-t}(\boldsymbol{x}), s\right) \cdot \frac{d}{d t}\left(F_{s-t}(\boldsymbol{x})\right)=-\nabla v\left(F_{s-t}(\boldsymbol{x}), s\right) \cdot \frac{\mathbf{V}\left(F_{s-t}(\boldsymbol{x})\right)}{\phi\left(F_{s-t}(\boldsymbol{x})\right)} .
$$


Given the piecewise regularity assumptions on $\mathbf{V}$ and $\phi$, for a.e. $\boldsymbol{x} \in \Omega$ we can let $s \rightarrow t$ in the above relation to find $\partial_{t} v(\boldsymbol{x}, t)=-\nabla v(\boldsymbol{x}, t) \cdot \frac{\mathbf{V}(\boldsymbol{x})}{\phi(\boldsymbol{x})}$. Hence, since $\mathbf{V} \in H_{\text {div }}(\Omega)$ with $\mathbf{V} \cdot \mathbf{n}=0$ on $\partial \Omega$,

$$
\begin{aligned}
\frac{d}{d t} \int_{\Omega} \phi(\boldsymbol{x}) v(\boldsymbol{x}, t) d \boldsymbol{x}=\int_{\Omega} \phi & (\boldsymbol{x}) \partial_{t} v(\boldsymbol{x}, t) d \boldsymbol{x} \\
& =-\int_{\Omega} \nabla v(\boldsymbol{x}, t) \cdot \mathbf{V}(\boldsymbol{x}) d \boldsymbol{x}=\int_{\Omega} v(\boldsymbol{x}, t) \operatorname{div} \mathbf{V}(\boldsymbol{x}) d \boldsymbol{x} .
\end{aligned}
$$

Let us now take a sequence $\left(v_{0}^{(n)}\right)_{n \in \mathbb{N}}$ in $C_{c}^{\infty}(\Omega)$ that converges a.e. on $\Omega$ to the characteristic function $\mathbf{1}_{A}$ of $A$, and such that $0 \leq v_{0}^{(n)} \leq 1$. The relation above yields

$$
\frac{d}{d t} \int_{\Omega} \phi(\boldsymbol{x}) v_{0}^{(n)}\left(F_{-t}(\boldsymbol{x})\right) d \boldsymbol{x}=\int_{\Omega} v_{0}^{(n)}\left(F_{-t}(\boldsymbol{x})\right) \operatorname{div} \mathbf{V}(\boldsymbol{x}) d \boldsymbol{x} .
$$

As $n \rightarrow \infty$, the right-hand side converges (by dominated convergence) to

$$
\int_{\Omega} \mathbf{1}_{A}\left(F_{-t}(\boldsymbol{x})\right) \operatorname{div} \mathbf{V}(\boldsymbol{x}) d \boldsymbol{x}=\int_{F_{t}(A)} \operatorname{div} \mathbf{V}(\boldsymbol{x}) d \boldsymbol{x} .
$$

The sequence of mappings $t \mapsto \int_{\Omega} \phi(\boldsymbol{x}) v_{0}^{(n)}\left(F_{-t}(\boldsymbol{x})\right) d \boldsymbol{x}$ converge pointwise to

$$
t \mapsto \int_{\Omega} \phi(\boldsymbol{x}) \mathbf{1}_{A}\left(F_{-t}(\boldsymbol{x})\right) d \boldsymbol{x}=\int_{F_{t}(A)} \phi(\boldsymbol{x}) d \boldsymbol{x},
$$

while remaining bounded. Hence, they converge weakly-* in $L^{\infty}(-T, T)$. We can therefore pass to the distributional limit in (27) to see that (26) holds.

Step 2: estimates on $J F_{t}$.

Set $A=B(\boldsymbol{x}, r)$ a ball of center $\boldsymbol{x}$ and radius $r$ contained in $\Omega$. Integrating (26) with respect to time from 0 to $s$ and using a change of variables $\boldsymbol{y}=F_{-t}(\boldsymbol{x})$, we obtain

$$
\begin{aligned}
\int_{B(\boldsymbol{x}, r)} \phi\left(F_{s}(\boldsymbol{y})\right)\left|J F_{s}(\boldsymbol{y})\right| d \boldsymbol{y}-\int_{B(\boldsymbol{x}, r)} & \phi(\boldsymbol{y}) d \boldsymbol{y} \\
& =\int_{0}^{s} \int_{B(\boldsymbol{x}, r)}\left|J F_{t}(\boldsymbol{y})\right|(\operatorname{div} \mathbf{V}) \circ F_{t}(\boldsymbol{y}) d t d \boldsymbol{y} .
\end{aligned}
$$

Dividing by the measure of $B(\boldsymbol{x}, r)$ and taking the limit as $r \rightarrow 0$, we obtain (22) for a.e. $\boldsymbol{x} \in \Omega$, due to the piecewise smoothness of $\mathbf{V}$ and $\phi$.

Assume to simplify the writing that $s \geq 0$ and use the assumption on $\operatorname{div} \mathbf{V}$ to deduce from $(22)$ that $\phi\left(F_{s}(\boldsymbol{x})\right)\left|J F_{s}(\boldsymbol{x})\right|-\phi(\boldsymbol{x}) \leq \Gamma_{\operatorname{div}} \int_{0}^{s}\left|J F_{t}(\boldsymbol{x})\right| d t$, and thus that

$$
\left|J F_{s}(\boldsymbol{x})\right| \leq \frac{\phi^{*}}{\phi_{*}}+\frac{\Gamma_{\mathrm{div}}}{\phi_{*}} \int_{0}^{s}\left|J F_{t}(\boldsymbol{x})\right| d t .
$$

Use then Gronwall's inequality to obtain (23).

Step 3: Estimates on $w$.

We recall that $w(\boldsymbol{x}, t-s)=w\left(F_{s}(\boldsymbol{x}), t\right)$. Hence, a change of variables and $(22)$ yield

$$
\begin{aligned}
\int_{\Omega} \phi(\boldsymbol{x}) w(\boldsymbol{x}, t-s) d \boldsymbol{x} & =\int_{\Omega} \phi(\boldsymbol{x}) w\left(F_{s}(\boldsymbol{x}), t\right) d \boldsymbol{x}=\int_{\Omega} w(\boldsymbol{y}, t) \phi\left(F_{-s}(\boldsymbol{y})\right)\left|J F_{-s}(\boldsymbol{y})\right| d \boldsymbol{y} \\
& =\int_{\Omega} w(\boldsymbol{y}, t)\left(\phi(\boldsymbol{y})+\int_{0}^{-s}\left|J F_{\rho}(\boldsymbol{y})\right|(\operatorname{div} \mathbf{V}) \circ F_{\rho}(\boldsymbol{y}) d \rho\right) d \boldsymbol{y}
\end{aligned}
$$


Estimate (24) follows by writing, thanks to (23), for a.e. $\boldsymbol{y} \in \Omega$,

$$
\left|\int_{0}^{-s}\right| J F_{\rho}(\boldsymbol{y})\left|(\operatorname{div} \mathbf{V}) \circ F_{\rho}(\boldsymbol{y}) d \rho\right| \leq \Gamma_{\operatorname{div}} C_{1}(T)|s| \leq \frac{\Gamma_{\operatorname{div}} C_{1}(T)}{\phi_{*}}|s| \phi(\boldsymbol{y}) .
$$

To establish (25), we simply write, still using a change of variables,

$$
\int_{\Omega} w(\boldsymbol{x}, t-s) d \boldsymbol{x}=\int_{\Omega} w\left(F_{s}(\boldsymbol{x}), t\right) d \boldsymbol{x}=\int_{\Omega} w(\boldsymbol{y}, t)\left|J F_{-s}(\boldsymbol{y})\right| d \boldsymbol{y}
$$

and we use (23) and $\phi \geq \phi_{*}$ to conclude.

The following lemma is used to prove that conforming discretisations satisfy Assumption (A2) (see Section 4.1.2), and to establish convergence properties, as the time step tends to 0, of functions transported by the flow (see Lemma 5.7).

Lemma 5.3 (Translation estimate for Sobolev functions). Under Assumption (21), let $F_{t}$ be the flow defined by (19), and let $r, \alpha \in[1, \infty]$ be such that $\frac{1}{\alpha}=\frac{1}{2}+\frac{1}{r}$. Then, for any $f \in W^{1, r}(\Omega)$ and $s \in[-T, T]$,

$$
\left\|f\left(F_{s}\right)-f\right\|_{L^{\alpha}(\Omega)} \leq \frac{C_{1}(T)^{1 / \alpha}}{\phi_{*}}|s|\|\mathbf{V}\|_{L^{2}(\Omega)}\|\nabla f\|_{L^{r}(\Omega)},
$$

where $C_{1}(T)=\frac{\phi^{*}}{\phi_{*}} \exp \left(\frac{\Gamma_{\mathrm{div}} T}{\phi_{*}}\right)$ as in $(23)$.

Proof. By density it suffices to prove the estimate for $f \in C^{1}(\bar{\Omega})$ (in the case $r=\infty$, we first establish it for $r<\infty$ and corresponding $\alpha_{r}$, using the density of smooth functions in $W^{1, r}$, and then let $\left.r \rightarrow \infty\right)$. For a.e. $\boldsymbol{x} \in \Omega$,

$$
\begin{aligned}
f\left(F_{s}(\boldsymbol{x})\right)-f(\boldsymbol{x})=\int_{0}^{s} \frac{d}{d t} f\left(F_{t}(\boldsymbol{x})\right) d t & =\int_{0}^{s} \nabla f\left(F_{t}(\boldsymbol{x})\right) \cdot \frac{d F_{t}(\boldsymbol{x})}{d t} d t \\
& =\int_{0}^{s} \nabla f\left(F_{t}(\boldsymbol{x})\right) \cdot \frac{\mathbf{V}\left(F_{t}(\boldsymbol{x})\right)}{\phi\left(F_{t}(\boldsymbol{x})\right)} d t
\end{aligned}
$$

Take the absolute value, the power $\alpha$ (using Jensen's inequality) and integrate over $\Omega$. Using $\phi \geq \phi_{*}$ and applying a change of variables $\boldsymbol{y}=F_{t}(\boldsymbol{x})$ along with (23), this leads to

$$
\begin{aligned}
\int_{\Omega}\left|f\left(F_{s}(\boldsymbol{x})\right)-f(\boldsymbol{x})\right|^{\alpha} d \boldsymbol{x} & \leq \frac{|s|^{\alpha-1}}{\phi_{*}^{\alpha}} \int_{\Omega} \int_{[0, s]}\left|\nabla f\left(F_{t}(\boldsymbol{x})\right)\right|^{\alpha}\left|\mathbf{V}\left(F_{t}(\boldsymbol{x})\right)\right|^{\alpha} d t d \boldsymbol{x} \\
& \leq \frac{|s|^{\alpha-1}}{\phi_{*}^{\alpha}} \int_{[0, s]}\left(\int_{\Omega}\left|\nabla f\left(F_{t}(\boldsymbol{x})\right)\right|^{\alpha}\left|\mathbf{V}\left(F_{t}(\boldsymbol{x})\right)\right|^{\alpha} d \boldsymbol{x}\right) d t \\
& \leq \frac{C_{1}(T)|s|^{\alpha}}{\phi_{*}^{\alpha}} \int_{\Omega}|\nabla f(\boldsymbol{y})|^{\alpha}|\mathbf{V}(\boldsymbol{y})|^{\alpha} d \boldsymbol{y} .
\end{aligned}
$$

The proof is complete by applying Hölder's estimate with exponents $r / \alpha$ and $2 / \alpha$, and by taking the power $1 / \alpha$ of the resulting inequality.

We now want to establish a similar result but for piecewise-constant functions. This will be useful to establish that discretisations based on piecewise-constant approximations, such as most FV methods, satisfy Assumption (A2). Before stating this lemma, we need a preliminary result.

Lemma 5.4 (Volume covered by a face transported by the flow). Under Assumption (21), let $F_{t}$ be the flow defined by (19). Let $\sigma$ be a face of the mesh over which $\mathbf{V}$ and $\phi$ are piecewise smooth. Let $V_{t}=\left|F_{[0, t]}(\sigma)\right|$ be the volume of the region 
covered by $\sigma$ when transported over $[0, t]$ by the flow, that is, $V_{t}=\mid\left\{F_{s}(\boldsymbol{y}): s \in\right.$ $[0, t], \boldsymbol{y} \in \sigma\} \mid$. Then

$$
\forall t \in[-T, T], V_{t} \leq \frac{C_{1}(T)}{\phi_{*}}|t| \int_{\sigma}\left|\mathbf{V}(\boldsymbol{y}) \cdot \mathbf{n}_{\sigma}\right| d \mathrm{~s}(\boldsymbol{y}),
$$

where $C_{1}(T)$ is given by (23) and $\mathbf{n}_{\sigma}$ is a normal to $\sigma$.

Proof. Notice first that since $\mathbf{V} \in H_{\text {div }}(\Omega)$, the normal components of $\mathbf{V}$ across the faces of the mesh are continuous, and thus $\left|\mathbf{V}(\boldsymbol{y}) \cdot \mathbf{n}_{\sigma}\right|$ is independent of the side of $\sigma$ chosen to compute $\mathbf{V}$. Without loss of generality, we assume $t \geq 0$.

If the face $\sigma$ is such that $Z_{\sigma}:=\left\{\boldsymbol{y} \in \sigma: \mathbf{V}(\boldsymbol{y}) \cdot \mathbf{n}_{\sigma}=0\right\}=\sigma$, then even though $\sigma \subset \mathcal{C}$ (see Lemma 5.1 and its proof), we clearly have $V_{t}=0$ since each point on the face is transported inside the face to one of its vertex/edge, which are $(d-2)$-dimensional objects then transported by the flow onto null sets in $\Omega$ (whatever side of $\sigma$ chosen to compute $\mathbf{V}$ and $\phi$ ). Hence, (28) holds for such faces.

Let us now assume that $Z_{\sigma} \neq \sigma$. Then, since $\mathbf{V} \cdot \mathbf{n}_{\sigma}$ is polynomial, $Z_{\sigma}$ is a negligible set in $\sigma$ for the $(d-1)$-dimensional measure and $F_{t}(\boldsymbol{y})$ is defined for all $\boldsymbol{y} \in \sigma \backslash Z_{\sigma}$. Since $F_{[0, t+h]}(\sigma)=F_{[0, t]}(\sigma) \cup F_{(t, t+h]}(\sigma)$, the flow property, a change of variables and (23) yield

$$
\begin{aligned}
V_{t+h}-V_{t}=\left|F_{(t, t+h]}(\sigma)\right| & =\left|F_{t}\left(F_{(0, h]}(\sigma)\right)\right| \\
& =\int_{F_{(0, h]}(\sigma)}\left|J F_{t}(\boldsymbol{y})\right| d \boldsymbol{y} \leq C_{1}(T)\left|F_{(0, h]}(\sigma)\right| .
\end{aligned}
$$

Choose an orthonormal basis of $\mathbb{R}^{d}$ such that $\sigma \subset\{0\} \times \mathbb{R}^{d-1}$ and $\mathbf{n}_{\sigma}=(1,0, \ldots, 0)$, and define $G: \mathbb{R} \times \sigma \rightarrow \mathbb{R}^{d}$ by $G(t, \boldsymbol{y})=F_{t}(\boldsymbol{y})$. Using the area formula [20, Theorem 1] we have

$$
\begin{array}{r}
\left|F_{(0, h]}(\sigma)\right|=\int_{\mathbb{R}^{d}} \mathbf{1}_{G((0, h] \times \sigma))}(\boldsymbol{x}) d \boldsymbol{x} \leq \int_{\mathbb{R}^{d}} \operatorname{Card}\left[((0, h] \times \sigma) \cap G^{-1}(\{\boldsymbol{x}\})\right](\boldsymbol{x}) d \boldsymbol{x} \\
=\int_{(0, h] \times \sigma}|J G(t, \boldsymbol{y})| d t d \mathrm{~s}(\boldsymbol{y})=\int_{0}^{h}\left(\int_{\sigma}|J G(t, \boldsymbol{y})| d \mathrm{~s}(\boldsymbol{y})\right) d t
\end{array}
$$

where $J G$ is the Jacobian determinant of $G$. Given the choice of basis in the range of $G$,

$$
\begin{aligned}
J G(t, \boldsymbol{y}) & =\operatorname{det}\left[\begin{array}{llll}
\frac{\partial G}{\partial t}(t, \boldsymbol{y}) & \frac{\partial G}{\partial y_{1}}(t, \boldsymbol{y}) & \cdots & \frac{\partial G}{\partial y_{d-1}}(t, \boldsymbol{y})
\end{array}\right] \\
& =\operatorname{det}\left[\begin{array}{llll}
\frac{d F_{t}}{d t}(\boldsymbol{y}) & \frac{\partial F_{t}}{\partial y_{1}}(\boldsymbol{y}) & \cdots & \frac{\partial F_{t}}{\partial y_{d-1}}(\boldsymbol{y})
\end{array}\right] \\
& =\operatorname{det}\left[\begin{array}{llll}
\frac{\mathbf{V}\left(F_{t}(\boldsymbol{y})\right)}{\phi\left(F_{t}(\boldsymbol{y})\right)} & \frac{\partial F_{t}}{\partial y_{1}}(\boldsymbol{y}) & \cdots & \frac{\partial F_{t}}{\partial y_{d-1}}(\boldsymbol{y})
\end{array}\right] .
\end{aligned}
$$

For a fixed $\boldsymbol{y} \in \sigma \backslash Z_{\sigma}$ and for small $t$ the flow $F_{t}(\boldsymbol{y})$ occurs in a region where $\mathbf{V}$ and $\phi$ (and thus $F_{t}$ ) are smooth - namely, the side of $\sigma$ determined by the sign of $\mathbf{V}(\boldsymbol{y}) \cdot \mathbf{n}_{\sigma}$. Hence, since $F_{0}=\mathrm{Id}$, denoting by $\left(\mathbf{V}_{1}, \ldots, \mathbf{V}_{d}\right)$ the components of $\mathbf{V}$ in the chosen basis and recalling that $\mathbf{n}_{\sigma}=(1,0, \ldots, 0)$,

$$
\lim _{t \rightarrow 0} J G(t, \boldsymbol{y})=\operatorname{det}\left[\begin{array}{llll}
\frac{\mathbf{V}(\boldsymbol{y})}{\phi(\boldsymbol{y})} & \frac{\partial F_{0}}{\partial y_{1}}(\boldsymbol{y}) & \cdots & \frac{\partial F_{0}}{\partial y_{d-1}}(\boldsymbol{y})
\end{array}\right]
$$




$$
=\operatorname{det}\left[\begin{array}{ccccc}
\frac{\mathbf{V}_{1}(\boldsymbol{y})}{\phi(\boldsymbol{y})} & 0 & \cdots & \cdots & 0 \\
\vdots & 1 & 0 & \cdots & 0 \\
\vdots & 0 & \ddots & \ddots & \vdots \\
\vdots & \vdots & \ddots & \ddots & 0 \\
\frac{\mathbf{V}_{d}(\boldsymbol{y})}{\phi(\boldsymbol{y})} & 0 & \cdots & 0 & 1
\end{array}\right]=\frac{\mathbf{V}_{1}(\boldsymbol{y})}{\phi(\boldsymbol{y})}=\frac{\mathbf{V}(\boldsymbol{y}) \cdot \mathbf{n}_{\sigma}}{\phi(\boldsymbol{y})}
$$

Here, the value of $\phi$ is of course considered on the side of $\sigma$ into which $F_{t}(\boldsymbol{y})$ flows for small $t>0$ (as already noticed, the value of $\mathbf{V}(\boldsymbol{y}) \cdot \mathbf{n}_{\sigma}$ does not depend on the considered side). Recalling that (31) holds for $\boldsymbol{y} \in \sigma \backslash Z_{\sigma}$ and that $Z_{\sigma}$ has zero $(d-1)$-dimensional measure, the dominated convergence theorem thus shows that

$$
\int_{\sigma}|J G(t, \boldsymbol{y})| d \mathrm{~s}(\boldsymbol{y}) \rightarrow \int_{\sigma} \frac{\left|\mathbf{V}(\boldsymbol{y}) \cdot \mathbf{n}_{\sigma}\right|}{\phi(\boldsymbol{y})} d \mathrm{~s}(\boldsymbol{y}) \text { as } t \rightarrow 0 .
$$

Dividing (30) by $h$, letting $h \rightarrow 0$, and plugging the result in (29) we infer that

$$
\frac{d V_{t}}{d t} \leq \frac{C_{1}(T)}{\phi_{*}} \int_{\sigma}\left|\mathbf{V}(\boldsymbol{y}) \cdot \mathbf{n}_{\sigma}\right| d \mathrm{~s}(\boldsymbol{y}) .
$$

The mapping $t \mapsto V_{t}$ is a non-decreasing function, so its derivative in the sense of distributions always exists as a positive measure; the relation above shows that this derivative is actually a bounded function, and thus that $t \mapsto V_{t}$ is Lipschitzcontinuous. Integrating this relation and using $V_{0}=0$ leads to (28).

We can now state a result that mimics Lemma 5.3 but for piecewise-constant functions. This result is used in Section 4.2.2 to prove that HMM schemes, among others, satisfy (A2).

Lemma 5.5 (Translation estimate for piecewise-constant functions). Let $\mathfrak{T}$ be a polytopal mesh and $Y_{\mathcal{M}}$ be the set of piecewise-constant functions on $\mathcal{M}$. Define the discrete $H^{1}$-semi norm on $Y_{\mathcal{M}}$ by

$$
\forall f \in Y_{\mathcal{M}},|f|_{\mathfrak{T}}=\left(\sum_{\sigma \in \mathcal{E}_{\text {int }}}|\sigma| d_{\sigma}\left|\frac{f_{K}-f_{L}}{d_{\sigma}}\right|^{2}\right)^{1 / 2},
$$

where $f_{K}$ is the constant value of $f$ on $K \in \mathcal{M}, \mathcal{E}_{\text {int }}$ is the set of internal faces (that is, $\sigma \in \mathcal{E}$ such that $\sigma \subset \Omega$ ), $K$ and $L$ are the two cells on each side of $\sigma$, and $d_{\sigma}=d_{K, \sigma}+d_{L, \sigma}$ (see Figure 1). Assume that $(\phi, \mathbf{V})$ satisfy (21) on the sub-mesh made of $\left(D_{K, \sigma}\right)_{K \in \mathcal{M}, \sigma \in \mathcal{E}_{K}}$ and let $k$ be the maximal polynomial degree of $\mathbf{V}$.

Then, if $\varrho \geq \varrho \mathfrak{T}$ (defined by (14)), there exists $R$ depending only on $k, d$ and $\varrho$ such that, for all $s \in[-T, T]$,

$$
\forall f \in Y_{\mathcal{M}},\left\|f\left(F_{s}\right)-f\right\|_{L^{1}(\Omega)} \leq R \frac{C_{1}(T)}{\phi_{*}}|s|\|\mathbf{V}\|_{L^{2}(\Omega)}|f|_{\mathfrak{T}}
$$

where $C_{1}(T)=\frac{\phi^{*}}{\phi_{*}} \exp \left(\frac{\Gamma_{\text {div }} T}{\phi_{*}}\right)$ as in $(23)$.

Proof. We start by writing $f\left(F_{s}(\boldsymbol{x})\right)-f(\boldsymbol{x})$ as the sum of the jumps of $f$ along the curve $\left(F_{t}(\boldsymbol{x})\right)_{t \in[0, s]}=: F_{[0, s]}(\boldsymbol{x})$. For $\sigma \in \mathcal{E}_{\text {int }}$, letting $\chi_{\sigma}(\boldsymbol{x})=1$ if $\sigma \cap F_{[0, s]}(\boldsymbol{x}) \neq \emptyset$ and $\chi_{\sigma}(\boldsymbol{x})=0$ otherwise, this leads to

$$
\left|f\left(F_{s}(\boldsymbol{x})\right)-f(\boldsymbol{x})\right| \leq \sum_{\sigma \in \mathcal{E}_{\mathrm{int}}} \chi_{\sigma}(\boldsymbol{x})\left|f_{K}-f_{L}\right| .
$$


Notice that $\sigma \cap F_{[0, s]}(\boldsymbol{x}) \neq \emptyset$ if and only if $F_{[-s, 0]}(\sigma) \cap\{\boldsymbol{x}\} \neq \emptyset$, that is, $\boldsymbol{x}$ belongs to the region covered by $\sigma$ transported by the flow over $[-s, 0]$. Lemma 5.4 gives

$$
\int_{\Omega} \chi_{\sigma}(\boldsymbol{x}) d \boldsymbol{x} \leq \frac{C_{1}(T)}{\phi_{*}}|s| \int_{\sigma}\left|\mathbf{V}(\boldsymbol{y}) \cdot \mathbf{n}_{\sigma}\right| d \mathrm{~s}(\boldsymbol{y})
$$

where $\mathbf{n}_{\sigma}$ is a unit normal to $\sigma$. Hence, letting $C=\frac{C_{1}(T)}{\phi_{*}}$ and using the CauchySchwarz inequality (on the combined sum and integral terms),

$$
\begin{aligned}
& \int_{\Omega}\left|f\left(F_{s}(\boldsymbol{x})\right)-f(\boldsymbol{x})\right| d \boldsymbol{x} \\
& \leq C|s| \sum_{\sigma \in \mathcal{E}_{\mathrm{int}}} \int_{\sigma}\left|\mathbf{V}(\boldsymbol{y}) \cdot \mathbf{n}_{\sigma}\right|\left|f_{K}-f_{L}\right| d \mathrm{~s}(\boldsymbol{y}) \\
& =C|s| \sum_{\sigma \in \mathcal{E}_{\text {int }}} \int_{\sigma} \sqrt{d_{\sigma}}\left|\mathbf{V}(\boldsymbol{y}) \cdot \mathbf{n}_{\sigma}\right| \frac{1}{\sqrt{d_{\sigma}}}\left|f_{K}-f_{L}\right| d \mathrm{~s}(\boldsymbol{y}) \\
& \leq C|s|\left(\sum_{\sigma \in \mathcal{E}_{\text {int }}} \int_{\sigma} d_{\sigma}\left|\mathbf{V}(\boldsymbol{y}) \cdot \mathbf{n}_{\sigma}\right|^{2} d \mathrm{~s}(\boldsymbol{y})\right)^{1 / 2}\left(\sum_{\sigma \in \mathcal{E}_{\text {int }}} \int_{\sigma} \frac{1}{d_{\sigma}}\left|f_{K}-f_{L}\right|^{2} d \mathrm{~s}(\boldsymbol{y})\right)^{1 / 2} \\
& =C|s|\left(\sum_{\sigma \in \mathcal{E}_{\text {int }}} d_{\sigma} \int_{\sigma}\left|\mathbf{V}(\boldsymbol{y}) \cdot \mathbf{n}_{\sigma}\right|^{2} d \mathrm{~s}(\boldsymbol{y})\right)^{1 / 2}|f|_{\mathfrak{T}} .
\end{aligned}
$$

Since $\mathbf{V}$ is polynomial on each $D_{K, \sigma}$, we can use the discrete trace inequality of [13, Lemma 1.46] to find $R$ depending only on $k, d$ and $\varrho$ such that

$$
\forall K \in \mathcal{M}, \forall \sigma \in \mathcal{E}_{K}, \operatorname{diam}\left(D_{K, \sigma}\right) \int_{\sigma}\left|\mathbf{V}(\boldsymbol{y}) \cdot \mathbf{n}_{\sigma}\right|^{2} d \mathrm{~s}(\boldsymbol{y}) \leq R^{2} \int_{D_{K, \sigma}}|\mathbf{V}(\boldsymbol{x})|^{2} d \boldsymbol{x} .
$$

Noticing that $d_{K, \sigma} \leq \operatorname{diam}\left(D_{K, \sigma}\right)$, we infer

$$
d_{\sigma} \int_{\sigma}\left|\mathbf{V}(\boldsymbol{y}) \cdot \mathbf{n}_{\sigma}\right|^{2} d \mathrm{~s}(\boldsymbol{y}) \leq R^{2} \int_{D_{K, \sigma} \cup D_{L, \sigma}}|\mathbf{V}(\boldsymbol{x})|^{2} d \boldsymbol{x} .
$$

The proof of the lemma is completed by plugging this estimate into (34).

Remark 5.6 (Estimate in $L^{\alpha}$ norm?). A natural question would be the extension of Lemma 5.5 to estimate the $L^{\alpha}$ norm of $f\left(F_{s}\right)-f$, as in Lemma 5.3, by using the discrete $W^{1, r}$-semi norm $|f|_{\mathfrak{T}, r}$ of $f$ obtained by replacing 2 with $r$ in (32). Considering for example the simple case of a constant unit velocity $\mathbf{V}=\mathbf{V}_{0}$ (and forgetting about boundary conditions for simplification), this would amount to estimating $\left\|f\left(\cdot+s \mathbf{V}_{0}\right)-f\right\|_{L^{\alpha}(\Omega)}$ in terms of $|s||f|_{\mathfrak{T}, r}$. For meshes admissible for the TPFA finite volume scheme, such an estimate is known with $\alpha=r=2$ and $|s|$ replaced by $\sqrt{|s|\left(|s|+\max _{K \in \mathcal{M}} \operatorname{diam}(K)\right)}[21$, Lemma 3.3]. For general meshes, however, no similar estimate seems to be attainable if $\alpha>1$.

The next lemma is instrumental in passing to the limit in the reaction and advection terms of the GDM-ELLAM scheme. Let us first introduce some notations. Given time steps $0=t^{(0)}<t^{(1)}<\ldots<t^{(N)}=T$ and velocities $\mathbf{V}=\left(\mathbf{V}^{n}\right)_{n=1, \ldots, N}$ that satisfy (21), we identify $\mathbf{V}$ with the global velocity $\Omega \times(0, T) \rightarrow \mathbb{R}^{d}$ given by $\mathbf{V}(\cdot, t)=\mathbf{V}^{(n+1)}$ for all $t \in\left(t^{(n)}, t^{(n+1)}\right]$ and all $n=0, \ldots, N-1$. Define $\mathcal{T}_{\mathbf{V}}$ 
and $\widehat{\mathcal{T}}_{\mathbf{V}}$ as the linear mappings $L^{2}(\Omega \times(0, T)) \rightarrow L^{2}(\Omega \times(0, T))$ such that, for $\psi \in L^{2}(\Omega \times(0, T))$,

for a.e. $\boldsymbol{x} \in \Omega$, for all $t \in\left(t^{(n)}, t^{(n+1)}\right)$ and $n=0, \ldots, N-1$,

$$
\mathcal{T}_{\mathbf{V}} \psi(\boldsymbol{x}, t)=\psi\left(F_{t^{(n+1)}-t^{(n)}}^{(n+1)}(\boldsymbol{x}), t\right) \quad \text { and } \quad \widehat{\mathcal{T}}_{\mathbf{V}} \psi(\boldsymbol{x}, t)=\psi\left(F_{t^{(n+1)}-t}^{(n+1)}(\boldsymbol{x}), t\right)
$$

where $F_{t}^{(n+1)}$ is defined by (19) for the velocity $\mathbf{V}^{(n+1)}$. The difference between $\mathcal{T}_{\mathbf{V}}$ and $\widehat{\mathcal{T}}_{\mathbf{V}}$ is the time at which this flow is considered.

Lemma 5.7 (Convergence of functions transported by the flow). Let $\phi$ satisfy (2b) and, for each $m \in \mathbb{N}$, take $0=t_{m}^{(0)}<t_{m}^{(1)}<\ldots<t_{m}^{\left(N_{m}\right)}=T$ time steps and $\mathbf{V}_{m}=\left(\mathbf{V}_{m}^{n}\right)_{n=1, \ldots, N_{m}}$ that satisfy $(21)$ with $\Gamma_{\text {div }}$ not depending on $m$. Assume that $\delta t_{m}:=\max _{n=0, \ldots, N_{m}-1}\left(t_{m}^{(n+1)}-t_{m}^{(n)}\right) \rightarrow 0$ as $m \rightarrow \infty$ and that $\left(\mathbf{V}_{m}\right)_{m \in \mathbb{N}}$ is bounded in $L^{2}(\Omega \times(0, T))$. Then $\mathcal{T}_{\mathbf{V}_{m}}$ and $\widehat{\mathcal{T}}_{\mathbf{V}_{m}}$ satisfy the following properties.

(1) There is $C$ not depending on $m$ such that, for $\psi \in L^{2}(\Omega \times(0, T))$,

$$
\left\|\mathcal{T}_{\mathbf{V}_{m}} \psi\right\|_{L^{2}(\Omega \times(0, T))}+\left\|\widehat{\mathcal{T}}_{\mathbf{V}_{m}} \psi\right\|_{L^{2}(\Omega \times(0, T))} \leq C\|\psi\|_{L^{2}(\Omega \times(0, T))}
$$

(2) The dual operators $\mathcal{T}_{\mathbf{V}_{m}}^{*}$ and $\widehat{\mathcal{T}}_{\mathbf{V}_{m}}^{*}$ of $\mathcal{T}_{\mathbf{V}_{m}}$ and $\mathcal{T}_{\mathbf{V}_{m}}$ are given by: for $\psi \in$ $L^{2}(\Omega \times(0, T))$,

$$
\begin{aligned}
& \mathcal{T}_{\mathbf{V}_{m}}^{*} \psi=\phi \mathcal{T}_{-\mathbf{V}_{m}}\left(\frac{\psi}{\phi}\right)+R_{m} \mathcal{T}_{-\mathbf{V}_{m}} \psi \\
& \widehat{\mathcal{T}}_{\mathbf{V}_{m}}^{*} \psi=\phi \widehat{\mathcal{T}}_{-\mathbf{V}_{m}}\left(\frac{\psi}{\phi}\right)+\widehat{R}_{m} \widehat{\mathcal{T}}_{-\mathbf{V}_{m}} \psi
\end{aligned}
$$

where $R_{m}, \widehat{R}_{m} \in L^{\infty}(\Omega \times(0, T))$ and, over each interval $\left[t^{(n)}, t^{(n+1)}\right]$, $R_{m}, \widehat{R}_{m}$ are bounded by $\delta t^{\left(n+\frac{1}{2}\right)} \phi_{*}^{-1} \Gamma_{\mathrm{div}} C_{1}(T)$.

(3) If $f_{m} \rightarrow f$ strongly (resp. weakly) in $L^{2}(\Omega \times(0, T))$ as $m \rightarrow \infty$, then $\mathcal{T}_{\mathbf{V}_{m}} f_{m} \rightarrow f$ and $\widehat{\mathcal{T}}_{\mathbf{V}_{m}} f_{m} \rightarrow f$ strongly (resp. weakly) in $L^{2}(\Omega \times(0, T))$.

Proof.

We only prove the results for $\mathcal{T}_{\mathbf{V}_{m}}$, as the proof for $\widehat{\mathcal{T}}_{\mathbf{V}_{m}}$ follows by simply replacing $F_{t^{(n+1)}-t^{(n)}}^{(n+1)}(\boldsymbol{y})$ by $F_{t^{(n+1)}-t}^{(n+1)}(\boldsymbol{y})$. In the first two steps, we drop the index $m$ in $\mathbf{V}_{m}$ and $N_{m}$ for simplicity of notation.

Step 1: bound on the norms of $\mathcal{T}_{\mathbf{V}}$ and $\widehat{\mathcal{T}}_{\mathbf{V}}$.

By a change of variables and invoking (23), there is $C$ not depending on $m$, $s \in[-T, T]$ or $n \in\{0, \ldots, N-1\}$ such that, for all $h \in L^{2}(\Omega),\left\|h\left(F_{s}^{(n+1)}(\cdot)\right)\right\|_{L^{2}(\Omega)} \leq$ $C\|h\|_{L^{2}(\Omega)}$. Estimate (36) easily follows from this.

Step 2: description of the dual operator.

A change of variables yields, for any $\varphi, \psi \in L^{2}(\Omega \times(0, T))$,

$$
\begin{aligned}
& \int_{\Omega \times(0, T)}\left(\mathcal{T}_{\mathbf{V}} \varphi\right)(\boldsymbol{x}, t) \psi(\boldsymbol{x}, t) d \boldsymbol{x} d t \\
& =\sum_{n=0}^{N-1} \int_{t^{(n)}}^{t^{(n+1)}} \int_{\Omega} \varphi\left(F_{t^{(n+1)}-t^{(n)}}^{(n+1)}(\boldsymbol{x}), t\right) \psi(\boldsymbol{x}, t) d \boldsymbol{x} d t \\
& =\sum_{n=0}^{N-1} \int_{t^{(n)}}^{t^{(n+1)}} \int_{\Omega} \varphi(\boldsymbol{y}, t) \psi\left(F_{t^{(n)}-t^{(n+1)}}^{(n+1)}(\boldsymbol{y}), t\right)\left|J F_{t^{(n)}-t^{(n+1)}}^{(n+1)}(\boldsymbol{y})\right| d \boldsymbol{y} d t .
\end{aligned}
$$


Relation (22) and Estimate (23) shows that

$$
\left|J F_{t^{(n)}-t^{(n+1)}}^{(n+1)}(\boldsymbol{y})\right|=\frac{\phi(\boldsymbol{y})}{\phi\left(F_{t^{(n)}-t^{(n+1)}}^{(n+1)}(\boldsymbol{y})\right)}+R\left(\boldsymbol{y}, t^{(n)}\right)
$$

with $\left|R\left(\boldsymbol{y}, t^{(n)}\right)\right| \leq \delta t^{\left(n+\frac{1}{2}\right)} \phi_{*}^{-1} \Gamma_{\operatorname{div}} C_{1}(T)$. Since $t \mapsto F_{t-t^{(n+1)}}^{(n+1)}(\boldsymbol{y})$ is the flow corresponding to $-\mathbf{V}$, Relations (38) and (39) then yield (37) for $\mathcal{T}_{\mathbf{V}}^{*}$.

Step 3: proof of the strong convergence.

For simplicity of notation, denote $\|\cdot\|_{2}=\|\cdot\|_{L^{2}(\Omega \times(0, T))}$. Assume that $f_{m} \rightarrow f$ strongly in $L^{2}(\Omega \times(0, T))$, and let $f^{\varepsilon}$ be a smooth approximation of $f$ such that $\left\|f-f^{\varepsilon}\right\|_{2} \leq \varepsilon$. The triangle inequality and (36) yield

$$
\begin{aligned}
\left\|\mathcal{T}_{\mathbf{V}_{m}} f_{m}-f\right\|_{2} \leq & \left\|\mathcal{T}_{\mathbf{V}_{m}}\left(f_{m}-f\right)\right\|_{2}+\left\|\mathcal{T}_{\mathbf{V}_{m}}\left(f-f^{\varepsilon}\right)\right\|_{2}+\left\|\mathcal{T}_{\mathbf{V}_{m}} f^{\varepsilon}-f^{\varepsilon}\right\|_{2} \\
& +\left\|f^{\varepsilon}-f\right\|_{2} \\
\leq & C\left\|f_{m}-f\right\|_{2}+(C+1) \varepsilon+\left\|\mathcal{T}_{\mathbf{V}_{m}} f^{\varepsilon}-f^{\varepsilon}\right\|_{2} .
\end{aligned}
$$

Invoking Lemma 5.3 with $\alpha=2, r=\infty$ and $f^{\varepsilon}(\cdot, t)$ instead of $f$ gives gives $C^{\prime}$ not depending on $m$ or $\varepsilon$ such that, if $F_{m, t}^{(n+1)}$ is the flow for the velocity $\mathbf{V}_{m}^{(n+1)}$,

$$
\begin{aligned}
\left\|\mathcal{T}_{\mathbf{V}_{m}} f^{\varepsilon}-f^{\varepsilon}\right\|_{2}^{2} & =\sum_{n=0}^{N_{m}-1} \int_{t^{(n)}}^{t^{(n+1)}}\left\|f^{\varepsilon}\left(F_{m, t^{(n+1)}-t^{(n)}}^{(n+1)}(\cdot), t\right)-f^{\varepsilon}(\cdot, t)\right\|_{L^{2}(\Omega)}^{2} d t \\
& \leq C^{\prime} \delta t_{m}^{2} \sum_{n=0}^{N_{m}-1} \int_{t^{(n)}}^{t^{(n+1)}}\left\|\mathbf{V}_{m}^{(n+1)}\right\|_{L^{2}(\Omega)}^{2}\left\|\nabla f^{\varepsilon}(\cdot, t)\right\|_{L^{\infty}(\Omega)}^{2} d t \\
& =C^{\prime} \delta t_{m}^{2}\left\|\mathbf{V}_{m}\right\|_{2}^{2}\left\|\nabla f^{\varepsilon}\right\|_{L^{\infty}(\Omega \times(0, T))}^{2} .
\end{aligned}
$$

Hence,

$$
\left\|\mathcal{T}_{\mathbf{V}_{m}} f_{m}-f\right\|_{2} \leq C\left\|f_{m}-f\right\|_{2}+(1+C) \varepsilon+\sqrt{C^{\prime}} \delta t_{m}\left\|\mathbf{V}_{m}\right\|_{2}\left\|\nabla f^{\varepsilon}\right\|_{L^{\infty}(\Omega \times(0, T))} .
$$

Taking the superior limit as $m \rightarrow \infty$ and using the boundedness of $\left(\mathbf{V}_{m}\right)_{m \in \mathbb{N}}$ in $L^{2}(\Omega \times(0, T))$ thus yields $\lim \sup _{m \rightarrow \infty}\left\|\mathcal{T}_{\mathbf{V}_{m}} f_{m}-f\right\|_{2} \leq(1+C) \varepsilon$. Letting $\varepsilon \rightarrow 0$ concludes the proof that $\mathcal{T}_{\mathbf{V}_{m}} f_{m} \rightarrow f$ strongly in $L^{2}(\Omega \times(0, T))$.

Step 4: proof of the weak convergence.

Assume that $f_{m} \rightarrow f$ weakly in $L^{2}(\Omega \times(0, T))$. Then, for all $\psi \in L^{2}(\Omega \times(0, T))$,

$$
\begin{aligned}
\int_{\Omega \times(0, T)}\left(\mathcal{T}_{\mathbf{V}_{m}} f_{m}-f\right) \psi & =\int_{\Omega \times(0, T)} \mathcal{T}_{\mathbf{V}_{m}}\left(f_{m}-f\right) \psi+\int_{\Omega \times(0, T)}\left(\mathcal{T}_{\mathbf{V}_{m}} f-f\right) \psi \\
& =\int_{\Omega \times(0, T)}\left(f_{m}-f\right) \mathcal{T}_{\mathbf{V}_{m}}^{*} \psi+\int_{\Omega \times(0, T)}\left(\mathcal{T}_{\mathbf{V}_{m}} f-f\right) \psi
\end{aligned}
$$

Since $\psi / \phi \in L^{2}(\Omega \times(0, T))$, the formula (37), the fact that $R_{m} \rightarrow 0$ in $L^{\infty}(\Omega \times$ $(0, T)$ ), the estimate (36) and the result of Step 3 applied to $-\mathbf{V}_{m}$ instead of $\mathbf{V}_{m}$ show that $\mathcal{T}_{\mathbf{V}_{m}}^{*} \psi \rightarrow \psi$ strongly in $L^{2}(\Omega \times(0, T))$ as $m \rightarrow \infty$. Hence, the first term in the right-hand side of (40) tends to 0 since $f_{m}-f \rightarrow 0$ weakly $L^{2}(\Omega \times(0, T))$. The second term in the right-hand side of (40) also converges to 0 since, by Step 3 (applied to $f_{m}=f$ for all $\left.m\right), \mathcal{T}_{\mathbf{V}_{m}} f-f \rightarrow 0$ in $L^{2}(\Omega \times(0, T))$. The proof that $\mathcal{T}_{\mathbf{V}_{m}} f_{m} \rightarrow f$ weakly in $L^{2}(\Omega \times(0, T))$ is therefore complete. 


\section{A Priori Estimates}

Throughout this section, $A \lesssim B$ means that $A \leq C B$, where $C$ is a constant depending only on the quantities $|\Omega|, T, \phi_{*}, \phi^{*}, \alpha_{A}, \alpha_{\mathbf{D}}, \Lambda_{A}, \Lambda_{\mathbf{D}}, M_{q^{-}}, M_{q^{+}}, M_{t}$, $M_{F}, M_{\mathrm{div}}, \sup _{m \in \mathbb{N}} C_{\mathcal{P}_{m}}, \sup _{m \in \mathbb{N}} C_{\mathcal{C}_{m}}$ appearing in Assumptions (2) and (A1)(A5) $\left(C_{\mathcal{P}_{m}}\right.$ and $C_{\mathcal{C}_{m}}$ are given by (5)). Likewise, in the proofs, $C$ denotes a generic constant that can change from one line to the other, but only depends on the aforementioned parameters.

We also consider that $\left(p_{m}, c_{m}\right)$ is a solution to the GDM-ELLAM scheme with $\left(\mathcal{P}, \mathcal{C}^{T}\right)=\left(\mathcal{P}_{m}, \mathcal{C}_{m}^{T}\right)$ and we drop the index $m$ for legibility. Let $\mathbf{U}_{\mathcal{P}}=-\frac{\mathbf{K}}{\mu\left(\widetilde{\Pi}_{\mathcal{C}} c\right)} \nabla_{\mathcal{P}} p$.

Lemma 6.1 (Estimates on the pressure). The following estimate holds:

$$
\left\|\Pi_{\mathcal{P}} p\right\|_{L^{\infty}\left(0, T ; L^{2}(\Omega)\right)}+\left\|\nabla_{\mathcal{P}} p\right\|_{L^{\infty}\left(0, T ; L^{2}(\Omega)\right)}+\left\|\mathbf{U}_{\mathcal{P}}\right\|_{L^{\infty}\left(0, T ; L^{2}(\Omega)\right)} \lesssim 1 .
$$

Proof. Setting $z=p^{(n+1)}$ in the gradient scheme (6), we get:

$$
\int_{\Omega} A\left(\boldsymbol{x}, \Pi_{\mathcal{C}} c^{(n)}\right) \nabla_{\mathcal{P}} p^{(n+1)} \cdot \nabla_{\mathcal{P}} p^{(n+1)}=\int_{\Omega}\left(q_{n}^{+}-q_{n}^{-}\right) \Pi_{\mathcal{P}} p^{(n+1)} .
$$

Using (2c) for the left hand side, followed by Cauchy-Schwarz' inequality

$$
\left\|\nabla_{\mathcal{P}} p^{(n+1)}\right\|_{L^{2}(\Omega)}^{2} \lesssim\left\|q_{n}^{+}-q_{n}^{-}\right\|_{L^{2}(\Omega)}\left\|\Pi_{\mathcal{P}} p^{(n+1)}\right\|_{L^{2}(\Omega)} \lesssim\left\|\nabla_{\mathcal{P}} p^{(n+1)}\right\|_{L^{2}(\Omega)}
$$

where we used

$$
\left\|\Pi_{\mathcal{P}} p^{(n+1)}\right\|_{L^{2}(\Omega)} \lesssim\left\|p^{(n+1)}\right\|_{\mathcal{P}}=\left\|\nabla_{\mathcal{P}} p^{(n+1)}\right\|_{L^{2}(\Omega)}
$$

since $\int_{\Omega} \Pi_{\mathcal{P}} p^{(n+1)}=0$. Equation (41) proves the estimate on $\nabla_{\mathcal{P}} p$ which gives the bound on $\mathbf{U}_{\mathcal{P}}$ (owing to (2c)) and, using (42) once more, provides the estimate on $\Pi_{\mathcal{P}} p$.

Lemma 6.2 (Estimates on the concentration). The following estimate holds:

$$
\left\|\Pi_{\mathcal{C}} c\right\|_{L^{\infty}\left(0, T ; L^{2}(\Omega)\right)}+\left\|\left(1+\left|\mathbf{U}_{\mathcal{P}}\right|\right)^{1 / 2} \nabla_{\mathcal{C}} c\right\|_{L^{2}\left(0, T ; L^{2}(\Omega)\right)} \lesssim 1+\left\|\Pi_{\mathcal{C}} \mathcal{I}_{\mathcal{C}} c_{\mathrm{ini}}\right\|_{L^{2}(\Omega)} .
$$

As a consequence, $\left\|\nabla_{\mathcal{C}} c\right\|_{L^{2}\left(0, T ; L^{2}(\Omega)\right)} \lesssim 1+\left\|\Pi_{\mathcal{C}} \mathcal{I}_{\mathcal{C}} c_{\mathrm{ini}}\right\|_{L^{2}(\Omega)}$.

Proof. Denote $Y_{n}=\left\|\Pi_{\mathcal{C}} c^{(n)} \sqrt{\phi}\right\|_{L^{2}(\Omega)}$. The gradient scheme (8) with $z=c^{(n+1)}$ yields

$$
\begin{aligned}
Y_{n+1}^{2} & -\int_{\Omega} \phi \Pi_{\mathcal{C}} c^{(n)} v\left(t^{(n)}\right)+\delta t^{\left(n+\frac{1}{2}\right)} \int_{\Omega} D\left(\boldsymbol{x}, \mathbf{U}_{\mathcal{P}}^{(n+1)}\right) \nabla_{\mathcal{C}} c^{(n+1)} \cdot \nabla_{\mathcal{C}} c^{(n+1)} \\
& +w \delta t^{\left(n+\frac{1}{2}\right)} \int_{\Omega} \Pi_{\mathcal{C}} c^{(n)} v\left(t^{(n)}\right) q_{n}^{-}+(1-w) \delta t^{\left(n+\frac{1}{2}\right)} \int_{\Omega}\left(\Pi_{\mathcal{C}} c^{(n+1)}\right)^{2} q_{n+1}^{-} \\
& =w \delta t^{\left(n+\frac{1}{2}\right)} \int_{\Omega} q_{n}^{+} v\left(t^{(n)}\right)+(1-w) \delta t^{\left(n+\frac{1}{2}\right)} \int_{\Omega} q_{n+1}^{+} \Pi_{\mathcal{C}} c^{(n+1)}=: \Delta .
\end{aligned}
$$

Now, by Cauchy-Schwarz, recalling that $0 \leq w \leq 1$ and that $\left|q_{n}^{-} / \sqrt{\phi}\right| \leq M_{q^{-}} / \sqrt{\phi_{*}}$, and using the coercivity property of the diffusion tensor $\mathbf{D}$,

$$
\begin{aligned}
\Delta \geq & Y_{n+1}^{2}-Y_{n}\left\|v\left(t^{(n)}\right) \sqrt{\phi}\right\|_{L^{2}(\Omega)}+\alpha_{\mathbf{D}} \delta t^{\left(n+\frac{1}{2}\right)}\left\|\left(1+\left|\mathbf{U}_{\mathcal{P}}^{(n+1)}\right|\right)\left|\nabla_{\mathcal{C}} c^{(n+1)}\right|^{2}\right\|_{L^{1}(\Omega)} \\
& -\frac{M_{q^{-}}}{\sqrt{\phi_{*}}} \delta t^{\left(n+\frac{1}{2}\right)} Y_{n}\left\|v\left(t^{(n)}\right)\right\|_{L^{2}(\Omega)} .
\end{aligned}
$$


Consider the term $Y_{n}\left\|v\left(t^{(n)}\right) \sqrt{\phi}\right\|_{L^{2}(\Omega)}$ in the right hand side of the inequality. Estimate $(24)$ with $w(\boldsymbol{x}, t)=v(\boldsymbol{x}, t)^{2}$ and $s=\delta t^{\left(n+\frac{1}{2}\right)}$ (so that $v\left(t^{(n+1)}-s\right)=$ $\left.v\left(t^{(n)}\right)\right)$, followed by Young's inequality, give, for any $\varepsilon>0$,

$$
\begin{aligned}
Y_{n}\left\|v\left(t^{(n)}\right) \sqrt{\phi}\right\|_{L^{2}(\Omega)} & \leq Y_{n} Y_{n+1} \sqrt{1+C \delta t^{\left(n+\frac{1}{2}\right)}} \leq Y_{n} Y_{n+1}\left(1+C \delta t^{\left(n+\frac{1}{2}\right)}\right) \\
& \leq \frac{1}{2} Y_{n}^{2}+\frac{1}{2} Y_{n+1}^{2}+\frac{C^{2} \delta t^{\left(n+\frac{1}{2}\right)}}{2 \varepsilon} Y_{n}^{2}+\frac{\delta t^{\left(n+\frac{1}{2}\right)} \varepsilon}{2} Y_{n+1}^{2} .
\end{aligned}
$$

Using (25),

$$
Y_{n}\left\|v\left(t^{(n)}\right)\right\|_{L^{2}(\Omega)} \leq C Y_{n} Y_{n+1} \leq \frac{C^{2}}{2 \varepsilon} Y_{n}^{2}+\frac{\varepsilon}{2} Y_{n+1}^{2}
$$

Using (43) together with (44), we then have

$$
\begin{aligned}
\Delta \geq & Y_{n+1}^{2}-\left(\frac{1}{2} Y_{n}^{2}+\frac{1}{2} Y_{n+1}^{2}+\frac{C^{2} \delta t^{\left(n+\frac{1}{2}\right)}}{2 \varepsilon} Y_{n}^{2}+\frac{\delta t^{\left(n+\frac{1}{2}\right)} \varepsilon}{2} Y_{n+1}^{2}\right) \\
& +\alpha_{\mathbf{D}} \delta t^{\left(n+\frac{1}{2}\right)}\left\|\left(1+\left|\mathbf{U}_{\mathcal{P}}^{(n+1)}\right|\right)\left|\nabla_{\mathcal{C}} c^{(n+1)}\right|^{2}\right\|_{L^{1}(\Omega)} \\
& -\frac{M_{q^{-}}}{\sqrt{\phi_{*}}} \delta t^{\left(n+\frac{1}{2}\right)}\left(\frac{C^{2}}{2 \varepsilon} Y_{n}^{2}+\frac{\varepsilon}{2} Y_{n+1}^{2}\right),
\end{aligned}
$$

which implies that

$$
\begin{aligned}
\frac{1}{2} Y_{n+1}^{2}-\frac{1}{2} Y_{n}^{2}+\alpha_{\mathbf{D}} \delta t^{\left(n+\frac{1}{2}\right)}\left\|\left(1+\left|\mathbf{U}_{\mathcal{P}}^{(n+1)}\right|\right)\left|\nabla_{\mathcal{C}} c^{(n+1)}\right|^{2}\right\|_{L^{1}(\Omega)} & \\
& \lesssim \Delta+\frac{\delta t^{\left(n+\frac{1}{2}\right)}}{\varepsilon} Y_{n}^{2}+\varepsilon \delta t^{\left(n+\frac{1}{2}\right)} Y_{n+1}^{2} .
\end{aligned}
$$

Now, using the boundedness of $q^{+}$, Young's inequality, the fact that $w \in[0,1]$ and (25) with $w(\boldsymbol{x}, t)=v(\boldsymbol{x}, t)^{2}$ and $s=\delta t^{\left(n+\frac{1}{2}\right)}$,

$$
\begin{aligned}
\Delta & \lesssim \delta t^{\left(n+\frac{1}{2}\right)}\left(\left\|v\left(t^{(n)}\right)\right\|_{L^{2}(\Omega)}+\left\|\Pi_{\mathcal{C}} c^{(n+1)}\right\|_{L^{2}(\Omega)}\right) \\
& \lesssim \delta t^{\left(n+\frac{1}{2}\right)}\left[\frac{1}{\varepsilon}+\varepsilon\left\|v\left(t^{(n)}\right)\right\|_{L^{2}(\Omega)}^{2}+\varepsilon Y_{n+1}^{2}\right] \lesssim \frac{\delta t^{\left(n+\frac{1}{2}\right)}}{\varepsilon}+\delta t^{\left(n+\frac{1}{2}\right)} \varepsilon Y_{n+1}^{2}
\end{aligned}
$$

Combining with (45), we find

$$
\begin{aligned}
\frac{1}{2} Y_{n+1}^{2}-\frac{1}{2} Y_{n}^{2}+\alpha_{\mathbf{D}} \delta t^{\left(n+\frac{1}{2}\right)}\left\|\left(1+\left|\mathbf{U}_{\mathcal{P}}^{(n+1)}\right|\right)\left|\nabla_{\mathcal{C}} c^{(n+1)}\right|^{2}\right\|_{L^{1}(\Omega)} & \\
& \lesssim \frac{\delta t^{\left(n+\frac{1}{2}\right)}}{\varepsilon}+\frac{\delta t^{\left(n+\frac{1}{2}\right)}}{\varepsilon} Y_{n}^{2}+\varepsilon \delta t^{\left(n+\frac{1}{2}\right)} Y_{n+1}^{2},
\end{aligned}
$$


which, upon taking a telescoping sum, yields

$$
\begin{aligned}
\frac{1}{2} Y_{n+1}^{2} & -\frac{1}{2} Y_{0}^{2}+\alpha_{\mathbf{D}} \sum_{k=0}^{n} \delta t^{\left(k+\frac{1}{2}\right)}\left\|\left(1+\left|\mathbf{U}_{\mathcal{P}}^{(n+1)}\right|\right)\left|\nabla_{\mathcal{C}} c^{(n+1)}\right|^{2}\right\|_{L^{1}(\Omega)} \\
& \lesssim \frac{1}{\varepsilon} \sum_{k=0}^{n} \delta t^{\left(k+\frac{1}{2}\right)}+\frac{1}{\varepsilon} \sum_{k=0}^{n} \delta t^{\left(k+\frac{1}{2}\right)} Y_{k}^{2}+\varepsilon \sum_{k=1}^{n+1} \delta t^{\left(k-\frac{1}{2}\right)} Y_{k}^{2} \\
& \lesssim \frac{1}{\varepsilon} T+\frac{1}{\varepsilon} \delta t^{\left(\frac{1}{2}\right)} Y_{0}^{2}+\varepsilon \delta t^{\left(n+\frac{1}{2}\right)} Y_{n+1}^{2}+\left(\frac{1}{\varepsilon}+\varepsilon\right) \sum_{k=1}^{n}\left(\delta t^{\left(k+\frac{1}{2}\right)}+\delta t^{\left(k-\frac{1}{2}\right)}\right) Y_{k}^{2} .
\end{aligned}
$$

Denoting by $C$ the hidden multiplicative constant in the last $\lesssim$ above, choose $\varepsilon=1 /(4 C T)$ to absorb the term $\varepsilon \delta t^{\left(n+\frac{1}{2}\right)} Y_{n+1}^{2}$ in the left-hand side. Since $\varepsilon$ depends only on fixed quantities, we no longer make it explicit and it disappears into the $\lesssim$ symbols. Setting $\delta t^{\left(-\frac{1}{2}\right)}=0$ the term $\delta t^{\left(\frac{1}{2}\right)} Y_{0}^{2}$ can be integrated in the last sum and we find

$$
Y_{n+1}^{2}+\left\|\left(1+\left|\mathbf{U}_{\mathcal{P}}\right|\right)\left|\nabla_{\mathcal{C}}\right|^{2}\right\|_{L^{1}\left(\Omega \times\left(0, t^{(n+1)}\right)\right.} \lesssim 1+Y_{0}^{2}+\sum_{k=0}^{n}\left(\delta t^{\left(k+\frac{1}{2}\right)}+\delta t^{\left(k-\frac{1}{2}\right)}\right) Y_{k}^{2} .
$$

Dropping for a moment the second term in the left-hand side, and letting $C$ denote the hidden multiplicative constant in $\lesssim$, a discrete Gronwall's inequality $[26$, Section 5] yields, for any $n=0, \ldots, N-1$,

$$
Y_{n+1}^{2} \leq C\left(1+Y_{0}^{2}\right) \exp \left(\sum_{k=0}^{n} C\left(\delta t^{\left(k+\frac{1}{2}\right)}+\delta t^{\left(k-\frac{1}{2}\right)}\right)\right) \leq C\left(1+Y_{0}^{2}\right) \exp (2 C T) .
$$

By noticing that $Y_{0} \leq \sqrt{\phi^{*}}\left\|\Pi_{\mathcal{C}} c^{(0)}\right\|_{L^{2}(\Omega)}=\sqrt{\phi^{*}}\left\|\Pi_{\mathcal{C}} \mathcal{I}_{\mathcal{C}} c_{\text {ini }}\right\|_{L^{2}(\Omega)}$, this proves the estimate on $\left\|\Pi_{\mathcal{C}} c\right\|_{L^{\infty}\left(0, T ; L^{2}(\Omega)\right)}$. Plugging this estimate in (46) with $n=N-1$ yields the estimate on $\left\|\left(1+\left|\mathbf{U}_{\mathcal{P}}\right|\right)^{1 / 2} \nabla_{\mathcal{C}}\right\|_{L^{2}\left(0, T ; L^{2}(\Omega)\right)}$ which, in turn, trivially provides a bound on $\left\|\nabla_{\mathcal{C}} c\right\|_{L^{2}\left(0, T ; L^{2}(\Omega)\right)}$.

Remark 6.3 (Estimate of the advection-reaction terms). A formal integration-byparts shows that, if $\mathbf{u}$ satisfies (1a),

$$
\int_{\Omega} \operatorname{div}(c \mathbf{u}) c+\int_{\Omega} q^{-} c^{2}=\frac{1}{2} \int_{\Omega}\left(q^{+}+q^{-}\right) c^{2} \geq 0 .
$$

When using $c$ as a test function in the continuous equation, the advection and reaction terms thus combine to create a non-negative quantity that can simply be discarded from the estimates (which thus hold under very weak assumptions on $\left.q^{ \pm}\right)$. This can be reproduced at the discrete level for upwind discretisations $[8,10]$. However, the structure of the ELLAM discretisation does not seem to lend itself to such an easy estimate of the advection-reaction terms, which is why the proof of Lemma 6.2 is a bit technical, and requires the boundedness of $q^{ \pm}$(to bound the Jacobian of the changes of variables - note that we do not require a bound on $\mathbf{u}$ itself, though).

A crucial step in the convergence proof is to establish the strong compactness of $\Pi_{\mathcal{C}} c$. This is done by using a discrete version of the Aubin-Simon theorem. The gradient estimates in Lemma 6.2 provides the compactness in space, which must be complemented by some sort of boundedness (in a dual norm) of the discrete 
time-derivative of $c$. Establishing this boundedness is the purpose of the following lemma. A dual norm $\|\cdot\|_{\star, \phi, \mathcal{C}}$ is defined on $\Pi_{\mathcal{C}}\left(X_{\mathcal{C}}\right)$ the following way:

$$
\begin{aligned}
& \forall w \in \Pi_{\mathcal{C}}\left(X_{\mathcal{C}}\right) \\
& \|w\|_{\star, \phi, \mathcal{C}}:=\sup \left\{\int_{\Omega} \phi w \Pi_{\mathcal{C}} v: v \in X_{\mathcal{C}},\left\|\nabla_{\mathcal{C}} v\right\|_{L^{4}(\Omega)}+\left\|\Pi_{\mathcal{C}} v\right\|_{L^{\infty}(\Omega)}=1\right\} .
\end{aligned}
$$

It can easily be checked that this is indeed a norm (if $w \neq 0$, write $w=\Pi_{\mathcal{C}} z$, take $v=z / N$ where $N=\left\|\nabla_{\mathcal{C}} z\right\|_{L^{4}(\Omega)}+\left\|\Pi_{\mathcal{C}} z\right\|_{L^{\infty}(\Omega)}>0$, and notice that $\|w\|_{\star, \phi, \mathcal{C}} \geq$ $\left.\int_{\Omega} \phi w(\boldsymbol{x}) \Pi_{\mathcal{C}} v(\boldsymbol{x}) d \boldsymbol{x}=N^{-1}\|\sqrt{\phi} w\|_{L^{2}(\Omega)}^{2}\right)$.

Lemma 6.4. Defining the discrete time derivative of $c$ by

$$
\delta_{\mathcal{C}} c(t)=\frac{\Pi_{\mathcal{C}} c^{(n+1)}-\Pi_{\mathcal{C}} c^{(n)}}{\delta t^{\left(n+\frac{1}{2}\right)}} \text { for all } t \in\left(t^{(n)}, t^{(n+1)}\right) \text { and all } n=0, \ldots, N-1,
$$

we have

$$
\int_{0}^{T}\left\|\delta_{\mathcal{C}}\right\|_{\star, \phi, \mathcal{C}}^{2} d t \lesssim 1+\left\|\Pi_{\mathcal{C}} \mathcal{I}_{\mathcal{C}} c_{\mathrm{ini}}\right\|_{L^{\infty}(\Omega)}^{2} .
$$

Proof. Take $z \in X_{\mathcal{C}}$ arbitrary in (8). Subtract and add $\int_{\Omega} \phi \Pi_{\mathcal{C}} c^{(n)} \Pi_{\mathcal{C}} z$ to get

$$
\begin{aligned}
\int_{\Omega} \phi\left(\Pi_{\mathcal{C}} c^{(n+1)}-\Pi_{\mathcal{C}} c^{(n)}\right) \Pi_{\mathcal{C}} z \\
=-\int_{\Omega} \phi \Pi_{\mathcal{C}} c^{(n)}\left(\Pi_{\mathcal{C}} z-v\left(t^{(n)}\right)\right)-\delta t^{\left(n+\frac{1}{2}\right)} \int_{\Omega} D\left(\boldsymbol{x}, \mathbf{U}_{\mathcal{P}}^{(n+1)}\right) \nabla_{\mathcal{C}} c^{(n+1)} \cdot \nabla_{\mathcal{C}} z \\
\quad-w \delta t^{\left(n+\frac{1}{2}\right)} \int_{\Omega} \Pi_{\mathcal{C}} c^{(n)} v\left(t^{(n)}\right) q_{n}^{-}-(1-w) \delta t^{\left(n+\frac{1}{2}\right)} \int_{\Omega} \Pi_{\mathcal{C}} c^{(n+1)} \Pi_{\mathcal{C}} z q_{n+1}^{-} \\
\quad+w \delta t^{\left(n+\frac{1}{2}\right)} \int_{\Omega} q_{n}^{+} v\left(t^{(n)}\right)+(1-w) \delta t^{\left(n+\frac{1}{2}\right)} \int_{\Omega} q_{n+1}^{+} \Pi_{\mathcal{C}} z .
\end{aligned}
$$

The terms on the right hand side of the equation are referred to as $T_{1}, T_{2}, \ldots, T_{6}$, respectively. For the term $T_{1}$, recall that $v\left(\boldsymbol{x}, t^{(n)}\right)=\Pi_{\mathcal{C}} z\left(F_{\delta t}(n+1 / 2)(\boldsymbol{x})\right)$. If $n=0$, recalling that $c^{(0)}=\mathcal{I}_{\mathcal{C}} c_{\text {ini }}$ and applying $(\mathbf{A 2})$ shows that

$$
\begin{aligned}
\left|T_{1}\right| & \lesssim\left\|\Pi_{\mathcal{C}} \mathcal{I}_{\mathcal{C}} c_{\mathrm{ini}}\right\|_{L^{\infty}(\Omega)}\left\|\Pi_{\mathcal{C}} z-\Pi_{\mathcal{C}} z\left(F_{\delta t^{(1 / 2)}}\right)\right\|_{L^{1}(\Omega)} \\
& \lesssim \delta t^{\left(\frac{1}{2}\right)}\left\|\Pi_{\mathcal{C}} \mathcal{I}_{\mathcal{C}} c_{\mathrm{ini}}\right\|_{L^{\infty}(\Omega)}\left\|\mathbf{u}_{\mathcal{P}}^{(1)}\right\|_{L^{2}(\Omega)}\left\|\nabla_{\mathcal{C}} z\right\|_{L^{2}(\Omega)} .
\end{aligned}
$$

If $n \neq 0$, a change of variables yields

$$
\begin{aligned}
&-T_{1}= \int_{\Omega} \phi \Pi_{\mathcal{C}} c^{(n)} \Pi_{\mathcal{C}} z \\
&-\int_{\Omega} \phi\left(F_{-\delta t^{(n+1 / 2)}}(\boldsymbol{x})\right) \Pi_{\mathcal{C}} c^{(n)}\left(F_{-\delta t}(n+1 / 2)\right. \\
&(\boldsymbol{x})) \Pi_{\mathcal{C}} z(\boldsymbol{x})\left|J F_{-\delta t^{(n+1 / 2)}}(\boldsymbol{x})\right| d \boldsymbol{x} .
\end{aligned}
$$

Applying (22) with $s=-\delta t^{\left(n+\frac{1}{2}\right)}$, we can thus write $-T_{1}=T_{11}-T_{12}$ with

$$
\begin{aligned}
& T_{11}=\int_{\Omega} \phi \Pi_{\mathcal{C}} c^{(n)} \Pi_{\mathcal{C}} z-\int_{\Omega} \phi(\boldsymbol{x}) \Pi_{\mathcal{C}} c^{(n)}\left(F_{-\delta t}^{(n+1 / 2)}(\boldsymbol{x})\right) \Pi_{\mathcal{C}} z(\boldsymbol{x}) d \boldsymbol{x} \\
& T_{12}=\int_{\Omega}\left[\Pi_{\mathcal{C}} c^{(n)}\left(F_{-\delta t}{ }^{(n+1 / 2)}(\boldsymbol{x})\right) \Pi_{\mathcal{C}} z(\boldsymbol{x})\right. \\
& \left.\times \int_{0}^{-\delta t^{\left(n+\frac{1}{2}\right)}}\left|J F_{t}(\boldsymbol{x})\right|\left(\operatorname{div} \mathbf{u}_{\mathcal{P}}^{(n+1)}\right) \circ F_{t}(\boldsymbol{x}) d t\right] d \boldsymbol{x} .
\end{aligned}
$$


Using (A2) leads to

$$
\begin{aligned}
\left|T_{11}\right| & \leq \int_{\Omega}\left|\phi \Pi_{\mathcal{C}} z\left(\Pi_{\mathcal{C}} c^{(n)}-\Pi_{\mathcal{C}} c^{(n)}\left(F_{-\delta t}(n+1 / 2)\right)\right)\right| \\
& \lesssim \delta t^{\left(n+\frac{1}{2}\right)}\left\|\Pi_{\mathcal{C}} z\right\|_{L^{\infty}(\Omega)}\left\|\mathbf{u}_{\mathcal{P}}^{(n+1)}\right\|_{L^{2}(\Omega)}\left\|\nabla_{\mathcal{D}} c^{(n)}\right\|_{L^{2}(\Omega)} .
\end{aligned}
$$

The boundedness of $\operatorname{div} \mathbf{u}_{\mathcal{P}}^{(n+1)}$ in (A4) and of $\left|J F_{t}\right|$ (see (23)) yield, by a change of variables,

$$
\begin{aligned}
&\left|T_{12}\right| \lesssim \delta t^{\left(n+\frac{1}{2}\right)} \| \Pi_{\mathcal{C}} c^{(n)}\left(F_{-\delta t}(n+1 / 2)\right) \\
& \\
& \lesssim \delta t^{\left(n+\frac{1}{2}\right)}\left\|\Pi_{L^{2}(\Omega)} c^{(n)}\right\|\left\|_{L^{2}(\Omega)}\right\| \Pi_{\mathcal{C}} z \|_{L^{2}(\Omega)} .
\end{aligned}
$$

For the term $T_{2}$, the property (2d) of the diffusion tensor $\mathbf{D}$ and Hölder's inequality with exponents 4,2 and 4 give

$$
\begin{aligned}
\left|T_{2}\right| & \lesssim \delta t^{\left(n+\frac{1}{2}\right)} \int_{\Omega} \sqrt{1+\left|\mathbf{U}_{\mathcal{P}}^{(n+1)}\right|}\left(\sqrt{1+\left|\mathbf{U}_{\mathcal{P}}^{(n+1)}\right|}\left|\nabla_{\mathcal{C}} c^{(n+1)}\right|\right)\left|\nabla_{\mathcal{C}} z\right| \\
& \lesssim \delta t^{\left(n+\frac{1}{2}\right)}\left\|1+\left|\mathbf{U}_{\mathcal{P}}^{(n+1)}\right|\right\|_{L^{2}(\Omega)}^{\frac{1}{2}}\left\|\left(1+\left|\mathbf{U}_{\mathcal{P}}^{(n+1)}\right|\right)^{\frac{1}{2}}\left|\nabla_{\mathcal{C}} c^{(n+1)}\right|\right\|_{L^{2}(\Omega)}\left\|\nabla_{\mathcal{C}} z\right\|_{L^{4}(\Omega)} .
\end{aligned}
$$

The terms $T_{3}$ to $T_{6}$ are estimated by using the Cauchy-Schwarz inequality:

$$
\begin{aligned}
\left|T_{3}\right| & \lesssim \delta t^{\left(n+\frac{1}{2}\right)}\left\|\Pi_{\mathcal{C}} c^{(n)}\right\|_{L^{2}(\Omega)}\left\|v\left(t^{(n)}\right)\right\|_{L^{2}(\Omega)}, \\
\left|T_{4}\right| & \lesssim \delta t^{\left(n+\frac{1}{2}\right)}\left\|\Pi_{\mathcal{C}} c^{(n+1)}\right\|_{L^{2}(\Omega)}\left\|\Pi_{\mathcal{C}} z\right\|_{L^{2}(\Omega)}, \\
\left|T_{5}+T_{6}\right| & \lesssim \delta t^{\left(n+\frac{1}{2}\right)}\left\|v\left(t^{(n)}\right)\right\|_{L^{2}(\Omega)}+\delta t^{\left(n+\frac{1}{2}\right)}\left\|\Pi_{\mathcal{C}} z\right\|_{L^{2}(\Omega)} \lesssim \delta t^{\left(n+\frac{1}{2}\right)}\left\|\Pi_{\mathcal{C}} z\right\|_{L^{2}(\Omega)}
\end{aligned}
$$

(we used (25) with $w=v^{2}$ and $s=\delta t^{\left(n+\frac{1}{2}\right)}$ to obtain $\left\|v\left(t^{(n)}\right)\right\|_{L^{2}(\Omega)} \lesssim\left\|\Pi_{\mathcal{C}} z\right\|_{L^{2}(\Omega)}$ ). For $n \neq 0$, combining the estimates from $T_{1}$ to $T_{6}$ leads to

$$
\begin{aligned}
& \int_{\Omega} \phi\left(\Pi_{\mathcal{C}} c^{(n+1)}-\Pi_{\mathcal{C}} c^{(n)}\right) \Pi_{\mathcal{C}} z \\
& \lesssim \delta t^{\left(n+\frac{1}{2}\right)}\left\|\Pi_{\mathcal{C}} z\right\|_{L^{\infty}(\Omega)}\left\|\mathbf{u}_{\mathcal{P}}^{(n+1)}\right\|_{L^{2}(\Omega)}\left\|\nabla_{\mathcal{C} c^{(n)}}\right\|_{L^{2}(\Omega)} \\
& \quad+\delta t^{\left(n+\frac{1}{2}\right)}\left\|\Pi_{\mathcal{C} c^{(n)}}\right\|_{L^{2}(\Omega)}\left\|\Pi_{\mathcal{C}} z\right\|_{L^{2}(\Omega)} \\
& \quad+\delta t^{\left(n+\frac{1}{2}\right)}\left\|1+\left|\mathbf{U}_{\mathcal{P}}^{(n+1)}\right|\right\|_{L^{2}(\Omega)}^{\frac{1}{2}}\left\|\left(1+\left|\mathbf{U}_{\mathcal{P}}^{(n+1)}\right|\right)^{\frac{1}{2}}\left|\nabla_{\mathcal{C}} c^{(n+1)}\right|\right\|_{L^{2}(\Omega)}\left\|\nabla_{\mathcal{C}} z\right\|_{L^{4}(\Omega)} \\
& \quad+\delta t^{\left(n+\frac{1}{2}\right)}\left\|\Pi_{\mathcal{C} c^{(n+1)}}\right\|_{L^{2}(\Omega)}\left\|\Pi_{\mathcal{C}} z\right\|_{L^{2}(\Omega)}+\delta t^{\left(n+\frac{1}{2}\right)}\left\|\Pi_{\mathcal{C}} z\right\|_{L^{2}(\Omega)} .
\end{aligned}
$$

Divide both sides by $\delta t^{\left(n+\frac{1}{2}\right)}$ and take the supremum over all $z \in X_{\mathcal{C}}$ with $\left\|\nabla_{\mathcal{C}} z\right\|_{L^{4}(\Omega)}+$ $\left\|\Pi_{\mathcal{C}} z\right\|_{L^{\infty}(\Omega)}=1$ to obtain, for all $n=1, \ldots, N-1$ and $t \in\left(t^{(n)}, t^{(n+1)}\right)$,

$$
\begin{aligned}
\left\|\delta_{\mathcal{C}} c(t)\right\|_{\star, \phi, \mathcal{C}} \lesssim & \left\|\mathbf{u}_{\mathcal{P}}^{(n+1)}\right\|_{L^{2}(\Omega)}\left\|\nabla_{\mathcal{C}} c^{(n)}\right\|_{L^{2}(\Omega)}+\left\|\Pi_{\mathcal{C}} c^{(n)}\right\|_{L^{2}(\Omega)}+\left\|\Pi_{\mathcal{C}} c^{(n+1)}\right\|_{L^{2}(\Omega)} \\
& +\left\|1+\left|\mathbf{U}_{\mathcal{P}}^{(n+1)}\right|\right\|_{L^{2}(\Omega)}^{\frac{1}{2}}\left\|\left(1+\left|\mathbf{U}_{\mathcal{P}}^{(n+1)}\right|\right)^{\frac{1}{2}} \nabla_{\mathcal{C}} c^{(n+1)}\right\|_{L^{2}(\Omega)}+1
\end{aligned}
$$


Square this, integrate for $t \in\left(t^{(n)}, t^{(n+1)}\right)$ and sum over $n=1, \ldots, N-1$. The assumption on the time steps in (A1) ensures that

$$
\begin{aligned}
\sum_{n=1}^{N-1} \delta t^{(n+1 / 2)}\left\|\nabla_{\mathcal{D}} c^{(n)}\right\|_{L^{2}(\Omega)}^{2} & \lesssim \sum_{n=1}^{N-1} \delta t^{(n-1 / 2)}\left\|\nabla_{\mathcal{D}} c^{(n)}\right\|_{L^{2}(\Omega)}^{2} \\
= & \sum_{n=0}^{N-2} \delta t^{(n+1 / 2)}\left\|\nabla_{\mathcal{D}} c^{(n+1)}\right\|_{L^{2}(\Omega)}^{2} \leq\left\|\nabla_{\mathcal{C}} c\right\|_{L^{2}(\Omega \times(0, T))}^{2}
\end{aligned}
$$

(and similarly for the terms involving $\Pi_{\mathcal{C}} c^{(n)}$ ), so that

$$
\begin{aligned}
& \int_{t^{(1)}}^{T}\left\|\delta_{\mathcal{C}} c(t)\right\|_{\star, \phi, \mathcal{C}}^{2} d t \lesssim\left\|\mathbf{u}_{\mathcal{P}}\right\|_{L^{\infty}\left(0, T ; L^{2}(\Omega)\right)}^{2}\left\|\nabla_{\mathcal{C}} c\right\|_{L^{2}(\Omega \times(0, T))}^{2}+\left\|\Pi_{\mathcal{C}} c\right\|_{L^{2}(\Omega \times(0, T))}^{2} \\
&+\left\|1+\left|\mathbf{U}_{\mathcal{P}}\right|\right\|_{L^{\infty}\left(0, T ; L^{2}(\Omega)\right)}\left\|\left(1+\left|\mathbf{U}_{\mathcal{P}}\right|\right)^{\frac{1}{2}} \nabla_{\mathcal{C}} c\right\|_{L^{2}(\Omega \times(0, T))}^{2}+1
\end{aligned}
$$

To estimate $\int_{0}^{t^{(1)}}\left\|\delta_{\mathcal{C}} c(t)\right\|_{\star, \phi, \mathcal{C}}^{2} d t$, we come back to (48) with $n=0$. The first term in the right-hand side of this inequality must be replaced by the right-hand side of (47), and thus the first term in (49) is replaced by $\left\|\Pi_{\mathcal{C}} \mathcal{I}_{\mathcal{C}} c_{\text {ini }}\right\|_{L^{\infty}(\Omega)}\left\|\mathbf{u}_{\mathcal{P}}^{(1)}\right\|_{L^{2}(\Omega)}$. Hence,

$$
\begin{aligned}
\int_{0}^{t^{(1)}}\left\|\delta_{\mathcal{C}} c(t)\right\|_{\star, \phi, \mathcal{C}}^{2} d t \lesssim \delta t^{(1 / 2)}\left\|\Pi_{\mathcal{C}} \mathcal{I}_{\mathcal{C}} c_{\mathrm{ini}}\right\|_{L^{\infty}(\Omega)}^{2}\left\|\mathbf{u}_{\mathcal{P}}^{(1)}\right\|_{L^{2}(\Omega)}^{2} \\
+\delta t^{(1 / 2)}\left\|\Pi_{\mathcal{C}} \mathcal{I}_{\mathcal{C}} c_{\mathrm{ini}}\right\|_{L^{2}(\Omega)}^{2}+\left\|\Pi_{\mathcal{C} c}\right\|_{L^{2}(\Omega \times(0, T))}^{2} \\
+\left\|1+\left|\mathbf{U}_{\mathcal{P}}\right|\right\|_{L^{\infty}\left(0, T ; L^{2}(\Omega)\right)}\left\|\left(1+\left|\mathbf{U}_{\mathcal{P}}\right|\right)^{\frac{1}{2}} \nabla_{\mathcal{C} c}\right\|_{L^{2}(\Omega \times(0, T))}^{2}+1 .
\end{aligned}
$$

The reason for separating the case $n \neq 0$ from the case $n=0$ is that, for $n=0$, (48) involves $\nabla_{\mathcal{C}} c^{(0)}=\nabla_{\mathcal{C}} \mathcal{I}_{\mathcal{C}} c_{\text {ini }}$ on which no bound has been imposed. The proof is completed by adding together (50) and (51), and by invoking Assumption (A5) and Lemmas 6.1 and 6.2.

\section{Proof of the main theorem}

At each time step, (6) and (8) are square linear equations on $p^{(n+1)}$ and $c^{(n+1)}$. The estimates of Lemma 6.1 and 6.2, together with the definition of the norms in $X_{\mathcal{P}}$ and $X_{\mathcal{C}}$, show that any solutions to these linear systems remains bounded. Hence, the matrices associated with these systems do not have any kernel, which ensures the existence and uniqueness of $(p, c)$ solution to the GDM-ELLAM scheme.

We now establish the compactness of $\left(\Pi_{\mathcal{C}_{m}} c_{m}\right)_{m \in \mathbb{N}}$, which is essential to proving the convergence of the pressure. Once this latter is establish, we conclude the proof by dealing with the convergence of the concentration.

\subsection{Compactness and initial convergence of $\Pi_{\mathcal{D}_{m}} c_{m}$.}

Theorem 7.1. Under the assumptions and notations of Theorem 3.3, the sequence $\left(\Pi_{\mathcal{C}_{m}} c_{m}\right)_{m \in \mathbb{N}}$ is relatively compact in $L^{2}\left(0, T ; L^{2}(\Omega)\right)$.

Proof. The idea is to apply Theorem 9.3 with $X_{m}=\Pi_{\mathcal{C}_{m}}\left(X_{\mathcal{C}_{m}}\right)$ equipped with the norm $\|u\|_{X_{m}}=\min \left\{\|w\|_{\mathcal{C}_{m}}: w \in X_{\mathcal{C}_{m}}\right.$ s.t. $\left.\Pi_{\mathcal{C}_{m}} w=u\right\}$ and $Y_{m}=X_{m}$ with the norm $\|\cdot\|_{Y_{m}}=\|\cdot\|_{\star, \phi, \mathcal{C}_{m}}$. 
Let us show that $\left(X_{m}, Y_{m}\right)_{m \in \mathbb{N}}$ is compactly-continuously embedded in $L^{2}(\Omega)$ (Definition 9.2). Item 1 follows by the compactness of $\left(\mathcal{C}_{m}\right)_{m \in \mathbb{N}}$, see Definition 2.2. Take now $\left(u_{m}\right)_{m \in \mathbb{N}}$ as prescribed in Item 2 and let $u$ be the limit in $L^{2}(\Omega)$ of this sequence. Let $\varphi \in C_{c}^{\infty}(\Omega)$ and consider the interpolant $\mathcal{J}_{\mathcal{C}_{m}}$ given by Assumption (A3). Then $\left\|\Pi_{\mathcal{C}_{m}} \mathcal{J}_{\mathcal{C}_{m}} \varphi\right\|_{L^{\infty}(\Omega)}+\left\|\nabla_{\mathcal{C}_{m}} \mathcal{J}_{\mathcal{C}_{m}} \varphi\right\|_{L^{4}(\Omega)} \leq C_{\varphi}$ for some $C_{\varphi}>0$ not depending on $m$, and thus, by definition of $\|\cdot\|_{Y_{m}}=\|\cdot\|_{\star, \phi, \mathcal{C}_{m}}$,

$$
\left|\int_{\Omega} \phi u_{m} \frac{\Pi_{\mathcal{C}_{m}} \mathcal{J}_{\mathcal{C}_{m}} \varphi}{C_{\varphi}}\right| \leq\left\|u_{m}\right\|_{Y_{m}} .
$$

Taking the limit as $m \rightarrow \infty$, we get $\int_{\Omega} \phi u \varphi=0$. Since this is true for all $\varphi \in C_{c}^{\infty}(\Omega)$, we deduce that $u=0$ as required.

We are left to show that the sequence $f_{m}=\left(\Pi_{\mathcal{C}_{m}} c_{m}\right)_{m \in \mathbb{N}}$ satisfies the properties in Theorem 9.3. The first property is trivially satisfied by the definition $f_{m}$, whereas the second and third one follow from Lemma 6.2 and the definition of the norm $\|\cdot\|_{\mathcal{C}_{m}}$ (Definition 2.1). The last property holds due to Lemma 6.4.

Thus, we may use Theorem 9.3 to conclude that the sequence $\left(\Pi_{\mathcal{C}_{m}} c_{m}\right)_{m \in \mathbb{N}}$ is relatively compact in $L^{2}\left(0, T ; L^{2}(\Omega)\right)$.

Theorem 7.1 together with Lemma 9.1 give $c \in L^{2}\left(0, T ; H^{1}(\Omega)\right)$ such that, up to a subsequence as $m \rightarrow \infty, \Pi_{\mathcal{C}_{m}} c_{m} \rightarrow c$ strongly in $L^{2}((0, T) \times \Omega)$ and $\nabla_{\mathcal{C}_{m}} c_{m} \rightarrow \nabla c$ weakly in $L^{2}((0, T) \times \Omega)^{d}$. From here on we always consider subsequences that satisfy these convergences. Let $\alpha_{m}:[0, T] \rightarrow \mathbb{R}$ be the piecewise affine map that maps each interval $\left(t_{m}^{(n)}, t_{m}^{(n+1)}\right)$ onto $\left(t_{m}^{(n-1)}, t_{m}^{(n)}\right)$, for $n=1, \ldots, N_{m}-1$. That is, $\alpha_{m}(t)=t-\left(1-\frac{\delta t_{m}^{(n-1 / 2)}}{\delta t_{m}^{(n+1 / 2)}}\right)\left(t-t^{(n)}\right)-\left(t^{(n)}-t^{(n-1)}\right)$ for $t \in\left(t^{(n)}, t^{(n+1)}\right)$. Recalling the definition of $\widetilde{\Pi}_{\mathcal{C}_{m}} c_{m}$ at the start of Section 6 , we have $\widetilde{\Pi}_{\mathcal{C}_{m}} c_{m}=\Pi_{\mathcal{C}_{m}} c_{m}\left(\cdot, \alpha_{m}(\cdot)\right)$ on $\Omega \times\left(t^{(1)}, T\right)$ and $\widetilde{\Pi}_{\mathcal{C}_{m}} c_{m}=\Pi_{\mathcal{C}_{m}} \mathcal{I}_{\mathcal{C}_{m}} c_{\text {ini }}$ on $\Omega \times\left(0, t^{(1)}\right)$. We have $\alpha_{m}(t) \rightarrow t$ uniformly as $m \rightarrow \infty$ and, due to (A1), the derivative of the inverse function $\alpha_{m}^{-1}$ is uniformly bounded. Hence, a triangle inequality, a change of variables using $\alpha_{m}^{-1}$, and the strong convergence of $\left(\Pi_{\mathcal{C}_{m}} c_{m}\right)_{m \in \mathbb{N}}$ show that $\widetilde{\Pi}_{\mathcal{C}_{m}} c_{m} \rightarrow c$ in $L^{2}(\Omega \times(0, T))$ as $m \rightarrow \infty$.

\subsection{Convergence of the pressure.}

Step 1: weak convergences of $\Pi_{\mathcal{P}_{m}} p_{m}$ and $\nabla_{\mathcal{P}_{m}} p_{m}$. We use Lemmas 6.1 and 9.1 to obtain $p \in L^{\infty}\left(0, T ; H^{1}(\Omega)\right)$ such that, up to a subsequence

$$
\begin{aligned}
& \Pi_{\mathcal{P}_{m}} p_{m} \rightarrow p \quad \text { weakly-* in } L^{\infty}\left(0, T ; L^{2}(\Omega)\right) \\
& \nabla_{\mathcal{P}_{m}} p_{m} \rightarrow \nabla p \quad \text { weakly-* in } L^{\infty}\left(0, T ; L^{2}(\Omega)^{d}\right) .
\end{aligned}
$$

The zero-average condition in (6) shows that $\int_{\Omega} \Pi_{\mathcal{P}_{m}} p_{m}(\cdot, t)=0$ for all $t \in$ $(0, T)$. Hence, the weak-* convergence of $\Pi_{\mathcal{P}_{m}} p_{m}$ ensures that $\int_{\Omega} p(\cdot, t)=0$ for a.e. $t \in(0, T)$ (test the zero-average condition on $\Pi_{\mathcal{P}_{m}} p_{m}$ with functions $\rho \in L^{\infty}(0, T)$ and pass to the limit).

Consider $\psi(\boldsymbol{x}, t)=\Xi(t) \eta(\boldsymbol{x})$ with $\Xi \in C^{\infty}([0, T])$ and $\eta \in C^{\infty}(\bar{\Omega})$. Define $\Xi_{\delta t_{m}}(t)=\Xi\left(t^{(n+1)}\right)$ on $\left(t^{(n)}, t^{(n+1)}\right)$ for each $n$ and note that $\left(\Xi_{\delta t_{m}}\right)_{m \in \mathbb{N}}$ converges to $\Xi$ uniformly.

By consistency of $\left(\mathcal{P}_{m}\right)_{m \in \mathbb{N}}$, there exists $z_{m} \in \mathcal{P}_{m}$ such that $\Pi_{\mathcal{P}_{m}} z_{m} \rightarrow \eta$ and $\nabla_{\mathcal{P}_{m}} z_{m} \rightarrow \nabla \eta$ strongly in $L^{2}(\Omega)$. Recalling that $A=K / \mu$ satisfies (2c), [16, Lemma C.4] shows that $A\left(\boldsymbol{x}, \widetilde{\Pi}_{\mathcal{C}_{m}} c_{m}\right) \nabla_{\mathcal{P}_{m}} z_{m} \rightarrow A(\boldsymbol{x}, c) \nabla \eta$ strongly in $L^{2}(\Omega \times$ 
$(0, T))^{d}$. Apply the second equation of $(6)$ to $z=\Xi\left(t^{(n+1)}\right) z_{m}$, multiply by $\delta t_{m}^{\left(n+\frac{1}{2}\right)}$, and take the sum over $n=0, \ldots, N_{m}-1$. denoting by $q_{\delta t_{m}}^{ \pm}$the piecewise-constantin-time functions equal to $q_{n}^{ \pm}$on $\left(t^{(n)}, t^{(n+1)}\right)$, we obtain

$$
\begin{aligned}
\int_{0}^{T} \int_{\Omega} A\left(\boldsymbol{x}, \widetilde{\Pi}_{\mathcal{C}_{m}} c_{m}\right) \nabla_{\mathcal{P}_{m}} p_{m} \cdot\left(\Xi_{\delta t_{m}} \nabla_{\mathcal{P}_{m}} z_{m}\right) & \\
& =\int_{0}^{T} \int_{\Omega}\left(q_{\delta t_{m}}^{+}-q_{\delta t_{m}}^{-}\right) \Xi_{\delta t_{m}} \Pi_{\mathcal{P}_{m}} z_{m}
\end{aligned}
$$

By symmetry of $A$, strong convergence of $\widetilde{\Pi}_{\mathcal{C}_{m}} c_{m}$ and of $\nabla_{\mathcal{P}_{m}} z_{m}$, together with the weak convergence of $\nabla_{\mathcal{P}_{m}} p_{m}$, a weak-strong convergence result (see, e.g., [16, Lemma C.3]) shows that the left-hand side of (52) converges to $\int_{0}^{T} \int_{\Omega} A(\boldsymbol{x}, c) \nabla p$. $\Xi \nabla \eta$. Moreover, $q_{\delta t_{m}}^{ \pm} \rightarrow q^{ \pm}$in $L^{1}\left(0, T ; L^{2}(\Omega)\right)$ and thus the right-hand side of (52) converges to $\int_{0}^{T} \int_{\Omega}\left(q^{+}-q^{-}\right) \Xi \eta$. This shows that $p$ satisfies the second equation in (3) when $\psi=\Xi \eta$. By linear combination, this equation is also satisfied for all tensorial functions and, by a density argument, for all smooth functions. Hence, $p$ satisfies (3).

Step 2: strong convergence of $\nabla_{\mathcal{P}_{m}} p_{m}$ and $\mathbf{U}_{\mathcal{P}_{m}}$. Let $z=p_{m}^{(n+1)}$ in (6), multiply by $\delta t_{m}^{\left(n+\frac{1}{2}\right)}$ and take the sum over $n=0, \ldots, N_{m}-1$. By weak convergence of $\Pi_{\mathcal{P}_{m}} p_{m}$ and since $p$ satisfies (3) (which also holds, by density, for $\psi \in L^{1}\left(0, T ; H^{1}(\Omega)\right)$ ),

$$
\begin{aligned}
& \lim _{m \rightarrow \infty} \int_{0}^{T} \int_{\Omega} A\left(\boldsymbol{x}, \widetilde{\Pi}_{\mathcal{C}_{m}} c_{m}\right) \nabla_{\mathcal{P}_{m}} p_{m} \cdot \nabla_{\mathcal{P}_{m}} p_{m} \\
= & \lim _{m \rightarrow \infty} \int_{0}^{T} \int_{\Omega}\left(q_{\delta t_{m}}^{+}-q_{\delta t_{m}}^{-}\right) \Pi_{\mathcal{P}_{m}} p_{m}=\int_{0}^{T} \int_{\Omega}\left(q^{+}-q^{-}\right) p=\int_{0}^{T} \int_{\Omega} A(\boldsymbol{x}, c) \nabla p \cdot \nabla p .
\end{aligned}
$$

This convergence, the weak convergence of $\nabla_{\mathcal{P}_{m}} p_{m}$ and the strong convergence of $A\left(\boldsymbol{x}, \widetilde{\Pi}_{\mathcal{C}_{m}} c_{m}\right) \nabla p$ show that

$$
\begin{aligned}
\int_{0}^{T} & \int_{\Omega} A\left(\boldsymbol{x}, \widetilde{\Pi}_{\mathcal{C}_{m}} c_{m}\right)\left(\nabla_{\mathcal{P}_{m}} p_{m}-\nabla p\right) \cdot\left(\nabla_{\mathcal{P}_{m}} p_{m}-\nabla p\right) \\
= & \int_{0}^{T} \int_{\Omega} A\left(\boldsymbol{x}, \widetilde{\Pi}_{\mathcal{C}_{m}} c_{m}\right) \nabla_{\mathcal{P}_{m}} p_{m} \cdot \nabla_{\mathcal{P}_{m}} p_{m}-\int_{0}^{T} \int_{\Omega} A\left(\boldsymbol{x}, \widetilde{\Pi}_{\mathcal{C}_{m}} c_{m}\right) \nabla_{\mathcal{P}_{m}} p_{m} \cdot \nabla p \\
& -\int_{0}^{T} \int_{\Omega} A\left(\boldsymbol{x}, \widetilde{\Pi}_{\mathcal{C}_{m}} c_{m}\right) \nabla p \cdot\left(\nabla_{\mathcal{P}_{m}} p_{m}-\nabla p\right) \rightarrow 0
\end{aligned}
$$

By coercivity of $A$ (Assumption (2c)), we infer that $\nabla_{\mathcal{P}_{m}} p_{m} \rightarrow \nabla p$ strongly in $L^{2}(\Omega \times(0, T))^{d}$. Moreover, since $\nabla_{\mathcal{P}_{m}} p_{m}$ is bounded in $L^{\infty}\left(0, T ; L^{2}(\Omega)\right)$ (Lemma $6.1)$, this implies that $\nabla_{\mathcal{P}_{m}} p_{m} \rightarrow \nabla p$ strongly in $L^{r}\left(0, T ; L^{2}(\Omega)\right)^{d}$ for any $r \in(1, \infty)$.

Up to a subsequence $\widetilde{\Pi}_{\mathcal{C}_{m}} c_{m} \rightarrow c$ a.e. on $\Omega \times(0, T)$. The properties (2c) of $A$ and the above convergence of $\nabla_{\mathcal{P}_{m}} p_{m}$ show that $\mathbf{U}_{\mathcal{P}_{m}}=-\frac{\mathbf{K}}{\mu\left(\widetilde{\Pi}_{\mathcal{C}_{m}} c_{m}\right)} \nabla_{\mathcal{P}_{m}} p_{m} \rightarrow \mathbf{U}=$ $-\frac{\mathbf{K}}{\mu(c)} \nabla p$ strongly in $L^{r}\left(0, T ; L^{2}(\Omega)\right)^{d}$.

Step 3: strong convergence of $\Pi_{\mathcal{P}_{m}} p_{m}$. Since $p \in L^{2}\left(0, T ; H^{1}(\Omega)\right)$, by [16, Lemma 4.9] we can find $P_{m} \in X_{\mathcal{P}_{m}}^{N_{m}+1}$ such that $\Pi_{\mathcal{P}_{m}} P_{m} \rightarrow p$ and $\nabla_{\mathcal{P}_{m}} P_{m} \rightarrow \nabla p$ strongly in $L^{2}\left(0, T ; L^{2}(\Omega)\right)$. Then, for each $t \in(0, T)$, by definition of the coercivity constant 
$C_{\mathcal{P}_{m}}$,

$\left\|\Pi_{\mathcal{P}_{m}}\left(P_{m}-p_{m}\right)\right\|_{L^{2}(\Omega)}^{2} \leq C_{\mathcal{P}_{m}}^{2}\left(\left\|\nabla_{\mathcal{P}_{m}}\left(P_{m}-p_{m}\right)\right\|_{L^{2}(\Omega)}^{2}+\left|\int_{\Omega} \Pi_{\mathcal{P}_{m}}\left(P_{m}-p_{m}\right)\right|^{2}\right)$.

Integrating from 0 to $T$ and using $\int_{\Omega} p=\int_{\Omega} \Pi_{\mathcal{P}_{m}} p_{m}=0$ yields

$$
\begin{aligned}
& \left\|\Pi_{\mathcal{P}_{m}}\left(P_{m}-p_{m}\right)\right\|_{L^{2}(\Omega \times(0, T))}^{2} \\
& \quad \leq C_{\mathcal{P}_{m}}^{2}\left\|\nabla_{\mathcal{P}_{m}}\left(P_{m}-p_{m}\right)\right\|_{L^{2}(\Omega \times(0, T))^{d}}^{2}+C_{\mathcal{P}_{m}}^{2} \int_{0}^{T}\left|\int_{\Omega}\left(\Pi_{\mathcal{P}_{m}} P_{m}-p\right)\right|^{2} .
\end{aligned}
$$

The first term on the right hand side converges to 0 since both $\nabla_{\mathcal{P}_{m}} P_{m}$ and $\nabla_{\mathcal{P}_{m}} p_{m}$ converge strongly to $\nabla p$ (and $\left(C_{\mathcal{P}_{m}}\right)_{m \in \mathbb{N}}$ is bounded by coercivity of $\left(\mathcal{P}_{m}\right)_{m \in \mathbb{N}}$ ). The second term converges to 0 since $\Pi_{\mathcal{P}_{m}} P_{m}$ converges to $p$ strongly. This shows that $\Pi_{\mathcal{P}_{m}} p_{m}$ also converges strongly to $p$ in this space, and the convergence in $L^{r}\left(0, T ; L^{2}(\Omega)\right)$ follows due to the bound on $\Pi_{\mathcal{P}_{m}} p_{m}$ in Lemma 6.1.

7.3. Convergence of the concentration. The proof of Theorem 3.3 is concluded by showing that $c$ satisfies (4). It has already been established that $c \in$ $L^{2}\left(0, T ; H^{1}(\Omega)\right)$. Lemma 6.2 shows that $\left(1+\left|\mathbf{U}_{\mathcal{P}_{m}}\right|\right)^{1 / 2} \nabla_{\mathcal{C}_{m}} c_{m}$ is bounded in $L^{2}(\Omega \times(0, T))^{d}$ and therefore weakly converges, up to a subsequence, in this space to some $\mathcal{W}$. Since $\mathbf{U}_{\mathcal{P}_{m}}$ converges strongly in $L^{2}(\Omega \times(0, T))^{d}$ and $\nabla_{\mathcal{C}_{m}} c \rightarrow \nabla c$ converges weakly in this space, $\left(1+\left|\mathbf{U}_{\mathcal{P}_{m}}\right|\right)^{1 / 2} \nabla_{\mathcal{C}_{m}} c_{m} \rightarrow(1+|\mathbf{U}|)^{1 / 2} \nabla c$ in the sense of distributions. Hence, $(1+|\mathbf{U}|)^{1 / 2} \nabla c=\mathcal{W} \in L^{2}(\Omega \times(0, T))^{d}$. It remains to prove that the equation in (4) is satisfied.

Take a test function $\varphi(\boldsymbol{x}, t)=\Theta(t) \omega(\boldsymbol{x})$ with $\Theta \in C^{\infty}([0, T))$ and $\omega \in C^{\infty}(\bar{\Omega})$. For $m \in \mathbb{N}$ let $\Theta_{\delta t_{m}}:(0, T) \rightarrow \mathbb{R}$ be such that $\Theta_{\delta t_{m}}=\Theta\left(t^{(n+1)}\right)$ on $\left(t^{(n)}, t^{(n+1)}\right]$ for all $n=0, \ldots, N_{m}-1$ (for legibility, we drop the index $m$ in the time steps $\left.t_{m}^{(k)}\right)$. Using Assumption (A3), define the interpolant $z_{m}:=\mathcal{J}_{\mathcal{C}_{m}} \omega$ of $\omega$. Now, consider $z=\Theta\left(t^{(n+1)}\right) z_{m} \in X_{\mathcal{C}_{m}}$ in (8), so that $v=v_{m}^{(n)}$ is given by $v_{m}^{(n)}\left(\boldsymbol{x}, t^{(n)}\right)=$ $\Theta\left(t^{(n+1)}\right) \Pi_{\mathcal{C}_{m}} z_{m}\left(F_{t^{(n+1)}-t^{(n)}}^{(n+1)}(\boldsymbol{x})\right)$ (here, we make explicit the dependency on the flow $F_{t}^{(n+1)}$ with respect to the time step $n$, but not with respect to $m$ ). Sum the resulting equations over $n=0, \ldots, N_{m}-1$ and recall the definition (35) of $\mathcal{T}_{\mathbf{u}_{\mathcal{P}_{m}}}$. Letting $\hat{q}_{\delta t_{m}}^{ \pm}$be the function equal to $q_{n+1}^{ \pm}$on $\left(t^{(n)}, t^{(n+1)}\right)$ for all $n=0, \ldots, N_{m}-1$, we obtain

$$
\begin{aligned}
& {\left[\sum_{n=0}^{N_{m}-1} \int_{\Omega} \phi \Pi_{\mathcal{C}_{m}} c_{m}^{(n+1)} \Theta\left(t^{(n+1)}\right) \Pi_{\mathcal{C}_{m}} z_{m}-\sum_{n=0}^{N_{m}-1} \int_{\Omega} \phi \Pi_{\mathcal{C}_{m}} c_{m}^{(n)} v_{m}^{(n)}\left(t^{(n)}\right)\right]} \\
& \quad+\int_{0}^{T} \int_{\Omega} \mathbf{D}\left(\boldsymbol{x}, \mathbf{U}_{\mathcal{P}_{m}}\right) \nabla_{\mathcal{C}_{m}} c_{m} \cdot \Theta_{\delta t_{m}}(t) \nabla_{\mathcal{C}_{m}} z_{m} \\
& \quad+\int_{0}^{T} \int_{\Omega}\left[w \widetilde{\Pi}_{\mathcal{C}_{m}} c_{m} \mathcal{T}_{\mathbf{u}_{\mathcal{P}_{m}}}\left[\Theta_{\delta t_{m}}(t) \Pi_{\mathcal{C}_{m}} z_{m}\right] q_{\delta t_{m}}^{-}+(1-w) \Pi_{\mathcal{C}_{m}} c_{m} \Theta_{\delta t_{m}}(t) \Pi_{\mathcal{C}_{m}} z_{m} \hat{q}_{\delta t_{m}}^{-}\right] \\
& \quad=\int_{0}^{T} \int_{\Omega}\left[w \mathcal{T}_{\mathbf{u}_{\mathcal{P}_{m}}}\left[\Theta_{\delta t_{m}}(t) \Pi_{\mathcal{C}_{m}} z_{m}\right] q_{\delta t_{m}}^{+}+(1-w) \hat{q}_{\delta t_{m}}^{+} \Theta_{\delta t_{m}}(t) \Pi_{\mathcal{C}_{m}} z_{m}\right] .
\end{aligned}
$$

Let us write $T_{1}^{(m)}+T_{2}^{(m)}+T_{3}^{(m)}=T_{4}^{(m)}$ this relation.

The limits of the last two terms are the easiest to establish. By the strong convergences of $\Pi_{\mathcal{C}_{m}} c_{m}, \widetilde{\Pi}_{\mathcal{C}_{m}} c_{m}$ and $\Theta_{\delta t_{m}} \Pi_{\mathcal{C}_{m}} z_{m}$ in $L^{2}(\Omega \times(0, T))$, Lemma 5.7 
shows that

$$
T_{3}^{(m)} \rightarrow \int_{0}^{T} \int_{\Omega} c \varphi q^{-} \quad \text { and } \quad T_{4}^{(m)} \rightarrow \int_{0}^{T} \int_{\Omega} q^{+} \varphi .
$$

Let us turn to $T_{2}^{(m)}$. Since $\mathbf{U}_{\mathcal{P}_{m}} \rightarrow \mathbf{U}$ strongly in $L^{2}(\Omega \times(0, T))^{d}$, the growth assumption (2d) on $\mathbf{D}$ ensures that (see, e.g., [19, Lemma A.1])

$$
\mathbf{D}\left(\cdot, \mathbf{U}_{\mathcal{P}_{m}}\right)^{1 / 2} \rightarrow \mathbf{D}(\cdot, \mathbf{U})^{1 / 2} \text { strongly in } L^{4}(\Omega \times(0, T))^{d \times d} .
$$

By Lemma 6.2 the sequence $\mathbf{D}\left(\cdot, \mathbf{U}_{\mathcal{P}_{m}}\right)^{1 / 2} \nabla_{\mathcal{C}_{m}} c_{m}$ is bounded in $L^{2}(\Omega \times(0, T))^{d}$. The weak convergence of $\nabla_{\mathcal{C}_{m}} c_{m}$ in $L^{2}(\Omega \times(0, T))^{d}$ and [19, Lemma A.3] thus show that $\mathbf{D}\left(\cdot, \mathbf{U}_{\mathcal{P}_{m}}\right)^{1 / 2} \nabla_{\mathcal{C}_{m}} c_{m} \rightarrow \mathbf{D}(\cdot, \mathbf{U})^{1 / 2} \nabla c$ weakly in $L^{2}(\Omega \times(0, T))^{d}$. Using (54) and the fact that $\Theta_{\delta t_{m}} \rightarrow \Theta$ uniformly, the strong convergence $\nabla_{\mathcal{C}_{m}} z_{m} \rightarrow \nabla \omega$ in $L^{4}(\Omega)^{d}$ (see $\left.(\mathbf{A 3})\right)$ shows that, as $m \rightarrow \infty$,

$$
\begin{aligned}
T_{2}^{(m)} & =\int_{0}^{T} \int_{\Omega} \mathbf{D}\left(\boldsymbol{x}, \mathbf{U}_{\mathcal{P}_{m}}\right)^{1 / 2} \nabla_{\mathcal{C}_{m}} c_{m} \cdot \mathbf{D}\left(\boldsymbol{x}, \mathbf{U}_{\mathcal{P}_{m}}\right)^{1 / 2} \Theta_{\delta t_{m}}(t) \nabla_{\mathcal{C}_{m}} z_{m} \\
& \rightarrow \int_{0}^{T} \int_{\Omega} \mathbf{D}(\boldsymbol{x}, \mathbf{U})^{1 / 2} \nabla c \cdot \mathbf{D}(\boldsymbol{x}, \mathbf{U})^{1 / 2} \nabla \varphi=\int_{0}^{T} \int_{\Omega} \mathbf{D}(\boldsymbol{x}, \mathbf{U}) \nabla c \cdot \nabla \varphi .
\end{aligned}
$$

We now consider $T_{1}^{(m)}$. Since $\Theta\left(t^{\left(N_{m}\right)}\right)=0$, a change of index in the first sum of $T_{1}^{(m)}$ and recalling the definition of $v_{m}^{(n)}\left(t^{n}\right)$ yield

$$
\begin{aligned}
T_{1}^{(m)}= & \sum_{n=0}^{N_{m}-1} \int_{\Omega} \phi \Pi_{\mathcal{C}_{m}} c_{m}^{(n)} \Theta\left(t^{(n)}\right) \Pi_{\mathcal{C}_{m}} z_{m}-\int_{\Omega} \phi \Pi_{\mathcal{C}_{m}} c_{m}^{(0)} \Theta\left(t^{(0)}\right) \Pi_{\mathcal{C}_{m}} z_{m} \\
& -\sum_{n=0}^{N_{m}-1} \int_{\Omega} \phi \Pi_{\mathcal{C}_{m}} c_{m}^{(n)} \Theta\left(t^{(n+1)}\right) \Pi_{\mathcal{C}_{m}} z_{m}\left(F_{\delta t_{m}^{(n+1 / 2)}}^{(n+1)}(\boldsymbol{x})\right) \\
= & \sum_{n=0}^{N_{m}-1} \int_{\Omega} \phi \Pi_{\mathcal{C}_{m}} c_{m}^{(n)}\left(\Theta\left(t^{(n)}\right)-\Theta\left(t^{(n+1)}\right)\right) \Pi_{\mathcal{C}_{m}} z_{m}-\int_{\Omega} \phi \Pi_{\mathcal{C}_{m}} c_{m}^{(0)} \Theta\left(t^{(0)}\right) \Pi_{\mathcal{C}_{m}} z_{m} \\
& -\sum_{n=0}^{N_{m}-1} \int_{\Omega} \phi \Pi_{\mathcal{C}_{m}} c_{m}^{(n)} \Theta\left(t^{(n+1)}\right)\left(\Pi_{\mathcal{C}_{m}} z_{m}\left(F_{\delta t_{m}^{(n+1 / 2)}}^{(n+1)}(\boldsymbol{x})\right)-\Pi_{\mathcal{C}_{m}} z_{m}\right) \\
= & T_{11}^{(m)}-T_{12}^{(m)}-T_{13}^{(m)} .
\end{aligned}
$$

Since $c_{m}^{(0)}=\mathcal{I}_{\mathcal{C}_{m}} c_{\text {ini }}$, the consistency of $\left(\mathcal{C}_{m}\right)_{m \in \mathbb{N}}$ (see Definition 2.2) ensures that

$$
T_{12}^{(m)} \rightarrow \int_{\Omega} \phi c_{\mathrm{ini}} \Theta(0) \omega=\int_{\Omega} \phi c_{\mathrm{ini}} \varphi(\cdot, 0) .
$$

Since $\Theta\left(t^{(n)}\right)-\Theta\left(t^{(n+1)}\right)=-\int_{t^{(n)}}^{t^{(n+1)}} \Theta^{\prime}$ the strong convergences of $\Pi_{\mathcal{C}_{m}} z_{m}$ and $\widetilde{\Pi}_{\mathcal{C}_{m}} c_{m}$ show that

$$
T_{11}^{(m)}=-\int_{0}^{T} \int_{\Omega} \phi \widetilde{\Pi}_{\mathcal{C}_{m}} c_{m} \Theta^{\prime} \Pi_{\mathcal{C}_{m}} z_{m} \rightarrow-\int_{0}^{T} \int_{\Omega} \phi c \frac{\partial \varphi}{\partial t} .
$$

It remains to analyse $T_{13}^{(m)}$. Let $\zeta_{m}=\Pi_{\mathcal{C}_{m}} z_{m}-\omega$ and write

$$
\Pi_{\mathcal{C}_{m}} z_{m}\left(F_{\delta t^{(n+1 / 2)}}^{(n+1)}\right)-\Pi_{\mathcal{C}_{m}} z_{m}=\left(\omega\left(F_{\delta t^{(n+1 / 2)}}^{(n+1)}\right)-\omega\right)+\zeta_{m}\left(F_{\delta t^{(n+1 / 2)}}^{(n+1)}\right)-\zeta_{m} .
$$


Letting I be the identity map and $\kappa(t)$ be the piecewise-constant function equal to $\delta_{m}^{\left(n+\frac{1}{2}\right)}$ on $\left(t^{(n)}, t^{(n+1)}\right)$, this yields

$$
\begin{aligned}
& T_{13}^{(m)}=\sum_{n=0}^{N_{m}-1} \int_{\Omega} \phi \Pi_{\mathcal{C}_{m}} c_{m}^{(n)} \Theta\left(t^{(n+1)}\right)\left(\omega\left(F_{\delta t_{m}^{(n+1 / 2)}}^{(n+1)}\right)-\omega\right) \\
&+\int_{t^{(1)}}^{T} \int_{\Omega} \frac{\phi}{\kappa(t)} \widetilde{\Pi}_{\mathcal{C}_{m}} c_{m}\left(\mathcal{T}_{\mathbf{u}_{\mathcal{P}_{m}}}-\mathrm{I}\right)\left[\Theta_{\delta_{m}}(t) \zeta_{m}\right] \\
&+\Theta\left(t^{(1)}\right) \int_{\Omega} \phi \Pi_{\mathcal{C}_{m}} c_{m}^{(0)}\left(\zeta_{m}\left(F_{t^{(1)}}^{(1)}\right)-\zeta_{m}\right)
\end{aligned}
$$

We note that, in the last two terms, the case $n>0$ is separated from the case $n=0$, as we do not have any information regarding the boundedness of $\nabla_{\mathcal{C}_{m}} c_{m}^{(0)}$ (which would arise in the estimates after invoking (A2)). For a.e. $\boldsymbol{x} \in \Omega, t \mapsto F_{t}^{(n+1)}(\boldsymbol{x})$ is Lipschitz-continuous and the chain rule therefore yields

$$
\begin{aligned}
\omega\left(F_{\delta t_{m}^{(n+1 / 2)}}^{(n+1)}(\boldsymbol{x})\right)-\omega(\boldsymbol{x}) & =-\int_{t^{(n)}}^{t^{(n+1)}} \partial_{t}\left[\omega\left(F_{t^{(n+1)}-t}^{(n+1)}(\boldsymbol{x})\right)\right] \\
& =\int_{t^{(n)}}^{t^{(n+1)}} \nabla \omega\left(F_{t^{(n+1)}-t}^{(n+1)}(\boldsymbol{x})\right) \cdot \frac{\mathbf{u}_{\mathcal{P}_{m}^{(n+1)}\left(F_{t^{(n+1)}-t}^{(n+1)}(\boldsymbol{x})\right)}}{\phi\left(\left(F_{t^{(n+1)}-t}^{(n+1)}(\boldsymbol{x})\right)\right)} .
\end{aligned}
$$

The operator $\mathcal{T}_{\mathbf{u}_{\mathcal{P}_{m}}}$ does not directly act on the time component in $L^{2}(\Omega \times(0, T))$. Hence, the representation (37) of its dual is also valid in $L^{2}\left(\Omega \times\left(t^{(1)}, T\right)\right)$, and space-independent functions can be taken out of these operators. Using this representation, (58) and recalling the definition (35) of $\widehat{\mathcal{T}}_{\mathbf{u}_{\mathcal{P}_{m}}}$, we obtain

$$
\begin{aligned}
T_{13}^{(m)}= & \int_{0}^{T} \int_{\Omega} \phi \widetilde{\Pi}_{\mathcal{C}_{m}} c_{m} \widehat{\mathcal{T}}_{\mathbf{u}_{\mathcal{P}_{m}}}\left[\Theta_{\delta t_{m}}(t) \nabla \omega \cdot \frac{\mathbf{u}_{\mathcal{P}_{m}}}{\phi}\right] \\
& +\int_{t^{(1)}}^{T} \int_{\Omega} \frac{\phi}{\kappa(t)}\left(\mathcal{T}_{-\mathbf{u}_{\mathcal{P}_{m}}}-\mathrm{I}\right)\left(\widetilde{\Pi}_{\mathcal{C}_{m}} c_{m}\right) \Theta_{\delta t_{m}}(t) \zeta_{m} \\
& +\int_{t^{(1)}}^{T} \int_{\Omega} \frac{R_{m}}{\kappa(t)} \mathcal{T}_{-\mathbf{u}_{\mathcal{P}_{m}}}\left(\phi \widetilde{\Pi}_{\mathcal{C}_{m}} c_{m}\right) \Theta_{\delta t_{m}}(t) \zeta_{m} \\
& +\Theta\left(t^{(1)}\right) \int_{\Omega} \phi \Pi_{\mathcal{C}_{m}} c_{m}^{(0)}\left(\zeta_{m}\left(F_{t^{(1)}}^{(1)}\right)-\zeta_{m}\right)=T_{131}^{(m)}+\cdots+T_{134}^{(m)} .
\end{aligned}
$$

By weak convergence of $\Theta_{\delta t_{m}}(t) \nabla \omega \cdot \mathbf{u}_{\mathcal{P}_{m}} / \phi$ (owing to (b) in (A5)) and strong convergence of $\widetilde{\Pi}_{\mathcal{C}_{m}} c_{m}$, Lemma 5.7 shows that $T_{131}^{(m)} \rightarrow \int_{0}^{T} \int_{\Omega} c \mathbf{u} \cdot \Theta \nabla \omega=\int_{0}^{T} \int_{\Omega} c \mathbf{u}$. $\nabla \varphi$. Using (A2) we have, for $n=1, \ldots, N_{m}-1$,

$$
\frac{\left\|\Pi_{\mathcal{C}_{m}} c_{m}^{(n)}\left(F_{-\delta t_{m}^{(n+1 / 2)}}^{(n+1)}\right)-\Pi_{\mathcal{C}_{m}} c_{m}^{(n)}\right\|_{L^{1}(\Omega)}}{\delta t_{m}^{(n+1 / 2)}} \leq M_{F}\left\|\mathbf{u}_{\mathcal{P}_{m}}^{(n+1)}\right\|_{L^{2}(\Omega)}\left\|\nabla_{\mathcal{C}_{m}} c_{m}^{(n)}\right\|_{L^{2}(\Omega)} .
$$

Hence, invoking (A1),

$$
\begin{aligned}
\left|T_{132}^{(m)}\right| \leq \phi^{*} M_{F} & \left\|\zeta_{m}\right\|_{L^{\infty}(\Omega)}\|\Theta\|_{L^{\infty}(0, T)} \\
& \times \sum_{n=1}^{N_{m}-1} \delta t_{m}^{(n+1 / 2)}\left\|\mathbf{u}_{\mathcal{P}_{m}}^{(n+1)}\right\|_{L^{2}(\Omega)}\left\|\nabla_{\mathcal{C}_{m}} c_{m}^{(n)}\right\|_{L^{2}(\Omega)}
\end{aligned}
$$




$$
\begin{aligned}
& \leq \phi^{*} M_{F}\left\|\zeta_{m}\right\|_{L^{\infty}(\Omega)}\|\Theta\|_{L^{\infty}(0, T)}\left\|\mathbf{u}_{\mathcal{P}_{m}}\right\|_{L^{\infty}\left(0, T ; L^{2}(\Omega)\right)} \\
& \quad \times M_{t} \sum_{n=0}^{N_{m}-2} \delta t_{m}^{(n+1 / 2)}\left\|\nabla_{\mathcal{C}_{m}} c_{m}^{(n+1)}\right\|_{L^{2}(\Omega)} \\
& \leq \phi^{*} M_{F}\left\|\zeta_{m}\right\|_{L^{\infty}(\Omega)}\|\Theta\|_{L^{\infty}(0, T)}\left\|\mathbf{u}_{\mathcal{P}_{m}}\right\|_{L^{\infty}\left(0, T ; L^{2}(\Omega)\right)}\left\|\nabla_{\mathcal{C}_{m}} c_{m}\right\|_{L^{1}\left(0, T ; L^{2}(\Omega)\right)} .
\end{aligned}
$$

Using the bounds on $\mathbf{u}_{\mathcal{P}_{m}}$ and $\nabla_{\mathcal{C}_{m}} c_{m}$ given by (a) in (A5) and Lemmas 6.1 and 6.2, and the convergence $\zeta_{m}=\Pi_{\mathcal{C}_{m}} \mathcal{J}_{\mathcal{C}_{m}} \omega-\omega \rightarrow 0$ in $L^{\infty}(\Omega)$ from $(\mathbf{A 3})$, we infer that $T_{132}^{(m)} \rightarrow 0$. The term $T_{133}^{(m)}$ also converges to 0 , due to the bound on $R_{m}$ in Lemma 5.7 (which cancels out the term $1 / \kappa(t)$ ), the bound (36) and the convergence of $\zeta_{m}$ to 0 in $L^{\infty}(\Omega)$.

Finally, let us study $T_{134}^{(m)}$. Since $\Pi_{\mathcal{C}_{m}} c_{m}^{(0)}=\Pi_{\mathcal{C}_{m}} \mathcal{I}_{\mathcal{C}_{m}} c_{\text {ini }}$ is bounded in $L^{\infty}(\Omega)$ (see Definition 2.2), there is $C$ not depending on $m$ such that $\left|\Theta\left(t^{(1)}\right) \Pi_{\mathcal{C}_{m}} c_{m}^{(0)}\right| \leq C$ a.e. on $\Omega$. Split $\zeta_{m}=\Pi_{\mathcal{C}_{m}} z_{m}-\omega$ and write, using (A2) on $z_{m}$ and Lemma 5.3 on $\omega$

$$
\begin{aligned}
\left|T_{134}^{(m)}\right| & \leq C\left(\left\|\Pi_{\mathcal{C}_{m} z_{m}}\left(F_{t^{(1)}}^{(1)}\right)-\Pi_{\mathcal{C}_{m}} z_{m}\right\|_{L^{1}(\Omega)}+\left\|\omega\left(F_{t^{(1)}}^{(1)}\right)-\omega\right\|_{L^{1}(\Omega)}\right) \\
& \leq C\left\|\mathbf{u}_{\mathcal{P}_{m}}^{(1)}\right\|_{L^{2}(\Omega)}\left|\delta t_{m}^{(1)}\right|\left(M_{F}\left\|\nabla_{\mathcal{C}_{m}} z_{m}\right\|_{L^{2}(\Omega)}+\frac{C_{1}(T)}{\phi_{*}}\|\nabla \omega\|_{L^{2}(\Omega)}\right) .
\end{aligned}
$$

The bounds on $\mathbf{u}_{\mathcal{P}}^{(1)}$ (from (a) in (A5) and Lemma 6.1) and on $\nabla_{\mathcal{C}_{m}} z_{m}$ (from (A3)) then show that $T_{134}^{(m)} \rightarrow 0$.

Hence, $T_{13}^{(m)} \rightarrow \int_{0}^{T} \int_{\Omega} c \mathbf{u} \cdot \nabla \varphi$. Together with (56) and (57), this shows that

$$
T_{1}^{(m)} \rightarrow-\int_{0}^{T} \int_{\Omega} \phi c \frac{\partial \varphi}{\partial t}-\int_{\Omega} \phi c_{\mathrm{ini}} \varphi(\cdot, 0)-\int_{0}^{T} \int_{\Omega} c \mathbf{u} \cdot \nabla \varphi .
$$

Gathering this with (53) and (55), we infer that $c$ satisfies the equation in (4) whenever $\varphi=\Theta \omega$. By linear combination, this equation is also satisfied for all tensorial functions and, by density argument, for all smooth functions. This concludes the proof that $c$ satisfies (4).

\section{Conclusion}

We analysed the convergence of numerical schemes for a coupled elliptic-parabolic system modelling the miscible displacement of a flow by another in a porous medium. The advective terms were discretised by the Eulerian-Lagragian Localised Adjoint Method (ELLAM), and the diffusive terms by the generic framework of the Gradient Discretisation Method (GDM). As a consequence, our analysis applies to a wide range of schemes, given the variety of numerical methods for diffusion problems that fit into the GDM. In particular, our results apply to MFEM-ELLAM of [32] and to the HMM-ELLAM of [12]. The GDM-ELLAM framework also gives an easy way to construct further ELLAM-based schemes, by discretising the diffusion terms using any of the method known to fit into the GDM.

Contrary to previous convergence analysis of schemes involving the ELLAM, the analysis here relies neither on $L^{\infty}$ bounds on the concentration (which, given the anisotropic diffusive terms and generic meshes used in reservoir engineering, would not hold at the discrete level), nor on the smoothness of the data or the solutions (which cannot be established in practical situations, with discontinuous 
data such as the permeability, porosity, etc.). The convergence is established under minimal regularity assumptions on the data, using energy estimates and discrete compactness techniques.

To carry out this analysis, fine properties of the flow of possibly discontinuous Darcy velocities have been established. These properties, as well as some other techniques we develop for the analysis, could certainly prove useful for other characteristic-based discretisations (such as the Modified Method Of Characteristics).

\section{Appendix: GeneriC COMPACTNESS RESUlts}

The following results are particular cases of more general theorems on GDM that can be found in [16].

Lemma 9.1 (Regularity of the limit, space-time problems [16, Lemma 4.7]). Let $p \in(1, \infty)$, and $\left(\left(\mathcal{D}^{T}\right)_{m}\right)_{m \in \mathbb{N}}$ be a coercive and limit-conforming sequence of space-time GDs. For each $m \in \mathbb{N}$, take $u_{m} \in X_{\mathcal{D}_{m}}^{N_{m}+1}$ (identified with a piecewiseconstant function $\left.[0, T] \rightarrow X_{\mathcal{D}_{m}}\right)$ and assume that $\left(\left\|u_{m}\right\|_{L^{p}\left(0, T ; X_{\mathcal{D}_{m}}\right)}\right)_{m \in \mathbb{N}}$ is bounded. Then there exists $u \in L^{p}\left(0, T ; H^{1}(\Omega)\right)$ such that, up to a subsequence as $m \rightarrow \infty$, $\Pi_{\mathcal{D}_{m}} u_{m} \rightarrow u$ and $\nabla_{\mathcal{D}_{m}} u_{m} \rightarrow \nabla u$ weakly in $L^{p}\left(0, T ; L^{2}(\Omega)\right)$. The same property holds with $p=+\infty$, provided that the weak convergences are replaced by weak-* convergences.

Definition 9.2 (Compactly-continuously embedded sequence). Let $\left(X_{m},\|\cdot\|_{X_{m}}\right)_{m \in \mathbb{N}}$ be a sequence of Banach spaces included in $L^{2}(\Omega)$, and $\left(Y_{m},\|\cdot\|_{Y_{m}}\right)_{m \in \mathbb{N}}$ be a sequence of Banach spaces. The sequence $\left(X_{m}, Y_{m}\right)_{m \in \mathbb{N}}$ is compactly-continuously embedded in $L^{2}(\Omega)$ if:

(1) If $u_{m} \in X_{m}$ for all $m \in \mathbb{N}$ and $\left(\left\|u_{m}\right\|_{X_{m}}\right)_{m \in \mathbb{N}}$ is bounded, then $\left(u_{m}\right)_{m \in \mathbb{N}}$ is relatively compact in $L^{2}(\Omega)$.

(2) $X_{m} \subset Y_{m}$ for all $m \in \mathbb{N}$ and for any sequence $\left(u_{m}\right)_{m \in \mathbb{N}}$ such that

(a) $u_{m} \in X_{m}$ for all $m \in \mathbb{N}$ and $\left(\left\|u_{m}\right\|_{X_{m}}\right)_{m \in \mathbb{N}}$ is bounded,

(b) $\left\|u_{m}\right\|_{Y_{m}} \rightarrow 0$ as $m \rightarrow \infty$,

(c) $\left(u_{m}\right)_{m \in \mathbb{N}}$ converges in $L^{2}(\Omega)$, it holds that $u_{m} \rightarrow 0$ in $L^{2}(\Omega)$.

Theorem 9.3 (Discrete Aubin-Simon compactness [16, Theorem 4.17]). Let $\left(X_{m}, Y_{m}\right)_{m \in \mathbb{N}}$ be compactly-continuously embedded in $L^{2}(\Omega), T>0$ and $\left(f_{m}\right)_{m \in \mathbb{N}}$ be a sequence in $L^{2}\left(0, T ; L^{2}(\Omega)\right)$ such that

- For all $m \in N$, there exists $N \in \mathbb{N}^{*}, 0=t^{(0)}<\cdots<t^{(N)}=T$ and $\left(v^{(n)}\right)_{n=0, \ldots, N} \in X_{m}^{N+1}$ such that $f_{m}(t)=v^{(n+1)}$ for all $n=0, \ldots, N-1$ and a.e. $t \in\left(t^{(n)}, t^{(n+1)}\right), f_{m}(t)=v^{(n+1)}$. We then set

$\delta_{m} f_{m}(t)=\frac{v^{(n+1)}-v^{(n)}}{t^{(n+1)}-t^{(n)}}$ for $n=0, \ldots, N-1$ and $t \in\left(t^{(n)}, t^{(n+1)}\right)$.

- The sequence $\left(f_{m}\right)_{m \in \mathbb{N}}$ is bounded in $L^{2}\left(0, T ; L^{2}(\Omega)\right)$.

- The sequence $\left(\left\|f_{m}\right\|_{L^{2}\left(0, T ; X_{m}\right)}\right)_{m \in \mathbb{N}}$ is bounded.

- The sequence $\left(\left\|\delta_{m} f_{m}\right\|_{L^{2}\left(0, T ; Y_{m}\right)}\right)_{m \in \mathbb{N}}$ is bounded.

Then $\left(f_{m}\right)_{m \in \mathbb{N}}$ is relatively compact in $L^{2}\left(0, T ; L^{2}(\Omega)\right)$. 
Acknowledgement: this research was supported by the Australian Government through the Australian Research Council's Discovery Projects funding scheme (project number DP170100605).

\section{REFERENCES}

[1] Y. Alnashri and J. Droniou. Convergence analysis of numerical schemes for non-linear variational inequalities, application to the seepage problem. 2016. Submitted.

[2] T. Arbogast and C. Huang. A fully mass and volume conserving implementation of a characteristic method for transport problems. SIAM Journal on Scientific Computing, 28(6):2001$2022,2006$.

[3] T. Arbogast and W.-H. Wang. Convergence of a fully conservative volume corrected characteristic method for transport problems. SIAM J. Numer. Anal., 48(3):797-823, 2010.

[4] T. Arbogast and W.-H. Wang. Stability, monotonicity, maximum and minimum principles, and implementation of the volume corrected characteristic method. SIAM J. Sci. Comput., 33(4):1549-1573, 2011.

[5] T. Arbogast and M. F. Wheeler. A characteristics-mixed finite element method for advectiondominated transport problems. SIAM J. Numer. Anal., 32(2):404-424, 1995.

[6] S. C. Brenner and L. R. Scott. The mathematical theory of finite element methods, volume 15 of Texts in Applied Mathematics. Springer, New York, third edition, 2008.

[7] F. Brezzi, K. Lipnikov, and M. Shashkov. Convergence of the mimetic finite difference method for diffusion problems on polyhedral meshes. SIAM J. Numer. Anal., 43(5):1872-1896, 2005.

[8] C. Chainais-Hillairet and J. Droniou. Convergence analysis of a mixed finite volume scheme for an elliptic-parabolic system modeling miscible fluid flows in porous media. SIAM J. Numer. Anal., 45(5):2228-2258 (electronic), 2007.

[9] C. Chainais-Hillairet, S. Krell, and A. Mouton. Study of discrete duality finite volume schemes for the Peaceman model. SIAM J. Sci. Comput., 35(6):A2928-A2952, 2013.

[10] C. Chainais-Hillairet, S. Krell, and A. Mouton. Convergence analysis of a DDFV scheme for a system describing miscible fluid flows in porous media. Numer. Methods Partial Differential Equations, 31(3):723-760, 2015.

[11] H. M. Cheng and J. Droniou. Combining the hybrid mimetic mixed method and the eulerian lagrangian localised adjoint method for approximating miscible flows in porous media. In Finite volumes for complex applications. VIII. (Lille, 2017), Springer Proc. Math. Stat. Springer, Cham, 2017.

[12] H. M. Cheng and J. Droniou. An hmm-ellam scheme on generic polygonal meshes for miscible incompressible flows in porous media. 2017.

[13] D. A. Di Pietro and A. Ern. Mathematical aspects of discontinuous Galerkin methods, volume 69 of Mathématiques \& Applications (Berlin) [Mathematics \& Applications]. Springer, Heidelberg, 2012.

[14] J. Droniou. Finite volume schemes for diffusion equations: introduction to and review of modern methods. Math. Models Methods Appl. Sci., 24(8):1575-1619, 2014.

[15] J. Droniou and R. Eymard. A mixed finite volume scheme for anisotropic diffusion problems on any grid. Numer. Math., 105(1):35-71, 2006.

[16] J. Droniou, R. Eymard, T. Gallouët, C. Guichard, and R. Herbin. The gradient discretisation method . Submitted., Nov. 2016.

[17] J. Droniou, R. Eymard, T. Gallouët, and R. Herbin. A unified approach to mimetic finite difference, hybrid finite volume and mixed finite volume methods. Math. Models Methods Appl. Sci., 20(2):265-295, 2010.

[18] J. Droniou, R. Eymard, T. Gallouët, and R. Herbin. Gradient schemes: a generic framework for the discretisation of linear, nonlinear and nonlocal elliptic and parabolic equations. Math. Models Methods Appl. Sci. (M3AS), 23(13):2395-2432, 2013.

[19] J. Droniou and K. S. Talbot. On a miscible displacement model in porous media flow with measure data. SIAM J. Math. Anal., 46(5):3158-3175, 2014.

[20] L. C. Evans and R. F. Gariepy. Measure theory and fine properties of functions. Studies in Advanced Mathematics. CRC Press, Boca Raton, FL, 1992.

[21] R. Eymard, T. Gallouët, and R. Herbin. Finite volume methods. In P. G. Ciarlet and J.L. Lions, editors, Techniques of Scientific Computing, Part III, Handbook of Numerical Analysis, VII, pages 713-1020. North-Holland, Amsterdam, 2000. 
[22] R. Eymard, T. Gallouët, and R. Herbin. Discretization of heterogeneous and anisotropic diffusion problems on general nonconforming meshes SUSHI: a scheme using stabilization and hybrid interfaces. IMA J. Numer. Anal., 30(4):1009-1043, 2010.

[23] R. Eymard, T. Gallouët, and R. Herbin. $\mathcal{R} \mathcal{T}_{k}$ mixed finite elements for some nonlinear problems. Math. Comput. Simulation, 118:186-197, 2015.

[24] X. Feng. On existence and uniqueness results for a coupled system modeling miscible displacement in porous media. J. Math. Anal. Appl., 194(3):883-910, 1995.

[25] V. Girault, J. Li, and B. M. Rivière. Strong convergence of the discontinuous galerkin scheme for the low regularity miscible displacement equations. Numerical Methods for Partial Differential Equations, 33(2):489-513, 2017.

[26] J. Holte. Discrete Gronwall lemma and applications. In MAA-NCS meeting at the University of North Dakota, volume 24, pages 1-7, 2009. http://homepages.gac.edu/ holte/publications/GronwallLemma.pdf.

[27] Y. Kuznetsov and S. Repin. New mixed finite element method on polygonal and polyhedral meshes. Russian Journal of Numerical Analysis and Mathematical Modelling, 18(3), 2003.

[28] K.-A. Lie and B. T. Mallison. Mathematical Models for Oil Reservoir Simulation, pages 850-856. Springer, Berlin, Heidelberg, 2015.

[29] B. Rivière and N. Walkington. Convergence of a discontinuous Galerkin method for the miscible displacement equation under low regularity. SIAM J. Numer. Anal., 49(3):1085$1110,2011$.

[30] T. F. Russell and M. A. Celia. An overview of research on Eulerian-Lagrangian localized adjoint methods (ELLAM). Advances in Water Resources, 25(8):1215 - 1231, 2002.

[31] H. Wang. An optimal-order error estimate for a family of ELLAM-MFEM approximations to porous medium flow. SIAM J. Numer. Anal., 46(4):2133-2152, 2008.

[32] H. Wang, D. Liang, R. E. Ewing, S. L. Lyons, and G. Qin. An approximation to miscible fluid flows in porous media with point sources and sinks by an Eulerian-Lagrangian localized adjoint method and mixed finite element methods. SIAM J. Sci. Comput., 22(2):561-581 (electronic), 2000.

School of Mathematical Sciences, Monash University, Clayton, Victoria 3800, AusTRALIA. HANZ . CHENG@MONASH. EDU

School of Mathematical Sciences, Monash University, Clayton, Victoria 3800, AusTRALIA. JEROME.DRONIOU@MONASH.EDU

School of Mathematics and Statistics, The University of New South Wales, Sydney 2052, Australia. N.Le-Kim@Unsw.edu. au 